\title{
NEUMANN PROBLEM IN ANGULAR REGIONS WITH PERIODIC AND PARABOLIC PERTURBATIONS OF THE BOUNDARY
}

\author{
S. A. NAZAROV
}

\begin{abstract}
We construct and prove asymptotic expansions at infinity for solutions of Neumann problems and matching problems for systems of second order differential equations in regions with corner outlets to infinity. Outside some disc the regions are either periodic or deformed by parabolic inclusions. In addition to logarithmicpolynomial solutions, the asymptotic expansions contain components of the type of boundary layer that either exponentially decay away from the boundary or are localized inside the parabolic inclusions. Operators of the problems become Fredholm operators, and remainders in asymptotic expansions are estimated in scales of function spaces with norms determined by double weight factors and their step distribution. We consider also other types of problems which allow us to apply the developed methods for reduction to a model problem in a sector and for recovery of properties of remainders near perturbed boundary; in particular, we consider the matching problem in regions with irregular points of peak-like inclusion type.
\end{abstract}

\section{Statement of the PRoblem And PReliminary Description of Results}

1.1. Regions. Let $\mathbb{K}=\{x: r>0, \varphi \in(-\alpha, \alpha)\}$ be a sector in the plane $\mathbb{R}^{2}$ with vertex at $\mathcal{O}$, where $x=\left(x_{1}, x_{2}\right)$ and $(r, \varphi)$ are Cartesian and polar coordinates so that $x_{1}=r \cos \varphi, x_{2}=r \sin \varphi$, and $\alpha \in(0, \pi]$ is the half-angle of the sector. We also introduce Cartesian coordinates $y^{ \pm}=\left(y_{1}^{ \pm}, y_{2}^{ \pm}\right)$with the axes $y_{1}^{ \pm}$directed along the sides $(\partial \mathbb{K})^{ \pm}=\{x: r>0, \varphi= \pm \alpha\}$ of the sector, and the axes $y_{2}^{ \pm}$perpendicular to $y_{1}^{ \pm}$and directed inside the sector. All coordinate systems have the origin $\mathcal{O}$. Denote by $H^{ \pm}$the space of smooth periodid 1 functions on the half-line $\mathbb{R}_{+}$, and for $R>0$ let

$$
G_{R}=\left\{x: r>R,|\varphi| \leq \frac{1}{2} \alpha\right\} \cap \bigcup_{ \pm}\left\{x: r>R, \pm \varphi>\frac{1}{2} \alpha, y_{2}^{ \pm}>H^{ \pm}\left(y_{1}^{ \pm}\right)\right\} .
$$

Clearly, in the case of a crack, i.e., for $\alpha=\pi$, we additionally assume that $H^{+}+H^{-}>$ 0 ; then the last two sets on the right-hand side of (1.1) are disjoint. If $\alpha<\pi$ then the disjointness can be obtained by increasing the radius $R$. The boundary of the set (1.1) consists of circular arcs $\mathbb{S}_{R}^{1}$ and periodic curves $\Gamma_{R}^{ \pm}$extending to infinity. By $G$ we mean the region coinciding with (1.1) outside the disc $\mathbb{B}_{R}$ of radius $R$ centered at $\mathcal{O}$ (see Figure 1). For simplicity, we assume that the boundary $\partial G$ is smooth (for generalizations see 3.6 below). The constructed region will be called an angular region with periodic boundary.

Remark 1.1. If $\alpha<\pi$ then without loss of generality we can assume that $H^{ \pm} \geq 0$ and the region (1.1) contains the angular sector $\mathbb{K}_{R}=\{x \in \mathbb{K}: r>R\}$. Indeed, if this is not

2000 Mathematics Subject Classification. Primary 35J25; Secondary 35C20.

The work was financially supported by the Netherlands Organization for Scientific Research (NWO) and the Russian Fund for Scientific Research (RFFI), joint project 047.017.020.

${ }^{1}$ Throughout the paper the period is assumed to be 1 . 


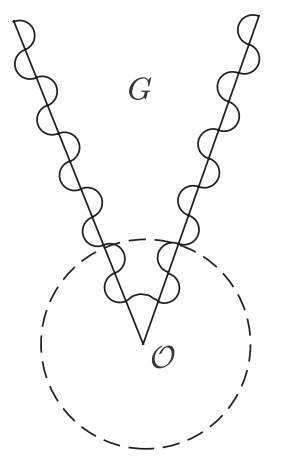

FiguRE 1

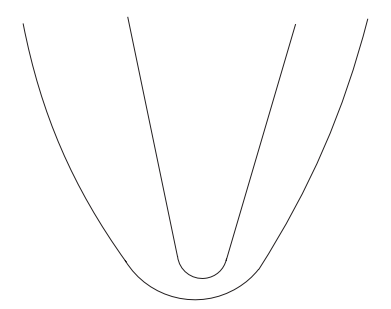

Figure 2

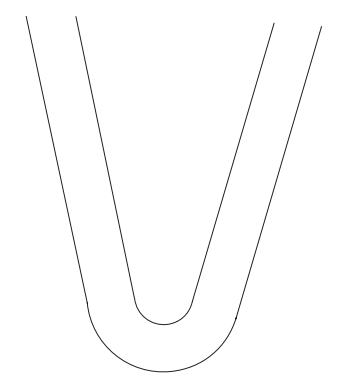

Figure 3

true, then it suffices to shift the origin $\mathcal{O}$ along the bisector inside the sector $\mathbb{K}$ and to make the corresponding changes in notation. This option will be used in the proofs since it make computations much easier. A special case is that of a crack where $\alpha=\pi$ and the shift of the origin is useless. However, the arguments remain true if we replace $\mathbb{K}_{R}$ with the "distorted" sector $\left\{x: r>R,\left|x_{2}\right|>H^{0}\right.$ as $\left.x_{1}<0\right\}$ with $H^{0}>0$; for such a sector reformulations of the results in Section 2 require nothing but obvious modifications.

Let $s \in(0,1)$ and $a_{ \pm} \in \mathbb{R}_{+}$. Formulas

$$
\omega_{R}^{ \pm}=\left\{x: r>R, 0<y_{2}^{ \pm}<a_{ \pm}\left(y_{1}^{ \pm}\right)^{s}\right\}, \quad \omega_{R}^{0}=\mathbb{K}_{R} \backslash\left(\overline{\omega_{R}^{+}} \cup \overline{\omega_{R}^{-}}\right)
$$

determine the angular region $\omega_{R}^{0}$ with parabolic cuts. For $\alpha<\pi$ we set

$$
\Omega_{R}^{ \pm}=\left\{x: r>R, 0>y_{2}^{ \pm}>-a_{ \pm}\left(y_{1}^{ \pm}\right)^{s}\right\}, \quad \Omega_{R}^{0}=\mathbb{K}_{R}
$$

and introduce two regions $\Omega^{1}$ and $\Omega^{0}$ separated by a smooth line $\Gamma^{0}=\partial \Omega^{1} \cap \partial \Omega^{0}$ and coinciding with $\Omega_{R}^{+} \cup \Omega_{R}^{-}$and $\Omega_{R}^{0}$, respectively, outside the disc $\mathbb{B}_{R}$ (see Figure 2). The set $\Omega^{0} \cup \Gamma^{0} \cup \Omega^{1}$ is called a compound angular region with parabolic inclusion. When 
$\alpha=\pi$, we talk about the plane with parabolic inclusion; in this case, $\mathbb{K}=\mathbb{R}^{2} \backslash \mathcal{O}$ is the full angle,

$$
\Omega_{R}^{1}=\left\{x: r>R, x_{1}<0,-a_{-}\left|x_{1}\right|^{s}<x_{2}<a_{+}\left|x_{1}\right|^{s}\right\}, \quad \Omega_{R}^{0}=\mathbb{K}_{R} \backslash \bar{\Omega}_{R}^{1},
$$

and the restriction $a_{ \pm}>0$ is replaced by the condition $a_{-}+a_{+}>0$. The terminology used here reflects the structure of the region at infinity, whereas the geometry of boundaries inside any particular disc is irrelevant. For $s=0$, parabolic sets become half-strips (see Figure 3).

1.2. Neumann boundary problem and matching conditions. Let $\mathcal{D}(\xi)$ be a complex $(m \times k)$-matrix linearly dependent on the variable $\xi=\left(\xi_{1}, \xi_{2}\right)$, where $m \geq k$ and $\mathcal{D}(0)=\mathbb{O}_{m \times k}$ is the zero $(m \times k)$-matrix. Furthermore, let $A$ be a function with values in the space of complex $(m \times m)$-matrices, $A \in C^{\infty}(\bar{G})^{m \times m}$. We assume that the matrix $A(x)$ is Hermitian and positive definite for all $x \in \bar{G}$ and the matrix $\mathcal{D}(\xi)$ has full rank for all $\xi \in \mathbb{R}^{2} \backslash\{0\}$. According to [1, these properties ensure that the matrix $\mathcal{D}(\xi)$ is algebraically complete and the matrix differential operator

$$
L\left(x, \nabla_{x}\right)=\mathcal{D}\left(\nabla_{x}\right)^{*} A(x) \mathcal{D}\left(\nabla_{x}\right)
$$

is formally positive and elliptic. Here $\mathcal{D}\left(\nabla_{x}\right)^{*}={\overline{\mathcal{D}\left(-\nabla_{x}\right)}}^{\top}$ is the differential operator formally conjugate to $\mathcal{D}\left(\nabla_{x}\right)$. Let us emphasize that the algebraic completeness of the operator $\mathcal{D}(\xi)$ implies the polynomial property [2, 3] of the operator $L$ : the corresponding sesquilinear form

$$
a(u, v ; \Xi)=\left(A \mathcal{D}\left(\nabla_{x}\right) u, \mathcal{D}\left(\nabla_{x}\right) v\right)_{\Xi}
$$

degenerates only on vector polynomials in a finite-dimensional algebraic subspace $\mathbf{P}$ of dimension $J$. In (1.6), $\Xi$ is an arbitrary subregion of the plane $\mathbb{R}^{2},(,) \Xi$ is the inner product in the Lebesgue space $L_{2}(\Xi)$ of scalar- or vector-valued functions, as well as its extension by duality between appropriate weight spaces. Finally, the algebraic subspace $\mathbf{P}$ consists of those vector-valued polynomials $p=\left(p_{1}, \ldots, p_{k}\right)^{\top}$ in variables $x=\left(x_{1}, x_{2}\right)$ for which $\mathcal{D}\left(\nabla_{x}\right) p(x)=0$; it is known that this space is finite-dimensional (see [2, 3]). Many facts about boundary value problems used in the sequel follow from this polynomial property (see the survey [3]). Additional requirements on the form (1.6) agree with the following examples.

Example 1.1. 1) Let $k=1, m=2$, let $\mathcal{D}\left(\nabla_{x}\right)=\nabla_{x}$ be a column vector, and $A$ a $(2 \times 2)$-matrix. Then (1.5) is a scalar elliptic operator of the second order and $\mathbf{P}=\mathbb{C}$, $J=1$.

2) Let $k=3, m=6$, and

$$
\mathcal{D}(\xi)^{\top}=\left(\begin{array}{cccccc}
\xi_{1} & 0 & 0 & 0 & 0 & 2^{-1 / 2} \xi_{2} \\
0 & \xi_{2} & 0 & 0 & 0 & 2^{-1 / 2} \xi_{1} \\
0 & 0 & 0 & 2^{-1 / 2} \xi_{2} & 2^{-1 / 2} \xi_{1} & 0
\end{array}\right) .
$$

Then (1.5) is the operator of the system of elasticity theory that describes, in particular, deformations of a two-dimensional compound anisotropic body $\Omega^{0} \cup \Gamma \cup \Omega^{1}$, and $A^{0}$ and $A^{1}$ are the elasticity modulus matrices, which are, of course, real. For isotropic materials,

$$
A^{i}=\left(\begin{array}{cccccc}
\lambda^{i}+2 \mu^{i} & \lambda^{i} & \lambda^{i} & 0 & 0 & 0 \\
\lambda^{i} & \lambda^{i}+2 \mu & \lambda^{i} & 0 & 0 & 0 \\
\lambda^{i} & \lambda^{i} & \lambda^{i}+2 \mu^{i} & 0 & 0 & 0 \\
0 & 0 & 0 & 2 \mu^{i} & 0 & 0 \\
0 & 0 & 0 & 0 & 2 \mu^{i} & 0 \\
0 & 0 & 0 & 0 & 0 & 2 \mu^{i}
\end{array}\right)
$$


where $\lambda^{i} \geq 0$ and $\mu^{i}>0$ are the Lamé coefficients, and the boundary problems and the matching problems that are considered later in the paper break into planar (for the twodimensional Lamé problem) and antiplanar (for the Laplace problem) systems. The role of $\mathbf{P}$ is played by the algebraic subspace of rigid displacements $\left(a_{1}+a_{0} x_{2}, a_{2}-a_{0} x_{1}, a_{3}\right)^{\top}$ of dimension $J=6$.

3) Let $k=4, m=9$,

$$
\mathcal{D}(\xi)^{\top}=\left(\begin{array}{ccccccccc}
\xi_{1} & 0 & 0 & 0 & 0 & 2^{-1 / 2} \xi_{2} & 0 & 0 & 0 \\
0 & \xi_{2} & 0 & 0 & 0 & 2^{-1 / 2} \xi_{1} & 0 & 0 & 0 \\
0 & 0 & 0 & 2^{-1 / 2} \xi_{2} & 2^{-1 / 2} \xi_{1} & 0 & 0 & 0 & 0 \\
0 & 0 & 0 & 0 & 0 & 0 & \xi_{1} & \xi_{2} & 0
\end{array}\right),
$$

let the matrix $A$ be of the form

$$
A=\left(\begin{array}{cc}
A_{11} & A_{12} \\
A_{21} & -A_{22}
\end{array}\right),
$$

where $A_{11}, A_{22}$ are positive definite matrices of size $6 \times 6$ and $3 \times 3$, respectively, and let $A_{12}=A_{21}^{\top}$ be a $(6 \times 3)$-matrix. The corresponding system with the operator (1.5) describes piezoelectric materials. The matrix (1.9) does not satisfy the conditions formulated earlier because of the "wrong" sign in the lower left block; in particular, the operator (1.5) is not formally positive. In [3. Example 1.13] it is shown how a simple change of notation allows one to transform the operator to the form that can be analyzed by the methods used in the present paper.

In the region $G$ we consider the Neumann problem

$$
L\left(x, \nabla_{x}\right) u(x)=f(x), x \in G ; \quad N\left(x, \nabla_{x}\right) u(x)=g(x), x \in \partial G,
$$

where $u=\left(u_{1}, \ldots, u_{k}\right)^{\top}, f, g$ are height $k$ columns of functions, and the operator of boundary conditions

$$
N\left(x, \nabla_{x}\right)=\overline{\mathcal{D}(\nu(x))}^{\top} A(x) \mathcal{D}\left(\nabla_{x}\right)
$$

is taken from the Green formula

$$
(L u, v)_{G}+(N u, v)_{\partial G}=a(u, v ; G):=\left(A \mathcal{D}\left(\nabla_{x}\right) u, \mathcal{D}\left(\nabla_{x}\right) v\right)_{G} .
$$

The vector $\nu$ on the right-hand side of (1.11) is the outward normal vector of the surface $\partial G$.

For the compound region $\Omega^{0} \cup \Gamma^{0} \cup \Omega^{1}$, the matrix $A$ has jumps on the curve $\Gamma^{0}$; on the other hand, the restriction $A^{i}$ of $A$ to the subregion $\Omega^{i}$ is smooth up to the boundary $\partial \Omega^{i}, i=0,1$. Therefore, the Neumann problem corresponding to (1.10) includes the matching conditions, namely

$$
\begin{gathered}
L^{i}\left(x, \nabla_{x}\right) u^{i}(x)=f^{i}(x), x \in \Omega^{i}, \quad N^{1}\left(x, \nabla_{x}\right) u^{1}(x)=g(x), x \in \partial \Omega^{1} \backslash \Gamma^{0}, \\
u^{0}(x)=u^{1}(x), \quad N^{0}\left(x, \nabla_{x}\right) u^{0}(x)=N^{1}\left(x, \nabla_{x}\right) u^{1}(x), x \in \Gamma^{0} .
\end{gathered}
$$

The operators $L^{i}$ and $N^{i}$ are determined by formulas (1.5) and (1.11), respectively, with the matrix $A$ replaced by $A^{i}$, and $\nu$ always denotes the outward normal to the surface $\partial \Omega^{1}$. By $u^{i}$ and $f^{i}$ we denote the restrictions of the functions $u$ and $f$ to $\Omega^{i}, i=0,1$.

The stated problems turn out to be formally selfadjoint. For example, since the form (1.6) is symmetric, (1.12) implies the "full" Green formula

$$
(L u, v)_{G}+(N u, v)_{G}=(u, L v)_{G}+(u, N v)_{\partial G},
$$

which serves the boundary problem (1.10) and holds, e.g., for smooth compactly supported vector-valued functions $u, v \in C_{c}^{\infty}(\bar{G})^{k}$. The Green formula for problem (1.13) can be obtained from (1.14) by obvious changes of the sets $G, \partial G$, and the corresponding 


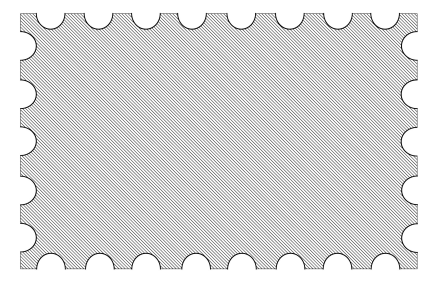

FiguRE 4

differential operators; the test functions $u^{i}, v^{i} \in C_{c}^{\infty}\left(\overline{\Omega^{i}}\right)^{k}, i=0,1$, should additionally satisfy the matching conditions in the second line of (1.13).

Next we assume that in the problem (1.10) we have

$$
A(x)=\mathbf{A} \quad \text { for } \quad x \in G, r>R,
$$

and in the problem (1.13),

$$
A^{0}(x)=\mathbf{A} \quad \text { for } \quad x \in \Omega_{R}^{0}, \quad A^{1}(x)=\mathbf{A}^{ \pm} \quad \text { for } \quad x \in \Omega_{R}^{ \pm},
$$

where $\mathbf{A}$ and $\mathbf{A}^{ \pm}$are constant matrices. Here one could only assume that our matrixvalued functions stabilize at infinity with polynomial rate or to allow for the dependence of $\mathbf{A}$ and $\mathbf{A}^{ \pm}$on the variables $\varphi$ and $\zeta^{ \pm}=\left|y_{1}^{ \pm}\right|^{-\gamma} y_{2}^{ \pm}$, respectively. However, once the main difficulties are resolved, such generalizations become clear (see 3.6 below) and we skip them to simplify the exposition.

1.3. Motivations. In the process of averaging boundary problems in regions $\Omega_{\varepsilon}$ with rapidly oscillating boundaries (see Figure 4) one should take into account the boundary layer phenomenon, which is described using solutions of problems in a half-space or in a half-cylinder with a skew base; this phenomenon allows one to formulate correct boundary conditions on the limiting ("straightened") boundary $\partial \Omega_{0}$. Although the asymptotic analysis was performed only for some simplest boundary problems for the Poisson equation (see [4, 5, 6, 7]), generalizations of these procedures to general boundary problems for elliptic systems with polynomial property (see [2, 3]) do not posepresent any serious difficulties if the boundary $\partial \Omega_{0}$ is smooth. If the boundary is only piecewise smooth, the known algorithms, which require additional smoothness to establish asymptotics, fail even for the Poisson equation because the smoothness of the limiting problem in $\Omega_{0}$ decreases at corner points, on edges, and in other irregular points of the boundary. To improve the situation, one should include boundary layers of a different kind; such boundary layers are formed from solutions of problems in regions with corners and with periodic boundaries, and, contrary to boundary layers mentioned earlier, are characterized by power rather than exponential decrease at infinity. This last property makes the construction of asymptotics much more difficult. However, the required mathematical techniques, namely various methods of matching asymptotic expansions, which are essentially equivalent (but complement each other in details), were developed already in the 1970s and 1980s (see, e.g., books [8] and [9]), and the actual difficulty lies only in the study of limit problems in regions with unusual reach to infinity.

Due to oscillations of the boundary near the point at infinity, classical results of the theory of boundary problems in regions with conical or corner points (see, e.g., key papers [10, 11, 12] and books [13, 14]) are not directly applicable to the boundary problem (1.10). In 15 the author considered a similar Dirichlet problem

$$
L\left(x, \nabla_{x}\right) u(x)=f(x), x \in G ; \quad u(x)=g(x), x \in \partial G,
$$




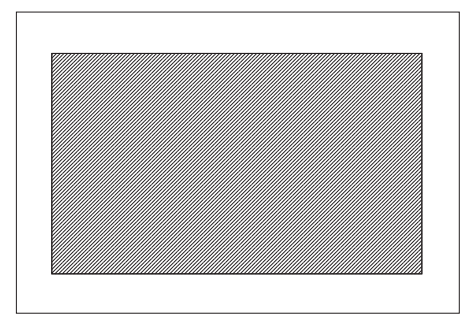

FIGURE 5

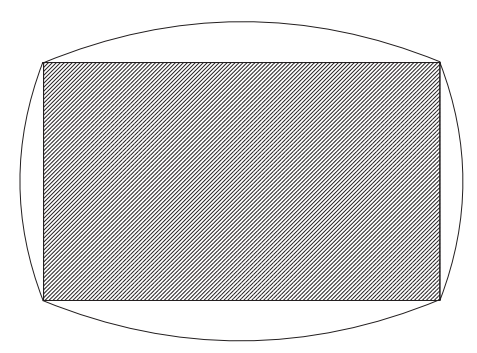

Figure 6

which, after being supplied with special weight spaces, was reduced to the model problem in the sector $\mathbb{K}$. This made it possible to give an asymptotic representation for the solution "modified" near the periodic curves $\Gamma_{R}^{ \pm}$and then, by constructing boundary layers near these curves, to extend the asymptotic formula to the entire region (1.1). The reduction methods developed in [15] were tied with the Dirichlet boundary conditions because they were using, in an essential way, the natural property of solutions to decrease near the boundary. In general, the study of the Dirichlet problem is often easier than the study of other boundary problems (on averaging in regions with corners see [16]-[20]; in the last paper, due to the Dirichlet conditions at the boundary of a perforated region, the corner boundary layer decreases even exponentially).

In the present paper we obtain asymptotic expansions at infinity for solutions of the Neumann problem (1.10) and the matching problem (1.13). The origin of the second problem is also connected with asymptotic analysis, namely, the study of so-called regions with thin boundary layer. Let us discuss a couple of examples.

Example 1.2. 1) Let $Q_{\varepsilon}$ be a rectangle $\left\{\eta=\left(\eta_{1}, \eta_{2}\right):\left|\eta_{i}\right|<l_{i}+\varepsilon, i=1,2\right\}$, on which the scalar differential equation

$$
\nabla_{\eta}^{\top} A(\varepsilon, \eta) \nabla_{\eta} w(\varepsilon, \eta)=f(\eta), \quad \eta \in Q_{\varepsilon} \backslash \partial Q_{0},
$$

is given, with Dirichlet boundary conditions (for simplicity) and natural matching conditions on the polygonal line $\partial Q_{0}$ (see Figure 5 and Example 1.1, 1)). Here $A$ is a piecewise constant function, $A=A_{0}>0$ inside and $A=A_{1}>0$ outside the rectangle $Q_{0}$, and $\varepsilon>0$ is a small parameter. One can easily see that the coordinate change $\eta \mapsto x=\varepsilon^{-1}(\eta-P)$ (where $P$ is one of the vertices of the rectangle $Q_{0}$, say $P=\left(l_{1}, l_{2}\right)$ ) and subsequent formal passage to the limit $\varepsilon=0$ transform equation (1.18) to the equation in (1.13) with matching conditions $(1.13)_{2}$, and transform $Q_{\varepsilon}$ to the compound region with a half-strip inclusion. In other words, in formula (1.3) we have $a_{ \pm}=1$ and $s=0$.

2) Now let the layer have variable cross-section which becomes thinner when approaching to corners (it could have been originally designed this way or could have worn out in the process of using the equipment; such a layer is called "imperfect"; see Figure @). For 


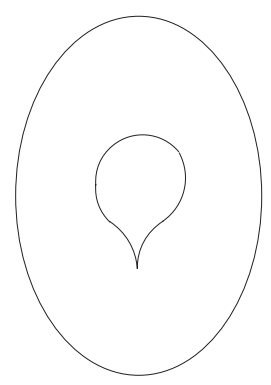

FiguRE 7

example, suppose that, over the upper side of the rectangle, the strip $Q_{\varepsilon} \backslash \bar{Q}_{0}$ has the form

$$
\left\{\eta:\left|\eta_{1}\right|<l_{1}, \eta_{2} \in\left(l_{2}, l_{2}+\varepsilon \mathcal{H}\left(\eta_{1}\right)\right)\right\},
$$

where $\mathcal{H}$ is a smooth positive function on the interval $\left(-l_{1}, l_{1}\right)$ that admits a representation

$$
\mathcal{H}\left(\eta_{1}\right)=\left(b_{1}-\eta_{1}\right)^{s}\left(a_{+}+\mathcal{H}_{+}\left(\eta_{1}\right)\right), \quad \mathcal{H}_{+} \in C^{\infty}\left[0, l_{1}\right], \quad \mathcal{H}_{+}\left(l_{1}\right)=0, \quad s \in(0,1) .
$$

It is not difficult to see that the change of coordinates leading to a region with parabolic inclusion (1.3) is

$$
\eta \mapsto x=\varepsilon^{\tau}(\eta-P), \quad \tau=(1-s)^{-1} .
$$

Regarding Example 1.2, 1), we mention [21]. No strict asymptotic analysis of rounded or worn-out coverings described in Example 1.2, 2) has been performed.

Another application of the results that will be obtained for the problem (1.13) is related to the observation that will be discussed in details in 6.5. The point is that the inversion $x \mapsto \eta=|x|^{-2} x$ transforms the plane with a periodic inclusion (see definition (1.4)) into a compound region $\omega$ with peak inclusion (see Figure 7) for which

$$
\omega_{d}^{1}=\left\{\eta:|\eta|<d, \eta_{1}<0,-b_{-}\left(\eta_{1}\right)\left|\eta_{1}\right|^{z}<\eta_{2}<b_{+}\left(\eta_{1}\right)\left|\eta_{1}\right|^{z}\right\}, \quad \omega_{d}^{0}=\mathbb{B}_{d} \backslash \overline{\omega_{d}^{1}} .
$$

Here $d=1 / R, z=2-s>1$, and $b_{ \pm}$are smooth functions such that $b_{ \pm}(0)=a_{ \pm}$.

The system of equations of elasticity theory (Example 1.1(2)), the ideal mesh conditions $(1.13)_{2}$, and Neumann boundary conditions with exterior forces $g$ on the right-hand side form a known problem of failure theory. The book 22 mentions the construction of asymptotic expansions of solutions near such geometric irregularities as an unsolved problem. For a long time, in the literature on mechanics, it was claimed that elastic fields are singular at the vertex $\mathcal{O}$ of the inclusion $\omega_{d}^{1}$, and moreover, "computations" of the corresponding stress coefficients were given. In the papers [23, 24] it was shown (on the physical level of rigor) that the stresses remain bounded at the point $\mathcal{O}$ and the failure process may be related only to the concentration of stresses. Of course, formal asymptotic Ansatzen require justification, and the corresponding explanations are given in 5.6. Let us emphasize that similar Ansatzen 25] for three-dimensional peak inclusions and holes were justified in [26. Earlier studies were not suitable for this purpose because some properties of model problems postulated in 27] (see also 14]) disappear in the case of three-dimensional system of elasticity theory.

1.4. Difference between functional statements of the Dirichlet and the Neumann problems. In [15] it was shown that the natural classes where the operator of the Dirichlet problem (1.17) acquires the Fredholm property and the estimates for remainders in asymptotic expansions of solutions become sharp are the spaces $\mathfrak{V}_{\beta, \gamma}^{l}(G)$ obtained 
by completing the algebraic subspace $C_{c}^{\infty}(\bar{G})$ (of infinitely differentiable functions with compact support) with respect of the weight norm

$$
\left\|z ; \mathfrak{V}_{\beta, \gamma}^{l}(G)\right\|=\left(\sum_{p=0}^{l}\left\|(1+r)^{\beta-\gamma} \rho^{\gamma-l+p} \nabla_{x}^{p} z ; L_{2}(G)\right\|^{2}\right)^{1 / 2} .
$$

Here $l \in \mathbb{N}_{0}:=\{0,1,2, \ldots\}, \beta, \gamma \in \mathbb{R}$, and the weight $\rho$ is defined by the formula

$$
\rho(x)=1+(1+r)|\alpha-| \varphi|| .
$$

Very important are the following properties of this weight: first, in each cut-off infinite sector (later just a sector) $\mathbb{K}_{R}\left(\alpha_{1}\right):=\left\{x: r \geq R,|\varphi| \leq \alpha_{1}\right\}$ with $\alpha_{1} \in(0, \alpha)$, the weight factor (1.21) is equivalent to $1+r$, i.e.,

$$
0<c\left(\alpha_{1}\right) \leq(1+r)^{-1} \rho(x) \leq C\left(\alpha_{1}\right), \quad x \in \mathbb{K}_{R}\left(\alpha_{1}\right) ;
$$

second, in any bounded subregion $G \cap \mathbb{B}_{d}$ and in any boundary strip $\mathcal{V}_{d}=\{x \in G$ : $\operatorname{dist}(x, \partial G) \leq d\}$ we have $1 \leq \rho(x) \leq c(d)$, i.e., the weight is bounded and separated from zero. The latter property determines the main difference of norms $(1.20)$ from the Kondrat'ev norms [10] defined by the formula

$$
\left\|z ; V_{\beta}^{l}(\mathbb{K})\right\|=\left(\sum_{p=0}^{l}\left\|r^{\beta-l+p} \nabla_{x}^{p} z ; L_{2}(\mathbb{K})\right\|^{2}\right)^{1 / 2},
$$

which are normally used in regions with conic and corner points and exits to infinity. In particular, the properties of trace spaces change as well: the function $Z$ and its derivatives $\partial_{s}^{p} Z, p=1, \ldots, l-1$, acquire a common weight factor $(1+r)^{\beta-\gamma}$ in the trace norm

$$
\left\|z ; \mathfrak{V}_{\beta, \gamma}^{l-1 / 2}(\partial G)\right\|=\inf \left\{\left\|Z ; \mathfrak{V}_{\beta, \gamma}^{l}(G)\right\| \mid Z=z \text { on } \partial G\right\},
$$

whereas similar weight factors in the Kondrat'ev trace norms

$$
\left\|z ; V_{\beta}^{l-1 / 2}(\partial \mathbb{K})\right\|=\inf \left\{\left\|Z ; V_{\beta}^{l}(\mathbb{K})\right\| \mid Z=z \text { on } \partial \mathbb{K}\right\},
$$

are $r^{\beta-l+1 / 2}$ and $r^{\beta-l+p+1 / 2}$, respectively.

Lemma 1.1. 1) For $l \in \mathbb{N}=\{1,2,3, \ldots\}$ the norm (1.23) is equivalent to the norm

$$
\left\|\left(1+r^{2}\right)^{(\beta-\gamma) / 2} z ; H^{l-1 / 2}(\partial G)\right\|,
$$

where $H^{l-1 / 2}(\partial G)$ is the Sobolev-Slobodetskii space.

2) For each right-hand side $g \in \mathfrak{V}_{\beta, \gamma}^{l-1 / 2}(\partial G)^{k}$ there exists a vector-valued function $v \in \mathfrak{V}_{\beta, \gamma}^{l+1}(G)^{k}$ that is supported in the closure $\overline{\mathcal{V}}_{d}$ of the boundary strip and satisfies the boundary condition

$$
N\left(x, \nabla_{x}\right) v(x)=g(x), \quad x \in \partial G,
$$

and the estimate

$$
\left\|v ; \mathfrak{V}_{\beta, \gamma}^{l+1}(G)\right\| \leq c\left\|g ; \mathfrak{V}_{\beta, \gamma}^{l-1 / 2}(\partial G)\right\| .
$$

Comments to the proof are given in Remark 4.1

Let us note that the spaces $\mathfrak{V}_{\beta}^{l}(G)$ and $V_{\beta}^{l}(\mathbb{K})$ consist of functions that belong to the classes $H_{\text {loc }}^{l}(\bar{G})$ and $H_{\text {loc }}^{l}(\overline{\mathbb{K}} \backslash \mathcal{O})$, respectively, i.e., the weight factors determine the behavior of elements of these spaces only at infinity (in the second case, at the vertex of the corner as well).

In [15] it is established that under certain restrictions to weight indices $\beta$ and $\gamma$, the Dirichlet problem operator (1.17), viewed as a map

$$
\mathfrak{V}_{\beta, \gamma}^{l+1}(G)^{k} \rightarrow \mathfrak{V}_{\beta, \gamma}^{l-1}(G)^{k} \times \mathfrak{V}_{\beta, \gamma}^{l-1 / 2}(\partial G)^{k},
$$


turns out to be a Fredholm operator, and for small $|\beta-l|$ it is an isomorphism. The fact that the right-hand side $g \in \mathfrak{V}_{\beta, \gamma}^{l-1 / 2}(\partial G)^{k}$ of the boundary condition (1.25) can be compensated by a vector-valued function in the space $\mathfrak{V}_{\beta, \gamma}^{l+1}(G)^{k}$ (see Lemma 1.1(2)) does not necessarily mean that the Neumann problem (1.10) is normally solvable in this class. Indeed, under the condition

$$
|\gamma-l|<\frac{1}{2}
$$

which is necessary for the operator (1.27) to be Fredholm, the function $\varphi \mapsto|\alpha-| \varphi||^{\gamma-l-1}$ extracted, according to definitions (1.21) and (1.20), from the weight factor $u=\nabla_{x}^{0} u$ in the norm $\left\|u ; \mathfrak{V}_{\beta, \gamma}^{l+1}(G)\right\|$, is not square-integrable on the arc $\Upsilon=(-\alpha, \alpha)$. Therefore, a solution $u$ of the Dirichlet problem decreases (in some sense) when it approaches the sides of an angle, whereas this behavior is not typical for the Neumann problem even if the boundary conditions (1.25) are homogeneous. In other words, one should not expect that the operator of the problem (1.10) viewed as a map similar to (1.27) has good properties.

As in 26, 28, 29, a proper domain for the operator of the Neumann problem is a space with step weight norm. Namely, for $l \in \mathbb{N}_{0}$ and $\beta, \gamma \in \mathbb{R}$ the space $\mathfrak{V}_{\beta, \gamma}^{l, 0}(G)^{k}$ is defined as the completion of the algebraic subspace $C_{c}^{\infty}(\bar{G})^{k}$ with respect to the norm

$$
\left\|z ; \mathfrak{V}_{\beta, \gamma}^{l, 0}(G)\right\|=\left(\sum_{p=0}^{l+1}\left\|(1+r)^{\beta-\gamma-\delta_{p, 0}} \rho^{\gamma-l+p+\delta_{p, 0}} \nabla_{x}^{p} z ; b L_{2}(G)\right\|^{2}\right)^{1 / 2} .
$$

Here $\delta_{p, q}$ is the Kronecker symbol. In the norm (1.20) the graph of the exponent $\rho$ as a function of the differentiation order $p$ is a tilted line, but for $\kappa>0$ a similar graph in the norm (1.29) has a typical horizontal piece (step) at the level $p=0$. This is why the latter weight norms are called step weight norms.

Weight factors in the norm (1.29) are equal to $(1+r)^{\beta-l+p}$ inside the sector $\mathbb{K}_{R}\left(\alpha_{1}\right)$ with half-angle $\alpha_{1}<\alpha$ and $(1+r)^{\beta-\gamma-\delta_{p, 0}}$ in the strip $\mathcal{V}_{d}$. However, later we will not need the trace space for $\mathfrak{V}_{\beta, \gamma}^{l, 0}(G)$, since, by (1.11), the expression $N u$ is a linear combination of the first derivatives of the vector-valued function $u$. The operator (1.5) does not have a free term either. Therefore, the map

$$
\{L, N\}: \mathfrak{V}_{\beta, \gamma}^{l+1,0}(G)^{k} \rightarrow \mathfrak{V}_{\beta, \gamma}^{l-1}(G)^{k} \times \mathfrak{V}_{\beta, \gamma}^{l-1 / 2}(G)^{k}
$$

is continuous for all $l \in \mathbb{N}$ and $\beta, \gamma \in \mathbb{R}$. In Section 4 we will show that the operator (1.30) acquires good properties under the condition

$$
\gamma-l \in(-1 / 2,0),
$$

which is more restrictive than (1.28).

1.5. The structure of the paper. In Section 2 we study the Neumann problem for a differential operator with constant coefficients in a sector. Complete information about this problem, of course, is available in the scale of Kondrat'ev spaces. However, to study boundary value problems in a region $G$ with periodic boundary, we need a different scale of function spaces such that the norms in these spaces have two types of weight factors, $r$ and $\alpha-|\varphi|$, and the spaces contain functions with singularities on the sides of the sector. Therefore, the study of this model problem must be performed completely: the spectral pencil on an arc in special weight classes is analyzed, then the bounds and asymptotic formulas for solutions are deduced, and the necessary and sufficient conditions for the operator of the problem to be an isomorphism are obtained. In addition to asymptotic expansions at infinity and near the vertex $\mathcal{O}$ we obtain asymptotic representations of solutions near the sides $(\partial \mathbb{K})^{ \pm}$of the sector $\mathbb{K}$. These representations involve a certain 
extension operator $\mathfrak{C}$ and play an important role in Section 4 when proving the main theorem of this paper.

In Section 3 we describe a formal procedure for constructing asymptotic expansions. In the case of a periodic boundary, these constructions are more difficult than in the case of parabolic inclusions: in addition to polynomial-logarithmic solutions of a model problem in a sector we also encounter boundary layers, i.e., functions that exponentially decrease in directions perpendicular to the sides of the sector. In other words, the expansion of a solution of the problem (1.10) becomes multiscale and contains asymptotic blocks (3.17). Boundary layers are described using solutions of a problem in a half-infinite strip with curved base, and the conditions of their decay at infinity provide boundary data on $(\partial \mathbb{K})^{ \pm}$in the model problem (see 2.5 and 3.1 ).

The matching problem (1.13) does not involve boundary layers, and, as shown in 3.3, the boundary conditions on $\partial \mathbb{K} \backslash \mathcal{O}$ in the model problem are obtained by analyzing a system of ordinary differential equations on the segment that is a section of the parabolic inclusion.

Section 4 contains the technically most difficult part of the paper. First, using appropriate cut-off functions and increasing the weight index $\gamma$ (an important trick borrowed from [15]), we reduce problem (1.10) in the region $G$ with periodic boundary to the model problem in a sector $\mathbb{K}$. Then, using results of Section 2 , we write an asymptotic formula for the restriction of a solution to the region $\omega_{R}^{0}$ with parabolic cuts; in doing so, polynomial-logarithmic solutions are replaced with asymptotic blocks. Finally, the estimate of the remainder in the asymptotic formula is extended from $\omega_{R}^{0}$ to the entire region $G$. In doing this, we use two tricks: we increase weight factors in the norm of a solution of a mixed boundary value problem and smoothen the operator $\mathfrak{C}$ in 2.4. Simultaneously, we prove the Korn weight inequality (4.45).

In Subsection 5.1 we prove the existence of generalized and classical solutions of the problem (1.10) provided the following orthogonality condition holds:

$$
\int_{G} f(x) d x+\int_{\partial G} g(x) d s_{x}=0 \in \mathbb{C}^{k} .
$$

In the proof, we assume Korn inequality (5.3) with certain weight factors in a sector; this assumption is justified, e.g., in the case of problems of mathematical physics mentioned in Example 1.1 (see also Example 5.1 below). In the case where condition (1.32) is not satisfied, the problem still has so-called "almost energy" solutions, which grow logarithmically at infinity (Proposition 5.2). Finally, in Subsection 5.3 we discuss "non-energy" solutions of the homogeneous problem (1.10). They grow exponentially at infinity and provide integral representations of coefficients in decompositions of decreasing solutions.

Procedures developed in Sections 2-4 can also be used, of course, for problems other than those in sectors with periodic boundaries. In Section 6 we discuss already mentioned problems of matching in regions described by formulas (1.3), (1.4), or (1.19), as well as other possible generalizations.

1.6. Notation. If a group of formulas has a tag $(m . n)$, then $(m . n)_{q}$ denotes the $q$ th line formula in this group; for example, $(1.13)_{2}$ is the pair of matching conditions in problem $(1.13)$. If $\mathfrak{B}$ is a function space, then $\mathfrak{B}^{k}$ is the space of height $k$ columns of elements of $\mathfrak{B}$; however, the index $k$ is omitted in the corresponding norm $\|\cdot ; \mathfrak{B}\|$.

\section{Model PRoBlems}

2.1. Model problem in a sector. We consider the Neumann problem

$$
\mathbf{L}\left(\nabla_{x}\right) u(x)=f(x), \quad x \in \mathbb{K} ; \quad \mathbf{N}^{ \pm}\left(\nabla_{x}\right) u(x)=g(x), \quad x \in(\partial \mathbb{K})^{ \pm},
$$


where the differential operators are of the form

$$
\mathbf{L}\left(\nabla_{x}\right)=\mathcal{D}\left(\nabla_{x}\right)^{*} \mathbf{A} \mathcal{D}\left(\nabla_{x}\right), \quad \mathbf{N}^{ \pm}\left(\nabla_{x}\right)={\overline{\mathcal{D}\left(\nu^{ \pm}\right)}}^{\top} \mathbf{A} \mathcal{D}\left(\nabla_{x}\right)
$$

(cf. (1.5) and (1.10), the matrix $\mathbf{A}$ is taken from relations (1.15) or (1.16), and $\nu^{ \pm}$are outward unit normals to the sides $(\partial \mathbb{K})^{ \pm}$of the sector $\mathbb{K}$. In polar coordinates $(r, \varphi)$ the operators (2.2) take the form

$$
\begin{aligned}
\mathbf{L}\left(\nabla_{x}\right) & =r^{-2} \mathfrak{L}\left(\varphi, \partial_{\varphi}, r \partial_{r}\right)=r^{-2} \mathfrak{D}\left(\varphi, \partial_{\varphi} r \partial_{r}-1\right)^{*} \mathbf{A} \mathfrak{D}\left(\varphi, \partial_{\varphi}, r \partial_{r}\right), \\
\mathbf{N}^{ \pm}\left(\nabla_{x}\right) & =r^{-1} \mathfrak{N}^{ \pm}\left(\partial_{\varphi}, r \partial_{r}\right)=r^{-1}{\overline{D\left(\nu^{ \pm}\right)}}^{\top} \mathbf{A} \mathfrak{D}\left(\varphi, \partial_{\varphi}, r \partial_{r}\right) .
\end{aligned}
$$

Here $r^{-1} \mathfrak{D}\left(\varphi, \partial_{\varphi}, r \partial_{r}\right)$ is the matrix differential operator $\mathcal{D}\left(\nabla_{x}\right)$ written in polar coordinates.

According to the theory of elliptic boundary value problems in regions with conic (corner) points (see the key papers [10, 11, 12, books [13, 14, and other publications) the solvability of problem (2.1) and the properties of solutions are determined by the spectrum of the problem on the arc

$$
\mathfrak{L}\left(\varphi, \partial_{\varphi}, \lambda\right) U(\varphi)=0, \quad \varphi \in \Upsilon=(-\alpha, \alpha) ; \quad \mathfrak{N}^{ \pm}\left(\partial_{\varphi}, \lambda\right) U( \pm \alpha)=0 .
$$

The operators in equations (2.4) are obtained from the operators (2.3) by replacing the differential expression $r \partial_{r}=r \partial / \partial r$ with the spectral parameter $\lambda \in \mathbb{C}$. In what follows, we call the problem (2.4) a (spectral) pencil; to shorten the notation we do not indicate arguments $\varphi$ and $\partial_{\varphi}$ of matrices $\mathfrak{L}, \mathfrak{D}$, and $\mathfrak{N}^{ \pm} ;$in particular, $\mathfrak{L}(\lambda)$ is a quadratic polynomial in $\lambda$ with coefficient at $\lambda^{q}$ being a matrix of differential operators of order $2-q$.

Let us present general information (see [11, 30]) about the spectrum of the pencil (2.4) and some particular consequences (see [3, 29]) of the polynomial property of the operator $\mathbf{L}$. All eigenvalues of the pencil are normal and lie in the closed double sector $\left\{\lambda \in \mathbb{C}:|\operatorname{Im} \lambda| \leq \kappa_{0}|\operatorname{Re} \lambda|\right\}$ for $\kappa_{0} \in \mathbb{R}_{+}$. Each strip $\mathbb{P}_{\delta}=\{\lambda \in \mathbb{C}:|\operatorname{Re} \lambda|<\delta\}$ contains only a finite number of points of the spectrum, and there exists $\delta_{K}>0$ such that the strip $\mathbb{P}_{\delta_{K}}$ contains a single eigenvalue $\lambda_{0}=0$. The spectrum is symmetric with respect to the imaginary axis, and in the case of real matrices $\mathcal{D}, A$, it is also symmetric with respect to the real axis. To each eigenvalue $\lambda_{q}$ there corresponds a canonical sequence of Jordan chains of the pencil (2.4), namely

$$
\left\{\Phi_{(q)}^{j, p}: j=1, \ldots, J_{q} ; p=0, \ldots, \kappa_{q j}-1\right\} ;
$$

here $J_{q}$ and $\kappa_{q j}$ are natural numbers, and $\kappa_{q 1} \geq \cdots \geq \kappa_{q J_{q}}$. Since the vector-valued functions $\Phi_{(q)}^{j, p}$ satisfy the equations

$$
\begin{aligned}
\mathfrak{L}(\lambda) \Phi_{(q)}^{j, p} & =-\frac{\partial \mathfrak{L}}{\partial \lambda}(\lambda) \Phi_{(q)}^{j, p-1}-\frac{1}{2} \frac{\partial^{2} \mathfrak{L}}{\partial \lambda^{2}}(\lambda) \Phi_{(q)}^{j, p-2} \quad \text { on } \Upsilon \\
\mathfrak{N}^{ \pm}(\lambda) \Phi_{(q)}^{j, p} & =-\frac{\partial \mathfrak{N}^{ \pm}}{\partial \lambda}(\lambda) \Phi_{(q)}^{j, p-1} \quad \text { for } \varphi= \pm \alpha
\end{aligned}
$$

the above system is used to construct polynomial-logarithmic solutions of the homogeneous problem (2.1), namely

$$
U_{(q)}^{j, p}(x)=r^{\lambda q} \sum_{s=0}^{p} \frac{1}{s !}(\log r)^{s} \Phi_{(q)}^{j, p-s}(\varphi), \quad j=1, \ldots, J_{q}, \quad p=0, \ldots, \kappa_{q j}-1 .
$$

For $\lambda_{0}=0$ the list (2.5) looks as follows:

$$
U_{(0)}^{j, 0}(x)=e_{j}, \quad U_{(0)}^{j, 1}(x)=e_{j} \log r+\Phi_{(0)}^{j, 1}(\varphi), \quad j=1, \ldots, k .
$$


In other words, $J_{0}=k, \kappa_{0 j}=2$. In formulas (2.6) and in later formulas, $e_{j}=$ $\left(\delta_{j, 1}, \ldots, \delta_{j, k}\right)^{\top}$ and $\delta_{j, k}$ is the Kronecker symbol.

We now introduce the space $V_{\beta}^{l}(\mathbb{K})$ as the completion of the algebraic subspace $C_{c}^{\infty}(\overline{\mathbb{K}} \backslash \mathcal{O})$ with respect to the norm (1.22). For $l \in \mathbb{N}$ we define the trace space $V_{\beta}^{l-1 / 2}(\mathbb{K})$ using the natural rule (1.24). The following results on solvability of the problem (2.1) and the asymptotic formulas for the solutions of this problem are established in 10] (see also [13, Theorems 3.5.1, 3.5.6]).

Proposition 2.1. 1) Let $l \in \mathbb{N}$. The operator of the problem (2.1)

$$
\left\{\mathbf{L}, \mathbf{N}^{ \pm}\right\}: V_{\beta}^{l+1}(\mathbb{K})^{k} \rightarrow V_{\beta}^{l-1}(\mathbb{K})^{k} \times V_{\beta}^{l-1 / 2}(\partial \mathbb{K})^{k}
$$

is an isomorphism if and only if the pencil (2.4) has no eigenvalues on the line $\Lambda_{l-\beta}=$ $\{\lambda \in \mathbb{C}: \operatorname{Re} \lambda=l-\beta\}$. If such eigenvalues exist, then the image of the operator (2.7) is not closed.

2) Let $l \in \mathbb{N}$ and $\beta^{1}, \beta^{2} \in \mathbb{R}$ with $\beta^{1}<\beta^{2}$. We assume that the pencil (2.4) has no eigenvalues on the lines $\Lambda_{l-\beta^{1}}, \Lambda_{l-\beta^{2}}$. Then solutions $u^{i} \in V_{\beta^{i}}^{l+1}(\mathbb{K})^{k}, i=1,2$, of the problem (2.1) with the right-hand side

$$
\{f, g\} \in \bigcap_{i=1,2}\left(V_{\beta^{i}}^{l-1}(\mathbb{K})^{k} \times V_{\beta^{i}}^{l-1 / 2}(\partial \mathbb{K})^{k}\right)
$$

are related by the formula

$$
u^{1}(x)=u^{2}(x)+\sum c_{(q)}^{j, p} U_{(q)}^{j, p}(x),
$$

where the summation is over all eigenvalues of the pencil (2.4) between the lines $\Lambda_{l-\beta^{2}}$ and $\Lambda_{l-\beta^{1}}$, and the $U_{(q)}^{j, p}$ are the corresponding polynomial-logarithmic solutions of (2.5). For the coefficients in formula (2.8) the following estimates hold:

$$
\left|c_{(q)}^{j, p}\right| \leq c \sum_{i=1}^{2}\left(\left\|f ; V_{\beta^{i}}^{l-1}(\mathbb{K})\right\|+\left\|g ; V_{\beta^{i}}^{l-1 / 2}(\partial \mathbb{K})\right\|\right) .
$$

2.2. Spectral problem on an arc in weight classes. For $l=0,1, \ldots$ and $\beta \in \mathbb{R}$ we introduce the spaces $V_{\gamma}^{l}(\Upsilon)$ and $V_{\gamma}^{l+1,1}(\Upsilon)$ of functions on the arc $\Upsilon=(-\alpha, \alpha)$ with the following norms:

$$
\begin{aligned}
\left\|F ; V_{\gamma}^{l}(\Upsilon)\right\| & =\left(\sum_{p=0}^{l}\left\|\theta^{\gamma-l+p} \partial_{\varphi}^{p} F ; L_{2}(\Upsilon)\right\|^{2}\right)^{1 / 2} \\
\left\|U ; V_{\gamma}^{l+1,0}(\Upsilon)\right\| & =\left(\sum_{p=0}^{l+1}\left\|\theta^{\gamma-l-1+p+\delta_{p, 0}} \partial_{\varphi}^{p} U ; L_{2}(\Upsilon)\right\|^{2}\right)^{1 / 2}
\end{aligned}
$$

where $\theta(\varphi)=(2 \alpha)^{-1}\left(\varphi^{2}-\alpha^{2}\right)$ is a weight function equivalent to the distance to the endpoints of the arc. More explicitly, the above spaces contain just those functions $F \in H_{\mathrm{loc}}^{l}(\Upsilon)$ and $U \in H_{\mathrm{loc}}^{l+1}(\Upsilon)$ for which the corresponding norm (2.10) is finite. Using a version of the one-dimensional Hardy inequality (see, e.g., 31) we conclude that under the condition (1.28) for the vector-valued function $U \in V_{\gamma}^{l+1,0}(\Upsilon)^{k}$ the following formulas hold:

$$
\begin{array}{r}
U(\varphi)=\sum_{ \pm} \chi^{ \pm}(\varphi) U( \pm \alpha)+\widetilde{U}(\varphi), \quad \widetilde{U} \in V_{\gamma}^{l+1}(\Upsilon)^{k}, \\
|U(+\alpha)|+|U(-\alpha)|+\left\|\widetilde{U} ; V_{\gamma}^{l+1}(\Upsilon)\right\| \leq c\left\|U ; V_{\gamma}^{l+1,0}(\Upsilon)\right\|,
\end{array}
$$

where $\chi^{ \pm}(\varphi)=\chi(\varphi \mp \alpha)$ are cut-off functions, $\chi \in C_{c}^{\infty}(-\alpha / 2, \alpha / 2)$, and $\chi(t)=1$ for $|t|<\alpha / 4$. Let us emphasize that the space $V_{\gamma}^{l}(\Upsilon)$ can be obtained as the closure of 
the algebraic subspace $C_{c}^{\infty}(\Upsilon)$ in the norm $(2.10)_{1}$ for each weight index $\gamma$; however, according to formulas (2.11), in the case (1.28) the closure of the algebraic subspace $C_{c}^{\infty}(\Upsilon)$ with respect to the norm $\left\|\cdot ; V_{\gamma}^{l+1,0}(\Upsilon)\right\|$ is not the entire space $V_{\gamma}^{l+1,0}(\Upsilon)$ but the subspace $V_{\gamma}^{l+1}(\Upsilon)$.

By definition (2.10), for $U \in V_{\gamma}^{l+1,0}(\Upsilon)^{k}$ we have $\partial_{\varphi} U \in V_{\gamma}^{l}(\Upsilon)^{k}$. Therefore, the vectorvalued functions $\mathfrak{N}^{ \pm}(\lambda) U$ do not have traces at points $\varphi= \pm \alpha$ provided the condition (1.28) is satisfied. (Indeed, if $\varepsilon \in(0, \gamma-l+1 / 2)$, the function $\theta^{-\varepsilon}$ belongs to the space $V_{\gamma}^{l}(\Upsilon)$.) At the same time, under the condition (1.31) we have $V_{\gamma}^{l+1,0}(\Upsilon) \subset H^{1}(\Upsilon)$. Also, using the one-dimensional Hardy inequality "with the logarithm"

$$
\int_{0}^{1} t^{-1}|\log t|^{-2}|z(t)|^{2} d t \leq 4 \int_{0}^{1} t\left|\frac{d z}{d t}(t)\right|^{2} d t, \quad z \in C_{c}^{1}[0,1)
$$

we have the estimates

$$
\begin{aligned}
\left\|\Theta^{-1} V ; L_{2}(\Upsilon)\right\| & \leq c\left\|V ; H^{1}(\Upsilon)\right\|, \\
\left\|\Theta F ; L_{2}(\Upsilon)\right\| & \leq c\left\|F ; V_{\gamma-l+1}^{0}(\Upsilon)\right\| \leq c\left\|F ; V_{\gamma}^{l-1}(\Upsilon)\right\|,
\end{aligned}
$$

and

$$
\left|(F, V)_{\Upsilon}\right| \leq\left\|\Theta F ; L_{2}(\Upsilon)\right\|\left\|\Theta^{-1} V ; L_{2}(\Upsilon)\right\| \leq c\left\|F ; V_{\gamma}^{l-1}(\Upsilon)\right\|\left\|V ; H^{1}(\Upsilon)\right\|,
$$

where $\Theta(\varphi)=\theta(\varphi)(1+|\log \theta(\varphi)|)$. In other words, the Neumann problem

$$
\mathfrak{L}\left(\varphi, \partial_{\varphi}, \lambda\right) U(\varphi)=F(\varphi), \quad \varphi \in \Upsilon, \quad \mathfrak{N}^{ \pm}\left(\partial_{\varphi}, \lambda\right) U( \pm \alpha)=0
$$

with the right-hand side

$$
F \in V_{\gamma}^{l-1}(\Upsilon)^{k}
$$

admits a generalized formulation. Namely, its solution $U \in V_{\gamma}^{l+1,0}(\Upsilon)^{k}$ satisfies a system of ordinary differential equations (2.14) 1 and the integral identity

$$
(\mathbf{A} \mathfrak{D}(\lambda) U, \mathfrak{D}(\bar{\lambda}-1) V)_{\Upsilon}=(F, V)_{\Upsilon}, \quad V \in H^{1}(\Upsilon)^{k} .
$$

It is known that in the case $l \geq 2$ and $F \in H^{l-1}(\Upsilon)^{k} \subset V_{\gamma}^{l-1}(\Upsilon)^{k}$ the generalized solution becomes classical and lies in the space $H^{l+1}(\Upsilon)^{k} \subset V_{\gamma}^{l+1,0}(\Upsilon)^{k}$ (Sobolev spaces belong to weight spaces due to condition (1.31), the Hardy inequality, and the inclusion $H^{3}(\Upsilon) \subset C^{2}(\bar{\Upsilon})$ ). For such a solution the boundary conditions $(2.14)_{2}$ are deduced from identity (2.16). So, the spectra of the problem (2.4) in Sobolev spaces and in weight spaces coincide. Furthermore, we have the following result.

Lemma 2.1. Let a point $\lambda \in \mathbb{C}$ does not belong to the spectrum of the pencil (2.4) and the condition (1.31) is satisfied. Then for each right-hand side (2.15) the problem (2.14) 1 , (2.16) has a unique solution $U \in V_{\gamma}^{l+1,0}(\Upsilon)^{k}$ and

$$
\left\|U ; V_{\gamma}^{l+1,0}(\Upsilon)\right\| \leq c\left\|F ; V_{\gamma}^{l-1}(\Upsilon)\right\| .
$$

Since later we will prove a more general statement, the proof of Lemma 2.1 is omitted. Our immediate goal is to obtain weight estimates for the solutions of problem (2.14) taking into account the dependence of these estimates on the parameter $\lambda$. 
Following [32] and [28, 29] we endow the spaces $V_{\gamma}^{l-1}(\Upsilon)^{k}$ and $V_{\gamma}^{l+1,0}(\Upsilon)^{k}$ with the norms depending on a parameter $\mu \geq 0$,

$$
\begin{array}{r}
\left\|F ; V_{\gamma}^{l-1}(\Upsilon ; \mu)\right\|=\left(\sum_{p=0}^{l-1}\left\|\theta^{\gamma-l+1+p}(1+\theta \mu)^{l-1-p} \partial_{\varphi}^{p} F ; L_{2}(\Upsilon)\right\|^{2}\right)^{1 / 2} \\
\left\|U ; V_{\gamma}^{l+1,0}(\Upsilon ; \mu)\right\|=\left(\sum_{p=1}^{l+1}\left\|\theta^{\gamma-l-1+p}(1+\theta \mu)^{l+1-p} \partial_{\varphi}^{p} U ; L_{2}(\Upsilon)\right\|^{2}\right. \\
\left.+\left\|\theta^{\gamma-l}\left[1+\mu(1+\theta \mu)^{l}\right] U ; L_{2}(\Upsilon)\right\|^{2}\right)^{1 / 2}
\end{array}
$$

Denote the above spaces with the new norm by $V_{\gamma}^{l-1}(\Upsilon ; \mu)^{k}$ and $V_{\gamma}^{l+1,0}(\Upsilon ; \mu)^{k}$ respectively; for $\mu=0$ the difference between norms disappears. If the supports of functions $F$ and $U$ are inside the arc $\Upsilon$, then the norms (2.18) are equivalent, uniformly with respect to $\mu \in[1,+\infty)$, to the norms $\left\|F ; H^{l-1}(\Upsilon ; \mu)\right\|$ and $\left\|U ; H^{l+1}(\Upsilon ; \mu)\right\|$; these are Sobolev norms, but they depend on the above parameter:

$$
\left\|z ; H^{l}(\Upsilon ; \mu)\right\|=\left(\sum_{p=0}^{l} \mu^{2(l-p)}\left\|\partial_{\varphi}^{p} z ; L_{2}(\Upsilon)\right\|^{2}\right)^{1 / 2} .
$$

Local estimates of solutions of elliptic boundary value problems with parameter in these norms are established in [30; these estimates are used, in particular, in the proof of the following result:

Lemma 2.2. Let (1.31) hold and let the number $\lambda \in \mathbb{C}$ satisfy the inequality

$$
|\operatorname{Im} \lambda|>1+\kappa_{1}|\operatorname{Re} \lambda| .
$$

Then there exists $\kappa_{1}>0$ such that the problem (2.16) with the right-hand side (2.9) has a unique solution $U \in V_{\gamma}^{l+1,0}(\Upsilon)^{k}$, for which the following estimate holds:

$$
\left\|U ; V_{\gamma}^{l+1,0}(\Upsilon ;|\lambda|)\right\| \leq c\left\|F ; V_{\gamma}^{l-1}(\Upsilon ;|\lambda|)\right\|,
$$

where the constant depends on neither the solution $U$ nor the parameter $\lambda$ satisfying (2.20).

Proof. According to formula (2.13) the right-hand side of (2.16) is a continuous functional on the space $H^{1}(\Upsilon)^{k} \ni V$. Taking into account what was said in 2.1 about the location of eigenvalues of the pencil (2.4), we conclude that there exists $\kappa_{1}$ such that the point $\lambda$ satisfying (2.20) is not contained in the spectrum of the operator of the problem (2.4), i.e., there exists a unique generalized solution $U \in H^{1}(\Upsilon)^{k}$ of the problem (2.16). Thus, it remains to prove that $U \in V_{\gamma}^{l+1,0}(\Upsilon)^{k}$ and to establish the estimate (2.21). We emphasize that the solution $U$ of an elliptic system with the right-hand side $F \in H_{l o c}^{l-1}(\Upsilon)^{k}$ belongs to the space $H_{\mathrm{loc}}^{l+1}(\Upsilon)^{k}$.

Interpreting the endpoints of the arc $\Upsilon$ as vertices of the one-dimensional cone $\mathbb{R}_{+}$, we verify the necessary facts using the general scheme of [32] (see also 29] regarding the use of the step weight norm $(\underline{2.18})_{2}$ ). To realize this scheme, one has to study model problems of two types: on the line $\mathbb{R}$ and on the half-line $\mathbb{R}_{+}$. Writing the operator $\mathfrak{L}$ in $(2.3)_{1}$ in the form

$$
\mathfrak{L}^{0}\left(\varphi, \partial_{\varphi}, \lambda\right)=\mathfrak{a}^{20}(\varphi) \partial_{\varphi}^{2}+\left(\lambda \mathfrak{a}^{11}(\varphi)+\mathfrak{a}^{10}(\varphi)\right) \partial_{\varphi}+\lambda^{2} \mathfrak{a}^{00}(\varphi),
$$


where the matrix-valued functions $\mathfrak{a}^{p q}$ are smooth on $\bar{\Upsilon}$, we freeze the coefficients at the point $\varphi_{0} \in \Upsilon$ and obtain the first model problem

$$
\begin{gathered}
\mathfrak{L}^{0}\left(\partial_{\psi}, \lambda\right) U(\psi):=\mathfrak{a}^{20}\left(\varphi_{0}\right) \partial_{\psi}^{2} U(\psi)+\lambda \mathfrak{a}^{11}\left(\varphi_{0}\right) \partial_{\psi} U(\psi)+\lambda^{2} \mathfrak{a}^{00}\left(\varphi_{0}\right) U(\psi)=F(\psi), \\
\psi \in \mathbb{R} .
\end{gathered}
$$

Using a result of $[30$, we see that under condition (2.20) this system of differential equations is uniquely solvable for any right-hand side $F \in H^{l-1}(\mathbb{R})^{k}$. In addition, we have the estimate

$$
\left\|U ; H^{l+1}(\mathbb{R} ;|\lambda|)\right\| \leq c\left\|F ; H^{l-1}(\mathbb{R} ;|\lambda|)\right\| .
$$

We recall the equivalence of norms (2.18) and (2.19) discussed before the formulation of the lemma.

Now we freeze the coefficients of the operator (2.22) at the points $\varphi= \pm \alpha$ and make the change of variables $\varphi \mapsto \psi^{ \pm}=|\lambda|(\alpha \mp \varphi)$ and transform the system of equations (2.14) 1 into the system with parameter $\ell=|\lambda|^{-1} \lambda$ lying on the unit circle

$$
\begin{aligned}
& \mathfrak{L}^{ \pm}\left(\psi^{ \pm}, \ell\right) \mathbf{U}\left(\psi^{ \pm}\right) \\
& \quad:=\mathfrak{a}^{20}( \pm \alpha) \partial_{\psi^{ \pm}}^{2} \mathbf{U}\left(\psi^{ \pm}\right) \mp \ell \mathfrak{a}^{11}\left(\psi^{ \pm}\right) \partial_{\psi^{ \pm}} \mathbf{U}\left(\psi^{ \pm}\right)+\ell^{2} \mathfrak{a}^{00}\left(\psi^{ \pm}\right) \mathbf{U}\left(\psi^{ \pm}\right) \\
& \quad=\mathbf{F}\left(\psi^{ \pm}\right), \quad \psi^{ \pm} \in \mathbb{R}_{+} .
\end{aligned}
$$

Accordingly, the integral identity (2.16) is transformed into the identity

$$
\begin{gathered}
q(\mathbf{U}, \mathbf{V} ; \ell):=\left(\mathbf{A} D^{ \pm}\left(\ell, \mp \partial_{\psi^{ \pm}}\right) \mathbf{U}, D^{ \pm}\left(\bar{\ell}, \mp \partial_{\psi^{ \pm}}\right) \mathbf{V}\right)_{\mathbb{R}_{+}}=(\mathbf{F}, \mathbf{V})_{\mathbb{R}_{+}}, \\
V \in C_{c}^{\infty}[0,+\infty)^{k} .
\end{gathered}
$$

We emphasize that $D^{ \pm}\left(\ell, \mp \partial_{\psi^{ \pm}}\right)$and $D^{ \pm}\left(\bar{\ell}, \mp \partial_{\psi^{ \pm}}\right)$are principal terms at the points $\varphi= \pm \alpha$ of the operators $|\lambda|^{-1} \mathfrak{D}\left(\varphi, \partial_{\varphi}, \lambda\right)$ and $|\lambda|^{-1} \mathfrak{D}\left(\varphi, \partial_{\varphi}, \bar{\lambda}-1\right)$, and the vector-valued function $\mathbf{F}$ includes the additional factor $|\lambda|^{2}$ that appeared at the change of variables.

As in [29, 32], instead of the spaces $V_{\gamma}^{l-1}(\Upsilon ;|\lambda|)$ and $V_{\gamma}^{l+1,0}(\Upsilon ;|\lambda|)$ the problem (2.23) is endowed with the spaces $E_{\gamma}^{l-1}\left(\mathbb{R}_{+}\right)$and $E_{\gamma}^{l+1,0}\left(\mathbb{R}_{+}\right)$with the norms

$$
\begin{array}{r}
\left\|\mathbf{F} ; E_{\gamma}^{l-1}\left(\mathbb{R}_{+}\right)\right\|=\left(\sum_{p=0}^{l-1}\left\|\psi^{\gamma-l+1+p}(1+\psi)^{l-1-p} \partial_{\psi}^{p} \mathbf{F} ; L_{2}\left(\mathbb{R}_{+}\right)\right\|^{2}\right)^{1 / 2}, \\
\left\|\mathbf{U} ; E_{\gamma}^{l+1,0}\left(\mathbb{R}_{+}\right)\right\|=\left(\sum_{p=1}^{l+1}\left\|\psi^{\gamma-l-1+p}(1+\psi)^{l+1-p} \partial_{\psi}^{p} \mathbf{U} ; L_{2}\left(\mathbb{R}_{+}\right)\right\|^{2}\right. \\
\left.+\left\|\psi^{\gamma-l}(1+\psi)^{l} \mathbf{U} ; L_{2}\left(\mathbb{R}_{+}\right)\right\|^{2}\right)^{1 / 2} .
\end{array}
$$

In norms (2.25) for $\psi>1$, the weight factors do not depend on the order of the derivative $p$ and are equal to $O\left(|\psi|^{\gamma}\right)$. If $|\lambda| \geq 1$ and the functions $\mathbf{F} \in E_{\gamma}^{l-1}\left(\mathbb{R}_{+}\right)$, $\mathbf{U} \in E_{\gamma}^{l+1,1}\left(\mathbb{R}_{+}\right)$vanish for $\psi>\alpha$, we have

$$
\begin{aligned}
c\left\|\mathbf{F} ; E_{\gamma}^{l-1}\left(\mathbb{R}_{+}\right)\right\| & \leq|\lambda|^{\gamma-l+3 / 2}\left\|\varphi \mapsto \mathbf{F}(|\lambda|(\mp \varphi+\alpha)) ; V_{\gamma}^{l-1}(\Upsilon)\right\| \\
& \leq C\left\|\mathbf{F} ; E_{\gamma}^{l-1}\left(\mathbb{R}_{+}\right)\right\|, \\
c\left\|\mathbf{U} ; E_{\gamma}^{l+1,0}\left(\mathbb{R}_{+}\right)\right\| & \leq|\lambda|^{\gamma-l-1 / 2}\left\|\varphi \mapsto \mathbf{U}(|\lambda|(\mp \varphi+\alpha)) ; V_{\gamma}^{l+1,0}(\Upsilon)\right\| \\
& \leq C\left\|\mathbf{U} ; E_{\gamma}^{l+1,0}\left(\mathbb{R}_{+}\right)\right\|
\end{aligned}
$$

with positive constants $c$ and $C$.

To establish unique solvability of the problem (2.24), we use the trick suggested in 33. Namely, for the test function in the integral identity (2.24) we take the product 
$\mathbf{V}=\mathbf{R} \mathbf{v}$, where, as before, $\mathbf{v} \in C_{c}^{\infty}[0,+\infty)^{k}$, but $\mathbf{R}\left(\psi^{ \pm}\right)=\left(1+\epsilon\left|\psi^{ \pm}\right|^{2}\right)^{\gamma / 2}$ and $\epsilon$ is a positive parameter that will be chosen later. Taking $\mathbf{u}=\mathbf{R}^{-1} \mathbf{U}$ we transform (2.24) to the form

$$
\begin{aligned}
& \left(\mathbf{A} \mathcal{D}^{ \pm}\left(\ell, \mp \partial_{\psi^{ \pm}}\right) \mathbf{u}, \mathcal{D}^{ \pm}\left(\bar{\ell}, \mp \partial_{\psi^{ \pm}}\right) \mathbf{v}\right)_{\mathbb{R}_{+}} \\
& +\left(\mathbf{A} \mathcal{D}^{ \pm}\left(\ell, \mp \partial_{\psi^{ \pm}}\right) \mathbf{u}, \mathbf{R}^{-1}\left[\mathcal{D}^{ \pm}\left(0, \mp \partial_{\psi^{ \pm}}\right), \mathbf{R}\right] \mathbf{v}\right)_{\mathbb{R}_{+}} \\
& -\left(\mathbf{A R}^{-1}\left[\mathcal{D}^{ \pm}\left(0, \mp \partial_{\psi^{ \pm}}\right), \mathbf{R}\right] \mathbf{u}, \mathcal{D}\left(\bar{\ell}, \mp \partial_{\psi^{ \pm}}\right) \mathbf{v}\right)_{\mathbb{R}_{+}} \\
& -\left(\mathbf{A R}^{-1}\left[\mathcal{D}^{ \pm}\left(0, \mp \partial_{\psi^{ \pm}}\right), \mathbf{R}\right] \mathbf{u}, \mathbf{R}^{-1}\left[\mathcal{D}^{ \pm}\left(0, \mp \partial_{\psi^{ \pm}}\right), \mathbf{R}\right] \mathbf{v}\right)_{\mathbb{R}_{+}}=(\mathbf{R} F, \mathbf{v})_{\mathbb{R}_{+}}, \\
& \quad \mathbf{v} \in H^{1}\left(\mathbb{R}_{+}\right)^{k},
\end{aligned}
$$

where $[\mathbf{A}, \mathbf{B}]=\mathbf{A B}-\mathbf{B A}$ is the commutator of the operators $\mathbf{A}$ and $\mathbf{B}$, and

$$
\left[\mathcal{D}^{ \pm}\left(\ell, \mp \partial_{\psi^{ \pm}}\right), \mathbf{R}\right]=\mathcal{D}^{ \pm}\left(0, \mp \partial_{\psi^{ \pm}}\right) \mathbf{R}
$$

is the operator of multiplication by the matrix-valued function. Note that

$$
\left|\mathbf{R}\left(\psi^{ \pm}\right)^{-1}\left[\mathcal{D}^{ \pm}\left(0, \mp \partial_{\psi^{ \pm}}\right), \mathbf{R}\left(\psi^{ \pm}\right)\right]\right| \leq c \epsilon^{1 / 2}\left(1+\epsilon\left|\psi^{ \pm}\right|^{2}\right)^{-1 / 2} .
$$

The operator $\mathfrak{L}^{ \pm}$inherits ellipticity with parameter from the operator $\mathfrak{L}$. Therefore, for a purely imaginary $\ell= \pm i$, the form $q$ satisfies the conditions of the Lax-Milgram lemma, i.e., in particular,

$$
\operatorname{Re} q(\mathbf{u}, \mathbf{u} ; \ell) \geq c\left\|\mathbf{u} ; H^{1}\left(\mathbb{R}_{+}\right)\right\|^{2}, \quad c>0 .
$$

If $\ell$ is a generic complex number, then under condition (2.20) inequality (2.29) remains true for large $\kappa_{1}$. According to (2.28), the sum $s(\mathbf{u}, \mathbf{v} ; \ell)$ of the last three terms on the left-hand side of (2.27) satisfies the following estimate:

$$
\operatorname{Re} s(\mathbf{u}, \mathbf{v} ; \ell) \leq c \epsilon\left\|\mathbf{u} ; H^{1}\left(\mathbb{R}_{+}\right)\right\|\left\|\mathbf{v} ; H^{1}\left(\mathbb{R}_{+}\right)\right\| .
$$

Moreover, the right-hand side of (2.27) is a continuous functional on the Hilbert space $H^{1}\left(\mathbb{R}_{+}\right) \ni \mathbf{v}$, first due to the inclusion $\mathbf{R F} \in L_{2}(1,+\infty)^{k}$ and second, due to an estimate on the interval $(0,1)$ similar to (2.13) that is guaranteed by the Hardy inequality with "logarithm" (2.12). Therefore, under the above-mentioned restrictions the Lax-Milgram lemma yields numbers $\epsilon>0$ and $\kappa_{1}$ such that for each right-hand side $\mathbf{F} \in E_{\gamma}^{l-1}\left(\mathbb{R}_{+}\right)^{k}$ the problem (2.27) has a unique solution $\mathbf{u} \in H^{1}\left(\mathbb{R}_{+}\right)^{k}$ and

$$
\left\|\left(1+\epsilon\left|\psi^{ \pm}\right|^{2}\right)^{\gamma / 2} \mathbf{U} ; H^{1}\left(\mathbb{R}_{+}\right)\right\|=\left\|\mathbf{u} ; H^{1}\left(\mathbb{R}_{+}\right)\right\| \leq c\left\|\mathbf{F} ; E_{\gamma}^{l-1}(\Upsilon)\right\| .
$$

Now we study the behavior of vector-valued functions $\mathbf{U}=\mathbf{R}^{-1} \mathbf{u}$ near the point $\psi^{ \pm}=0$ and at infinity. By the integral identity (2.27) equivalent to the identity (2.24), this function lies in the space $H_{l o c}^{l+1}\left(\mathbb{R}_{+}\right)^{k}$ and satisfies equations (2.23). Local estimate of a solution of the elliptic system yields

$$
\begin{aligned}
& \left\|\mathbf{U} ; H^{l+1}(N, N+1)\right\|^{2} \\
& \quad \leq c\left(\left\|\mathbf{F} ; H^{l-1}(N-1 / 2, N+3 / 2)\right\|^{2}+\left\|\mathbf{U} ; L_{2}(N-1 / 2, N+3 / 2)\right\|^{2}\right),
\end{aligned}
$$

where $N \in \mathbb{N}$. Multiplying inequalities (2.31) by $N^{2 \gamma}$, we can move the expression $\left(1+\psi^{ \pm}\right)^{\gamma}$ inside the norm signs; this is possible due to the relations $1 / 2 \leq N^{-1} \psi^{ \pm} \leq 7 / 2$ on the intervals under consideration. Adding together inequalities (2.31) for all $N \in \mathbb{N}$, we see that the left-hand side becomes finite, due, in particular, to (2.30). Therefore, $\mathbf{U} \in E_{\gamma}^{l+1,0}(1, \infty)^{k}$ and the corresponding norm does not exceed $c\left\|\mathbf{F} ; E_{\gamma}^{l-1}\left(\mathbb{R}_{+}\right)\right\|$. 
The product $\chi_{1} \mathbf{U}$, where $\chi_{1}$ is the cut-off function equal to 1 for $\psi^{ \pm}<1$ and 0 for $\psi^{ \pm}>3 / 2$, satisfies the system

$$
\begin{aligned}
& \mathfrak{a}^{20}( \pm \alpha) \partial_{\psi^{ \pm}}^{2}\left(\chi_{1} \mathbf{U}\right) \\
& =\mathbf{F}^{1}:=\mathbf{F} \pm \ell \mathfrak{a}^{11}\left(\varphi_{0}\right) \partial_{\psi^{ \pm}}\left(\chi_{1} \mathbf{U}\right)-\ell^{2} \mathfrak{a}^{00}\left(\varphi_{0}\right) \chi_{1} \mathbf{U}+\left[\mathfrak{L}^{ \pm}, \chi_{1}\right] \mathbf{U} \\
& \quad \text { for } \psi^{ \pm} \in(0,3 / 2]
\end{aligned}
$$

Therefore,

$$
\chi_{1}\left(\psi^{ \pm}\right) \mathbf{U}\left(\psi^{ \pm}\right)=\int_{\psi^{ \pm}}^{3 / 2}\left(\tau-\psi^{ \pm}\right) \mathfrak{a}^{20}( \pm \alpha)^{-1} \mathbf{F}^{1}(\tau) d \tau .
$$

The right-hand side of (2.32) contains just the function $\mathbf{U}$ and its first derivative, for which $\chi_{1} \mathbf{U}, \partial_{\psi^{ \pm}}\left(\chi_{1} \mathbf{U}\right) \in L_{2}(0,3 / 2)^{k} \subset E_{\gamma-l+1}^{0}(0,3 / 2)^{k}$ according to (1.31). Using a version of the Hardy inequality

$$
\int_{0}^{3 / 2} \psi^{2(\gamma-l)}|\mathbf{Z}(\psi)|^{2} d \psi \leq(2 \gamma-2 l+1)^{-2} \int_{0}^{3 / 2} \psi^{2(\gamma-l+1)}\left|\frac{\partial \mathbf{Z}}{\partial \psi}(\psi)\right|^{2} d \psi, \quad \mathbf{Z} \in C_{c}^{1}\left[0, \frac{3}{2}\right)
$$

applied to the function (2.33) and its derivative, we see that

$$
\begin{aligned}
& \int_{0}^{3 / 2}\left|\psi^{ \pm}\right|^{2(\gamma-l)}\left(\left|\chi_{1} \mathbf{U}\right|^{2}+\left|\partial_{\psi^{ \pm}} \chi_{1} \mathbf{U}\right|^{2}\right) d \psi^{ \pm} \\
& \leq c \int_{0}^{3 / 2}\left|\psi^{ \pm}\right|^{2(\gamma-l)}\left\{\left(\int_{\psi^{ \pm}}^{3 / 2}\left(\tau-\psi^{ \pm}\right) \mathfrak{a}^{20}( \pm \alpha)^{-1} \mathbf{F}^{1}(\tau) d \tau\right)^{2}\right. \\
& \left.\quad+\left(\int_{\psi^{ \pm}}^{3 / 2} \mathfrak{a}^{20}( \pm \alpha)^{-1} \mathbf{F}^{1}(\tau) d \tau\right)^{2}\right\} d \psi^{ \pm} \\
& \leq c \int_{0}^{3 / 2}\left|\psi^{ \pm}\right|^{2(\gamma-l+1)}\left\{\left(\int_{\psi^{ \pm}}^{3 / 2} \mathfrak{a}^{20}( \pm \alpha)^{-1} \mathbf{F}^{1}(\tau) d \tau\right)^{2}+\left|\mathfrak{a}^{20}( \pm \alpha)^{-1} \mathbf{F}^{1}\left(\psi^{ \pm}\right)\right|^{2}\right\} d \psi^{ \pm} \\
& \leq c \int_{0}^{3 / 2}\left|\psi^{ \pm}\right|^{2(\gamma-l+1)}\left|\mathbf{F}^{1}\left(\psi^{ \pm}\right)\right|^{2} d \psi^{ \pm} \leq c\left\|\mathbf{F}^{1} ; E_{\gamma-l+1}^{0}(0,3 / 2)\right\|^{2} \\
& \leq c\left(\left\|\mathbf{F} ; E_{\gamma}^{l-1}\left(\mathbb{R}_{+}\right)\right\|^{2}+\left\|\mathbf{U} ; H^{1}\left(\mathbb{R}_{+}\right)\right\|^{2}\right) .
\end{aligned}
$$

Moreover, the left-hand side majorizes $\left\|\partial_{\psi^{ \pm}} \mathbf{U} ; E_{\gamma-l+1}^{0}(0,1)\right\|^{2}$. Together with (2.32) and the obtained estimate for $\left\|\mathbf{U} ; E_{\gamma}^{l+1}(1,+\infty)\right\|$ this means that $\mathbf{U} \in \mathbf{E}_{\gamma-\mathbf{l}+\mathbf{1}}^{\mathbf{2 , 0}}\left(\mathbb{R}_{+}\right)^{\mathbf{k}}$. If $l>1$, we note that $\mathbf{F}^{1} \in E_{\gamma-l+2}^{1}(0,3 / 2)^{k}$ and, by (2.32), $\mathbf{U} \in E_{\gamma-l+2}^{3,0}\left(\mathbb{R}_{+}\right)^{k}$. After several iterations we get the required estimate $\left\|\mathbf{U} ; E_{\gamma}^{l+1,0}\left(\mathbb{R}_{+}\right)\right\| \leq c\left\|\mathbf{F} ; E_{\gamma}^{l-1}\left(\mathbb{R}_{+}\right)\right\|$for the solution of the model problem (2.27).

Therefore, the general scheme of [29, 32] yields inequality (2.21) for the solution $U \in V_{\gamma}^{l+1,0}(\Upsilon)^{k}$. Finally, in the last part of the proof it was verified that the generalized solution of the problem (2.16) with the right-hand side (2.15) belongs to the space $V_{\gamma}^{l+1,0}(\Upsilon)^{k}$. The lemma is proved.

2.3. Solvability of the model in a sector. After passing to polar coordinates and making the Euler change of variables $r \mapsto t=\log r$, the problem (2.1) with homogeneous boundary conditions takes the form

$$
\begin{gathered}
\mathfrak{L}\left(\varphi, \partial_{\varphi}, \partial_{t}\right) u(\varphi, t)=\exp \{2 t\} f(\varphi, t), \quad \varphi \in \Upsilon, t \in \mathbb{R} ; \\
\mathfrak{N}^{ \pm}\left(\partial_{\varphi}, \partial_{t}\right) u(\varphi, t)=0, \quad t \in \mathbb{R} .
\end{gathered}
$$


We will keep the original notation for vector-valued functions written in coordinates $(\varphi, t)$. Applying the Fourier-Laplace transform

$$
\widehat{u}(\varphi, \lambda)=\mathcal{F}_{t \mapsto \lambda} u(\varphi, t)=\frac{1}{\sqrt{2 \pi}} \int_{\mathbb{R}} \exp \{-t \lambda\} u(\varphi, t) d t
$$

to the problem (2.34) in the strip $\Upsilon \times \mathbb{R}$, we obtain (so far, formally) the following inhomogeneous Neumann problem (2.4) with parameter $\lambda$ on the arc $\Upsilon$ :

$$
\begin{aligned}
\mathfrak{L}\left(\varphi, \partial_{\varphi}, \lambda\right) \widehat{u}(\varphi, \lambda) & =\widehat{f}(\varphi, \lambda-2), & \varphi & \in \Upsilon, \\
\mathfrak{N}^{ \pm}\left(\partial_{\varphi}, \lambda\right) \widehat{u}(\varphi, \lambda) & =0, & t & \in \mathbb{R} .
\end{aligned}
$$

The integral identity

$$
\left(\mathbf{A} \mathcal{D}\left(\nabla_{x}\right) u, \mathcal{D}\left(\nabla_{x}\right) v\right)_{\mathbb{K}}=(f, v)_{\mathbb{K}},
$$

which holds for an arbitrary test function $v \in C_{c}^{\infty}(\mathbb{K})^{k}$, becomes the integral identity (2.16), where we made the changes $U(\varphi) \mapsto \widehat{u}(\varphi, \lambda)$ and $F(\varphi) \mapsto \widehat{f}(\varphi, \lambda-2)$.

We assume that for any point $\lambda$ of the line $\Lambda_{l-\beta}$ containing no eigenvalues of the pencil (2.4), the right-hand side $\widehat{f}(\cdot, \lambda-2)$ belongs to the space $V_{\gamma}^{l-1}(\Upsilon)^{k}$. Due to Lemma 2.2, the solution of the problem (2.36) takes the form $\widehat{u}(\cdot, \lambda)=\mathfrak{R}(\lambda) \widehat{f}(\cdot, \lambda-2)$, where $\mathfrak{R}(\lambda)$ is the resolvent of the operator of the problem. Moreover, the inverse Fourier-Laplace transform

$$
u(\varphi, t)=\mathcal{F}_{\lambda \mapsto t}^{-1} \widehat{u}(\varphi, \lambda)=-\frac{i}{\sqrt{2 \pi}} \int_{\Lambda_{l-\beta}} \exp \{t \lambda\} \widehat{u}(\varphi, \lambda) d \lambda
$$

yields (as before, just formally) a solution of the problem (2.1) with the right-hand sides $f$ and $g=0$. Let us note that for $\lambda \in \Lambda_{l-\beta}$, the real part $\operatorname{Re} \lambda$ is constant and $d \lambda=i d(\operatorname{Im} \lambda)$.

The next theorem shows that under certain conditions, the outlined procedure, which is commonly used in the theory of elliptic boundary value problems in regions with nonsmooth boundaries, yields an isomorphism

$$
\mathbf{L}: \stackrel{\circ}{\mathfrak{V}}_{\beta, \gamma}^{l+1,0}(\mathbb{K})_{\mathfrak{N}}^{k} \rightarrow \stackrel{\circ}{\mathfrak{V}}_{\beta, \gamma}^{l-1}(\mathbb{K})^{k},
$$

corresponding to the problem (2.1) with homogeneous Neumann boundary conditions. The spaces $\mathfrak{V}_{\beta, \gamma}^{l+1,0}(\mathbb{K})$ and $\mathfrak{V}_{\beta, \gamma}^{l-1}(\mathbb{K})$ that are involved in formula (2.38) consist of the functions $u \in H_{\text {loc }}^{l+1}(\mathbb{K})$ and $f \in H_{\text {loc }}^{l-1}(\mathbb{K})$ for which the following weight norms (cf. definitions (1.29), (1.20), and (1.22) ) are finite:

$$
\begin{aligned}
\left\|u ; \mathfrak{V}_{\beta, \gamma}^{l+1,0}(\mathbb{K})\right\| & =\left(\sum_{p=0}^{l+1}\left\|r^{\beta-l-1+p} \theta^{\gamma-l-1+p+\delta_{0, p}} \nabla_{x}^{p} u ; L_{2}(\mathbb{K})\right\|^{2}\right)^{1 / 2} \\
\left\|f ; \mathfrak{\mathfrak { V }}_{\beta, \gamma}^{l-1}(\mathbb{K})\right\| & =\left(\sum_{p=0}^{l-1}\left\|r^{\beta-l+1+p} \theta^{\gamma-l+1+p} \nabla_{x}^{p} f ; L_{2}(\mathbb{K})\right\|^{2}\right)^{1 / 2}
\end{aligned}
$$

The subspace $\mathfrak{V}_{\beta, \gamma}^{l+1,0}(\mathbb{K})_{\mathfrak{N}}^{k}$ contains all vector-valued functions $u \in \mathfrak{V}_{\beta, \gamma}^{l+1,0}(\mathbb{K})^{k}$ satisfying the integral identity (2.37) for $f=\mathbf{L}\left(\nabla_{x}\right) u \in \mathfrak{V}_{\beta, \gamma}^{l-1}(\mathbb{K})^{k}$.

Because of the factor $\theta=(2 \alpha)^{-1}\left(\varphi^{2}-\alpha^{2}\right)$, which vanishes on the sides of the sector $\mathbb{K}$, the spaces $\mathfrak{V}_{\beta, \gamma}^{l+1,0}(\mathbb{K})$ and $\mathfrak{V}_{\beta, \gamma}^{l-1}(\mathbb{K})$ contain (as opposed to the classes $\mathfrak{V}_{\beta, \gamma}^{l}$ and $V_{\beta}^{l}$ introduced in 1.4) elements that do not belong to the algebraic subspaces $H_{\text {loc }}^{l+1}(\overline{\mathbb{K}} \backslash \mathcal{O})$ and $H_{\mathrm{loc}}^{l-1}(\overline{\mathbb{K}} \backslash \mathcal{O})$. 
The verification of the following facts is rather easy. The Euler change of variables $r \mapsto t=\log r$ transforms the spaces $\mathfrak{V}_{\beta, \gamma}^{l+1,0}(\mathbb{K})$ and $\mathfrak{V}_{\beta, \gamma}^{l-1}(\mathbb{K})$ into the spaces $\mathfrak{W}_{\beta-l, \gamma}^{l+1,0}(\mathbb{K})$ and $\mathfrak{W}_{\beta-l, \gamma}^{l-1}(\mathbb{K})$ with the norms

$$
\begin{aligned}
& \left\|u ; \mathfrak{\mathfrak { W }}_{\beta-l, \gamma}^{l+1,0}(\Upsilon \times \mathbb{R})\right\|=\left(\sum_{p=0}^{l+1}\left\|\exp \{(\beta-l) t\} \theta^{\gamma-l-1+p+\delta_{0, p}} \nabla_{(\varphi, t)}^{p} u ; L_{2}(\Upsilon \times \mathbb{R})\right\|^{2}\right)^{1 / 2} \\
& \left\|f ; \mathfrak{\mathfrak { W }}_{\beta-l, \gamma}^{l-1}(\Upsilon \times \mathbb{R})\right\|=\left(\sum_{p=0}^{l-1}\left\|\exp \{(\beta-l+2) t\} \theta^{\gamma-l+1+p} \nabla_{(\varphi, t)}^{p} f ; L_{2}(\Upsilon \times \mathbb{R})\right\|^{2}\right)^{1 / 2}
\end{aligned}
$$

The image of the subspace $\mathfrak{\mathfrak { V }}_{\beta, \gamma}^{l+1,0}(\mathbb{K})_{\mathfrak{N}}^{k}$ is denoted by $\mathfrak{\mathfrak { W }}_{\beta, \gamma}^{b+1,0}(\mathbb{K})_{\mathfrak{N}}^{k}$. By the Parceval identity, the Fourier-Laplace transform (2.35) establishes isomorphisms

$$
\begin{aligned}
& \stackrel{\circ}{\mathfrak{W}}_{\beta-l, \gamma}^{l+1,0}(\Upsilon \times \mathbb{R}) \approx L_{2}\left(\Lambda_{l-\beta} \ni \lambda \mapsto V_{\gamma}^{l+1,0}(\Upsilon ;|\lambda|)\right), \\
& \mathfrak{W}_{\beta-l, \gamma}^{l-1}(\Upsilon \times \mathbb{R}) \approx L_{2}\left(\Lambda_{l-\beta} \ni \lambda \mapsto V_{\gamma}^{l-1}(\Upsilon ;|\lambda|)\right),
\end{aligned}
$$

where $L_{2}\left(\Lambda_{l-\beta} \ni \lambda \mapsto \mathfrak{B}(|\lambda|)\right)$ is the space of functions with values in the Banach space $\mathfrak{B}(|\lambda|)=\mathfrak{B}$, the latter space is endowed with the norm $\|\cdot ; \mathfrak{B}(|\lambda|)\|$ depending on parameter $\lambda$, and

$$
\left\|z ; L_{2}\left(\Lambda_{l-\beta} \ni \lambda \mapsto \mathfrak{B}(|\lambda|)\right)\right\|=\left(\int_{\mathbb{R}}\|z(\cdot, l-\beta+i \eta) ; \mathfrak{B}(1+|\eta|)\|^{2} d \eta\right)^{1 / 2} .
$$

Theorem 2.1. Let $f \in \mathfrak{V}_{\beta, \gamma}^{l-1}(\mathbb{K})^{k}$ with $\gamma$ satisfying the condition (1.31). Let us assume that the line $\Lambda_{l-\beta}$ does not contain eigenvalues of the spectral pencil (2.4). Then the map (2.38) is an isomorphism, i.e., the problem (2.1) with right-hand sides $f$ and $g=0$ has a unique solution $u \in \mathfrak{\mathfrak { V }}_{\beta, \gamma}^{l+1,0}(\mathbb{K})_{\mathfrak{N}}^{k}$ given by the formula

$$
u(\varphi, t)=\mathcal{F}_{\lambda \mapsto t}^{-1} \mathfrak{R}(\lambda) \widehat{f}(\cdot, \lambda-2)
$$

and satisfying the estimate

$$
\left\|u ; \stackrel{\circ}{\mathfrak{V}}_{\beta, \gamma}^{l+1,0}(\mathbb{K})\right\| \leq c_{l, \beta}\left\|f ; \stackrel{\circ}{\mathfrak{V}}_{\beta, \gamma}^{l-1}(\mathbb{K})\right\|,
$$

where the constant $c_{l, \beta}$ depends on the choice of the line $\Lambda_{l-\beta}$ but not on the functions $f$ and $u$.

Proof. Passing to variables $(\varphi, t)$ in formula $(2.40) 1$, using the Parceval identity, and recalling definition $(2.18)$, we obtain

$$
\begin{aligned}
\| f ; & \mathfrak{V}_{\beta, \gamma}^{l-1}(\mathbb{K}) \|^{2} \\
& \sim \sum_{p=0}^{l-1} \sum_{q=0}^{p} \int_{\mathbb{R}} \int_{\Upsilon} \exp \{t(\beta-l+1)\} \theta^{\gamma-l+1+p}\left|\partial_{\varphi}^{q} \partial_{t}^{p-q} f(\varphi, t)\right|^{2} \exp \{2 t\} d \varphi d t \\
& \sim \sum_{q=0}^{l-1} \int_{\Lambda_{l-\beta-2}} \int_{\Upsilon} \theta^{\gamma-l+1+q} \sum_{p=q}^{l-1}(\theta|\lambda|)^{2(p-q)}\left|\partial_{\varphi}^{q} \widehat{f}(\varphi, \lambda)\right|^{2} d \varphi d \lambda \\
& \sim \int_{\Lambda_{l-\beta-2}}\left\|\widehat{f}(\cdot, \lambda) ; V_{\gamma}^{l-1}(\Upsilon ;|\lambda|)\right\|^{2} d \varphi .
\end{aligned}
$$

Here and later, $a \sim b$ means that there are positive constants $c$ and $C$ such that $c a \leq$ $b \leq C a$. Note that $|\lambda| \neq 0$ for $\lambda \in \Lambda_{l-\beta}$ since for $\beta=l$ the line $\Lambda_{0}$ contains the eigenvalue $\lambda=0$ of the pencil (2.4) (see clarifications in 2.1). According to (2.21), the 
solution $\widehat{u}(\cdot, \lambda) \in V_{\gamma}^{l+1,0}(\Upsilon ;|\lambda|)$ of the problem (2.36) with the right-hand side $\widehat{f}(\cdot, \lambda-2) \in$ $V_{\gamma}^{l-1}(\Upsilon ;|\lambda|)$, the last integral in (2.46) gives an upper bound for the following expressions (multiplied by some positive constants):

$$
\begin{aligned}
& \int_{\Lambda_{l-\beta}}\left\|\widehat{u}(\cdot, \lambda) ; V_{\gamma}^{l+1,0}(\Upsilon ;|\lambda|)\right\|^{2} d \varphi \\
& \sim \int_{\Lambda_{l-\beta}} \int_{\Upsilon}\left(\sum_{p=1}^{l+1} \sum_{q=p}^{l+1-p} \theta^{2(\gamma-l+1+p)}(\theta|\lambda|)^{2(q-p)}\left|\partial_{\varphi}^{p} \widehat{u}(\varphi, \lambda)\right|^{2}\right. \\
& \left.\quad+\sum_{q=0}^{l} \theta^{2(\gamma-l)}(\theta|\lambda|)^{2 q}|\lambda|^{2}\left|\partial_{\varphi}^{p} \widehat{u}(\varphi, \lambda)\right|^{2}+\theta^{2(\gamma-l)}|\widehat{u}(\varphi, \lambda)|^{2}\right) d \varphi d \lambda \\
& \sim \int_{\mathbb{R}} \int_{\Upsilon} \exp \{t(\beta-l)\}\left(\sum_{p=1}^{l+1} \sum_{q=p}^{l+1-p} \theta^{2(\gamma-l-1+q)}\left|\partial_{t}^{q-p} \partial_{\varphi}^{p} u(\varphi, t)\right|^{2}\right. \\
& \left.\quad+\sum_{q=0}^{l} \theta^{2(\gamma-l+q)}\left|\partial_{t}^{q+1} u(\varphi, t)\right|^{2}+\theta^{2(\gamma-l)}|u(\varphi, t)|^{2}\right) d \varphi d t \\
& \sim\left\|u ; \mathfrak{V}_{\beta, \gamma}^{l+1,0}(\mathbb{K})\right\|^{2} .
\end{aligned}
$$

The theorem is proved.

Standard arguments using representation (2.44) of the solution $u$ of the problem (2.1) with homogeneous boundary conditions and the Cauchy residue theorem (see [10, and, e.g., 13, 14]) yield the following result.

Theorem 2.2. Let $l \in \mathbb{N}$, the weight $\gamma$ satisfy (1.31), and let $\beta^{1}, \beta^{2} \in \mathbb{R}$ be such that $\beta^{1}<\beta^{2}$ and the lines $\Lambda_{l-\beta^{1}}, \Lambda_{l-\beta^{2}}$ do not contain eigenvalues of the pencil (2.4). Then the solutions $u^{i} \in \mathfrak{V}_{\beta, \gamma}^{l+1,0}(\mathbb{K})_{\mathfrak{N}}^{k}, i=1,2$, of the problem (2.1) with the right-hand sides $f \in \stackrel{\circ}{\mathfrak{V}}_{\beta^{1}, \gamma}^{l-1}(\mathbb{K})^{k} \cap \stackrel{\circ}{\mathfrak{V}}_{\beta^{2}, \gamma}^{l-1}(\mathbb{K})^{k}$ and $g=0$ are related by (2.8), where the summation is over all eigenvalues of the pencil (2.4) between the lines $\Lambda_{l-\beta^{2}}$ and $\Lambda_{l-\beta^{1}}$, and $U_{(q)}^{j, p}$ are the corresponding polynomial-logarithmic solutions of (2.5). For coefficients in formula (2.8) the following estimates hold:

$$
\left|c_{(q)}^{j, p}\right| \leq c\left(\left\|f ; \stackrel{\circ}{\mathfrak{V}}_{\beta^{1}, \gamma}^{l-1}(\mathbb{K})^{k}\right\|+\left\|f ; \stackrel{\circ}{\mathfrak{V}}_{\beta^{2}, \gamma}^{l-1}(\mathbb{K})^{k}\right\|\right) .
$$

2.4. Representations of solutions of the model problem near the sides of the sector. Assume the hypotheses of Theorem 2.1 and for $\lambda \in \Lambda_{l-\beta}$ rewrite formula (2.11) 1 for the solution $\widehat{u}(\cdot, \lambda) \in V_{\gamma}^{l+1,0}(\Upsilon ;|\lambda|)^{k}$ of the problem (2.36) as follows:

$$
\widehat{u}(\varphi, \lambda)=\widehat{u}^{0}(\varphi, \lambda)+\sum_{ \pm} \chi^{ \pm}(\varphi) \chi(|\operatorname{Im} \lambda|(\alpha \mp \varphi)) \widehat{u}( \pm \alpha, \lambda) .
$$

We recall that $\chi^{ \pm}(\varphi)=\chi(\alpha \mp \varphi)$ and $\chi$ is the cut-off function used in (2.11).

Lemma 2.3. We have the following estimate:

$$
\begin{aligned}
& \left\|\widehat{u}^{0}(\cdot, \lambda) ; V_{\gamma}^{l+1}(\Upsilon ;|\lambda|)\right\|+(1+|\lambda|)^{l-\gamma+1 / 2} \sum_{ \pm}|\widehat{u}( \pm \alpha, \lambda)| \\
& \quad \leq c\left\|\widehat{u}(\cdot, \lambda) ; V_{\gamma}^{l+1,0}(\Upsilon ;|\lambda|)\right\|
\end{aligned}
$$

with a constant $c$ independent of the parameter $\lambda \in \Lambda_{l-\beta}$ and the vector-valued function

$$
\widehat{u}(\cdot, \lambda) \in V_{\gamma}^{l+1,0}(\Upsilon ;|\lambda|)^{k} .
$$


Proof. If $|\lambda| \leq \delta$ and $\delta>0$ is small, we can omit the second cutoffs $\chi^{ \pm}(|\operatorname{Im} \lambda|(\alpha \mp \varphi))$ on the right-hand side of (2.48) and derive (2.49) from (2.11) 2 . In the case $\lambda \in \Lambda_{l-\beta}$ and $|\lambda| \geq \delta$ we have

$$
c|\lambda| \leq|\operatorname{Im} \lambda| \leq C|\lambda|
$$

where $c$ and $C$ are positive constants. We rewrite the vector-valued function $\varphi \mapsto$ $\chi^{ \pm}(\alpha \mp \varphi) \widehat{u}(\alpha \mp \varphi, \lambda)$ belonging to the space $V_{\gamma}^{l+1,1}(\Upsilon ;|\lambda|)^{k}$ using the variable $\psi^{ \pm}=$ $|\lambda|(\alpha \mp \varphi)$ and denote the result by $\mathbf{U}^{ \pm}$. Relations (2.50) and $(2.26){ }_{2}$ then show that the estimate (2.49) is guaranteed by the corollary (2.11) of the Hardy inequality for the vector-valued function $\mathbf{U}^{ \pm} \in E_{\gamma}^{l+1,1}\left(\mathbb{R}_{+}\right)^{k}$.

Applying the Fourier-Laplace transform (2.39) inverse to (2.48) we obtain

$$
\begin{aligned}
u(\varphi, t)= & -\frac{i}{\sqrt{2 \pi}} \int_{\Lambda_{l-\beta}} \exp \{t \lambda\} \widehat{u}(\varphi, \lambda) d \lambda \\
& +\frac{1}{\sqrt{2 \pi}} \sum_{ \pm} \chi^{ \pm}(\alpha \mp \varphi) \int_{\mathbb{R}} \exp \{t(l-\beta+i \eta)\} \chi^{ \pm}(|\eta|(\alpha \mp \varphi)) \widehat{u}( \pm \alpha, l-\beta+i \eta) d \eta \\
= & u^{0}(\varphi, t)+\exp \{t(l-\beta)\} \sum_{ \pm} \chi^{ \pm}(\alpha \mp \varphi) \int_{\mathbb{R}} X((\alpha \mp \varphi) \tau) K^{ \pm}(t-\tau)(\alpha \mp \varphi)^{-1} d \tau .
\end{aligned}
$$

Here $u^{0} \in \mathfrak{W}_{\beta-l, \gamma}^{l+1}(\Upsilon \times \mathbb{R})^{k}$ and $X(t), K^{ \pm}(t)$ are the Fourier preimages of the functions $\eta \mapsto \sqrt{2 \pi} \chi(|\eta|)$ and $\eta \mapsto \widehat{u}( \pm \alpha, l-\beta+i \eta)$, respectively, i.e.,

$$
\begin{aligned}
X(t) & =\int_{\mathbb{R}} \exp \{i t \eta\} \chi^{ \pm}(|\eta|) d \eta, \\
K^{ \pm}(t) & =\frac{1}{\sqrt{2 \pi}} \int_{\mathbb{R}} \exp \{i t \eta\} \widehat{u}( \pm \alpha, l-\beta+i \eta) d \eta .
\end{aligned}
$$

Changing the variable, we transform the last integral in (2.51) to the following integral:

$$
\mathfrak{C}^{ \pm}\left(K^{ \pm} ; \varphi, t\right)=\int_{\mathbb{R}} X(z) K^{ \pm}(t-(\alpha \mp \varphi) z) d z .
$$

By definition (2.52) the kernel $X$ of the integral operator (2.53) is a smooth rapidly decreasing function with the unit area under the graph. The latter property guarantees that

$$
\mathfrak{C}^{ \pm}\left(K^{ \pm} ; \pm \alpha, t\right)=K^{ \pm}(t)
$$

Returning to the polar coordinate system, we rewrite representation (2.51) in the following form:

$$
u(x)=u^{0}(x)+r^{l-\beta} \sum_{ \pm} \chi^{ \pm}(\alpha \mp \varphi) \mathfrak{C}^{ \pm}\left(K^{ \pm} ; \varphi, \log r\right) .
$$

Lemma 2.4. The vector-valued functions $u^{0}$ and $K^{ \pm}$satisfy the estimate

$$
\left\|u^{0} ; \mathfrak{V}_{\beta, \gamma}^{l+1}(\Upsilon \times \mathbb{R})\right\|+\sum_{ \pm}\left\|K^{ \pm} ; H^{\kappa}(\mathbb{R})\right\| \leq c\left\|u ; \mathfrak{V}_{\beta, \gamma}^{l+1,0}(\Upsilon \times \mathbb{R})\right\|
$$

where $H^{\kappa}(\mathbb{R})$ is the Sobolev-Slobodetskii space with the smoothness index

$$
\kappa=l-\gamma+\frac{1}{2} \in(0,1) .
$$

Proof. Since the Fourier-Laplace transform establishes isomorphisms (2.42) and the Euler change of variables establishes isomorphisms between the spaces (2.40) and (2.41), Lemma 2.3 implies inequality (2.55). Inclusion (2.56) is guaranteed by (1.31). 
The operator (2.53) determines an extension of functions from the boundary to the interior of the sector $\mathbb{K}$. Next lemma establishes properties of this operator (see [34, 35], and also [13, Propositions 6.4.7, 9.3.10], etc.).

Lemma 2.5. Let $K^{ \pm} \in H^{\kappa}(\mathbb{R})$ and $\kappa \in(0,1)$. Then

$$
\begin{aligned}
\left\|\chi^{ \pm} \mathfrak{C}^{ \pm}\left(K^{ \pm}\right)-\chi^{ \pm} K^{ \pm} ; \mathfrak{\mathfrak { W }}_{0,-\kappa-1 / 2}^{0}(\Upsilon \times \mathbb{R})\right\| \leq c\left\|K^{ \pm} ; H^{\kappa}(\mathbb{R})\right\|, & \\
\left\|\nabla_{(\varphi, t)}^{j} \chi^{ \pm} \mathfrak{C}^{ \pm}\left(K^{ \pm}\right) ; \mathfrak{\mathfrak { W }}_{0, j-\kappa-1 / 2}^{0}(\Upsilon \times \mathbb{R})\right\| \leq c_{j}\left\|K^{ \pm} ; H^{\kappa}(\mathbb{R})\right\|, & j \in \mathbb{N}, \\
\left\|\chi^{ \pm} \mathfrak{C}^{ \pm}\left(K^{ \pm}\right) ; \stackrel{\mathfrak{W}}{0, \varepsilon-1 / 2}_{0}^{0}(\Upsilon \times \mathbb{R})\right\| \leq c_{\varepsilon}\left\|K^{ \pm} ; H^{\kappa}(\mathbb{R})\right\|, & \varepsilon>0 .
\end{aligned}
$$

Due to (1.31) and (2.56), relations (2.57) 3 and (2.57) $)_{2}$ show that the last summand in (2.51) belongs to the space $\mathfrak{W}_{0, \gamma}^{l+1,0}(\Upsilon \times \mathbb{R})^{k}$, as should have been expected.

2.5. Model problem in a half-strip. In the set $G_{R}$ we pass to coordinates $y^{ \pm}$(see relations (1.1) and (1.2)). Under the assumption (1.15) we transform the operators (1.5) and (1.11) into the operators

$$
L\left(\nabla_{y^{ \pm}}\right)=\mathcal{D}^{ \pm}\left(\nabla_{y^{ \pm}}\right)^{*} \mathbf{A} \mathcal{D}^{ \pm}\left(\nabla_{y^{ \pm}}\right), \quad N^{ \pm}\left(y^{ \pm}, \nabla_{y^{ \pm}}\right)={\overline{\mathcal{D}\left(\nu^{ \pm}(y)\right)}}^{\top} \mathbf{A} \mathcal{D}^{ \pm}\left(\nabla_{y \pm}\right) ;
$$

here $\mathcal{D}^{ \pm}\left(\nabla_{y^{ \pm}}\right)$is the image of the matrix $\mathcal{D}\left(\nabla_{x}\right)$ of the differential operators under the above change of variables. As we have already mentioned in 1.5, near each periodic curve $\Gamma_{R}^{ \pm}$on the boundary of the region $G$ we have a boundary layer phenomenon that is described by the following problem in a half-strip:

$$
\Pi^{ \pm}=\left\{y^{ \pm}: y_{1}^{ \pm} \in(0,1), y_{2}^{ \pm}>H^{ \pm}\left(y_{1}^{ \pm}\right)\right\}
$$

with the curved base

$$
\begin{aligned}
L^{ \pm}\left(\nabla_{y^{ \pm}}\right) v^{ \pm}\left(y^{ \pm}\right) & =f^{ \pm}\left(y^{ \pm}\right), & & y^{ \pm} \in \Pi^{ \pm}, \\
N^{ \pm}\left(y^{ \pm}, \nabla_{y^{ \pm}}\right) v^{ \pm}\left(y^{ \pm}\right) & =g^{ \pm}\left(y^{ \pm}\right), & & y^{ \pm} \in \pi^{ \pm} .
\end{aligned}
$$

We supplement the Neumann boundary conditions on $\pi^{ \pm}=\left\{y^{ \pm}: y_{1}^{ \pm} \in(0,1), y_{2}^{ \pm}=\right.$ $\left.H^{ \pm}\left(y_{1}\right)\right\}$ with the following periodicity conditions on the sides of the half-strip:

$$
v^{ \pm}\left(0, y_{2}^{ \pm}\right)=v^{ \pm}\left(1, y_{2}^{ \pm}\right), \quad \nabla_{y^{ \pm}} v^{ \pm}\left(0, y_{2}^{ \pm}\right)=\nabla_{y^{ \pm}} v^{ \pm}\left(1, y_{2}^{ \pm}\right), \quad \pm y_{2}^{ \pm}>H^{ \pm}(0) .
$$

By $W_{\sigma, p e r}^{l}\left(\Pi^{ \pm}\right)$we mean the complement with respect to the weight norm

$$
\left\|Z^{ \pm} ; W_{\sigma}^{l}\left(\Pi^{ \pm}\right)\right\|=\left(\sum_{p=0}^{l}\left\|\exp \left\{\sigma y_{2}^{ \pm}\right\} \nabla_{y^{ \pm}} Z ; L_{2}\left(\Pi^{ \pm}\right)\right\|^{2}\right)^{1 / 2}
$$

of the algebraic subspace $C_{c, \text { per }}^{\infty}\left(\bar{\Pi}^{ \pm}\right)$of smooth 1-periodic functions in $y_{1}^{ \pm}$with compact support. Since $\pi^{ \pm}$is a bounded set, the trace space for $W_{\sigma, \text { per }}^{l}\left(\Pi^{ \pm}\right)$is the SobolevSlobodetskii space $H_{\mathrm{per}}^{l-1 / 2}\left(\Pi^{ \pm}\right)$of periodic functions. Let us emphasize that in the case $\sigma>0$ the imbedding $z^{ \pm} \in W_{\sigma \text {, per }}^{l}\left(\Pi^{ \pm}\right)$implies that $z^{ \pm}\left(y^{ \pm}\right)$decreases exponentially as $y_{2}^{ \pm} \rightarrow+\infty$.

In [3, 36 it is established that the polynomial property of the operator $L^{ \pm}$guarantees the following result on vanishing at infinity of solutions of the problem (2.59), (2.60).

Proposition 2.2. Let $l \in \mathbb{N}, \sigma \in \mathbb{R}_{+}$, and let the right-hand side

$$
\left\{f^{ \pm}, g^{ \pm}\right\} \in W_{\sigma, \text { per }}^{l-1}\left(\Pi^{ \pm}\right)^{k} H_{\text {per }}^{l-1 / 2}\left(\pi^{ \pm}\right)^{k}
$$

of the problem (2.59), (2.60) satisfy the condition

$$
\int_{\Pi^{ \pm}} f^{ \pm}\left(y^{ \pm}\right) d y^{ \pm}+\int_{\pi^{ \pm}} g^{ \pm}\left(y^{ \pm}\right) d s_{y^{ \pm}}=0 \in \mathbb{C}^{k}
$$


Then there exists $\sigma_{\Pi}>0$ such that for $\sigma \in\left(0, \sigma_{\Pi}\right)$ the problem (2.59), (2.60) has a unique solution $v^{ \pm} \in W_{\sigma, \mathrm{per}}^{l+1}\left(\Pi^{ \pm}\right)$and

$$
\left\|v^{ \pm} ; W_{\sigma}^{l+1}\left(\Pi^{ \pm}\right)\right\| \leq c\left(\left\|f^{ \pm} ; W_{\sigma}^{l-1}\left(\Pi^{ \pm}\right)\right\|+\left\|g^{ \pm} ; H_{\mathrm{per}}^{l-1 / 2}\left(\pi^{ \pm}\right)\right\|\right) .
$$

If $v^{ \pm} \in W_{\tau, \text { per }}^{l+1}\left(\Pi^{ \pm}\right)$is a solution of the homogeneous problem (2.59), (2.60) and $\tau \in\left(-\sigma_{\Pi}, 0\right)$, then $v^{ \pm}$is a constant vector. If the solvability conditions (2.61) are not satisfied, then each solution of the problem (2.59), (2.60) grows at least linearly at infinity.

Finally, let us note that the necessity of solvability conditions (2.61) can be verified, for example, by substituting a solution $v^{ \pm}$and constant vectors $e_{1}, \ldots, e_{k}$ (the standard basis in the Euclidean space $\mathbb{R}^{k}$ ) into the Green formula (similar to (1.14)) adapted to the problem (2.59), (2.60).

\section{Formal Asymptotics of SOlutions}

3.1. Sector with periodic boundary. As we have mentioned in 1.3, the structure of the asymptotics at infinity of a solution of the problem (1.10) is more complicated than that of the pure problem in a sector: boundary layers do appear near the periodic boundary. In this section we show how one can use asymptotic blocks

$$
\mathcal{U}(x)=r^{\lambda} \Phi(\varphi, \log r)+r^{\lambda-1} \sum_{ \pm} \chi^{ \pm}(\varphi) \mathcal{V}^{ \pm}\left(y^{ \pm}, \log r\right), \quad x \in G_{R},
$$

containing different asymptotic terms to compensate the following main terms (also multiscale) in the asymptotic expansion of the right-hand side at infinity:

$$
\begin{aligned}
f(x) & =r^{\lambda-2} \Psi(\varphi, \log r)+r^{\lambda-1} \sum_{ \pm} \chi^{ \pm}(\varphi) \mathcal{F}^{ \pm}\left(y^{ \pm}, \log r\right)+\cdots, \\
g^{ \pm}(x) & =r^{\lambda-1} \mathcal{G}^{ \pm}\left(y^{ \pm}, \log r\right)+\cdots .
\end{aligned}
$$

Here and later, dots denote the summands that are not essential in the formal procedure. An important feature is that new mismatches caused by the vector-valued function (3.1) in the system of equations on $G$ and the boundary conditions on $\Gamma_{R}^{ \pm}$can still be expanded in asymptotic series with terms similar to (3.2) but with smaller exponents $\lambda-1, \lambda-2, \ldots$ instead of $\lambda$.

Let us mention properties of coefficients in representations (3.1) and (3.2). They depend polynomially on $\log r$, smoothly on the angle variable $\varphi \in[-\pi, \pi]$, and the dependence on the variables $y^{ \pm} \in \overline{\Pi^{ \pm}}$(or $y^{ \pm} \in \overline{\pi^{ \pm}}$in the case of $\left.(\underline{3.2})_{2}\right)$ is smooth and periodic in $y_{1}^{ \pm}$; moreover, the terms $\mathcal{V}^{ \pm}\left(y^{ \pm}, l\right)$ and $\mathcal{F}^{ \pm}\left(y^{ \pm}, l\right)$ decrease exponentially as $y_{2}^{ \pm} \rightarrow+\infty$. The number $\lambda \in \mathbb{C}$ is arbitrary, and by $\chi^{ \pm}$we mean the cutoffs discussed in Section 2 after formula (2.11). Vector-valued functions $\varphi \mapsto \Phi(\varphi, l), \Psi(\varphi, l)$ are smoothly extended to the neighborhood of the segment $\bar{\Upsilon}$, so that the first summand on the righthand side of (3.1) and of (3.2) 1 are defined everywhere on $G_{R}$.

We substitute Ansatzen (3.1) and (3.2) in the system of equations of problem (1.10) restricted to the region $G_{R}$ and collect factors with equal powers of $r$ written in coordinates $(r, \varphi)$ or $y^{ \pm}$. First of all, we receive the following system of differential equations in the sector:

$$
\mathbf{L}\left(\nabla_{x}\right) r^{\lambda} \Phi(\varphi, \log r)=r^{\lambda-2} \Psi(\varphi, \log r), \quad x \in \mathbb{K}_{R} .
$$

Furthermore, we obtain systems of differential equations in half-strips $\Pi^{ \pm}$,

$$
L^{ \pm}\left(\partial_{y^{ \pm}}\right) \mathcal{V}^{ \pm}\left(y^{ \pm}, l\right)=\mathcal{F}^{ \pm}\left(y^{ \pm}, l\right), \quad y_{1}^{ \pm} \in(0,1), y_{2}^{ \pm}>H^{ \pm}\left(y_{1}^{ \pm}\right)
$$


where, in contrast with (3.3), the argument $l=\log r$ is viewed as a parameter and the differential $\partial_{y^{ \pm}}$does not involve this parameter. The explanation is that the function $x \mapsto r^{\lambda-1} \mathcal{V}^{ \pm}\left(y^{ \pm}, \log r\right)$ depends on $r$ and $y_{i}^{ \pm}$in two ways: differentiation with respect to $r$ decreases the order of growth $\operatorname{Re} \lambda-1$ by one, whereas differentiation with respect to $y_{i}^{ \pm}$does not change this order 2 This is why on the left-hand side of (3.4) and in later formulas we use the "partial" gradient $\partial_{y^{ \pm}}$instead of the "full" gradient.

Consider boundary conditions on $\Gamma_{R}^{ \pm}$. Note that for $H_{0}>0$, on the set $\{x: r>R$, $\left.\left|y_{2}^{ \pm}\right| \leq H_{0}\right\}$ we have the formula

$$
r(\alpha \mp \varphi)=y_{2}^{ \pm}+O(1) .
$$

Therefore,

$$
\begin{aligned}
r^{\lambda} \Phi(\varphi, \log r)= & r^{\lambda} \Phi( \pm \alpha, \log r) \mp r^{\lambda-1} y_{2}^{ \pm} \partial_{\varphi} \Phi( \pm \alpha, \log r) \\
& +O\left(r^{\operatorname{Re} \lambda-2}|\log r|^{\operatorname{deg} \Phi}\right) \quad \text { as } \quad r \rightarrow \infty
\end{aligned}
$$

Consequently, using the second formula in (2.58), we can deduce the following relations on the lines $\Gamma_{R}^{ \pm}$:

$$
\begin{aligned}
N^{ \pm}\left(y^{ \pm}, \nabla_{y^{ \pm}}\right) r^{\lambda} \Phi(\varphi, \log r)= & {\overline{D^{ \pm}\left(\nu^{ \pm}\left(y^{ \pm}\right)\right)}}^{\top} r^{\lambda-1} \phi^{ \pm}(\log r)+\cdots, \\
r^{\lambda-1} \phi^{ \pm}(\log r)=A\left\{D^{ \pm}\left(\partial_{r}, 0\right) r^{\lambda} \Phi( \pm \alpha, \log r)\right. & \left.\quad+r^{\lambda-1} D^{ \pm}(0,1) \partial_{\varphi} \Phi( \pm \alpha, \log r)\right\} \\
= & \left.A D\left(\nabla_{x}\right) r^{\lambda} \Phi(\varphi, \log r)\right|_{\varphi= \pm \alpha} .
\end{aligned}
$$

Therefore, the boundary condition for the vector-valued function $\mathcal{V}^{ \pm}$looks as follows:

$$
N^{ \pm}\left(y^{ \pm}, \partial_{y^{ \pm}}\right) \mathcal{V}^{ \pm}\left(y^{ \pm}, l\right)=\mathcal{G}^{ \pm}\left(y^{ \pm}, l\right)-{\overline{D^{ \pm}\left(\nu^{ \pm}\left(y^{ \pm}\right)\right.}}^{\top} \phi^{ \pm}(l), \quad y^{ \pm} \in \pi^{ \pm} .
$$

Similarly to 2.2 , the problem for the boundary layer is completed by periodicity conditions of the type (2.60).

According to Proposition 2.2, a solution of the problem (3.4), (3.8), (2.60) exponentially decreasing at infinity exists only in the case where the orthogonality conditions (2.61) are satisfied; note that in our case these conditions take the form

$$
\Psi^{ \pm}(l):=\int_{\Pi^{ \pm}} \mathcal{F}^{ \pm}\left(y^{ \pm}, l\right) d y^{ \pm}+\int_{\pi^{ \pm}} \mathcal{G}^{ \pm}\left(y^{ \pm}, l\right) d s_{y}^{ \pm}=\int_{\pi^{ \pm}}{\overline{\mathcal{D}^{ \pm}\left(\nu^{ \pm}\left(y^{ \pm}\right)\right)}}^{\top} d s_{y^{ \pm}} \phi^{ \pm}(l) .
$$

By the Stokes formula,

$$
\begin{aligned}
\int_{\pi^{ \pm}}{\overline{\mathcal{D}^{ \pm}\left(\nu^{ \pm}\left(y^{ \pm}\right)\right)}}^{\top} d s_{y^{ \pm}} & =-\int_{\pi^{ \pm}}{\overline{\mathcal{D}^{ \pm}(0,1)}}^{\top} d s_{y^{ \pm}} \\
& =-\int_{\pi\left( \pm H_{0}\right)} \overline{\mathcal{D}(\sin \alpha, \mp \cos \alpha)} d y_{2}^{ \pm}=\mathcal{D}\left(\nu^{ \pm}\right)
\end{aligned}
$$

where $\pi\left(H_{0}\right)=\left\{y^{ \pm}: y_{1}^{ \pm} \in(0,1), y_{2}^{ \pm}=H_{0}\right\}$ is a cross-section of the half-strip $\Pi^{ \pm}$that is located far from the base, and $\nu^{ \pm}=(-\sin \alpha, \pm \cos \alpha)^{\top}$ are outward unit normals to the sides $(\partial \mathbb{K})^{ \pm}$of the sector.

Hence, formulas (3.9) become the Neumann boundary conditions

$$
\mathbf{N}\left(\nabla_{x}\right) r^{\lambda} \Phi(\varphi, \log r)=r^{\lambda-1} \Psi^{ \pm}(\log r), \quad \varphi= \pm \alpha .
$$

\footnotetext{
${ }^{2}$ In the language of averaging theory, the variables $r$ and $\varphi$ are declared to be slow, and the variables $y^{ \pm}$, to be fast.
} 
It is known (see [10, as well as [13, Theorem 3.5.13]) that the model problem (3.3), (3.10) with the special right-hand side always has a solution $r^{\lambda} \Phi(\varphi, \log r)$; moreover,

$$
\operatorname{deg} \Phi=\max \left\{\operatorname{deg} \Psi, \operatorname{deg} \Psi^{ \pm}\right\}+\kappa(\lambda),
$$

where $\operatorname{deg} \Phi$ (resp., $\operatorname{deg} \Psi)$ is the degree of the polynomial $\Phi(\varphi, \log r)$ (resp., the polynomial $\Psi(\varphi, \log r))$ in variable $\log r$, and $\kappa(\lambda)=\max \left\{\kappa_{q 1}, \ldots, \kappa_{q J_{q}}\right\}$ if $\lambda=\lambda_{q}, \kappa(\lambda)=0$ if $\lambda$ is not an eigenvalue of the pencil (2.4).

After finding the solution of the problem in the sector $\mathbb{K}$, we look for solutions $\mathcal{V}^{ \pm}$ in half-strips $\Pi^{ \pm}$; these solutions vanish exponentially at infinity due to orthogonality conditions (3.9).

Let us discuss additional mismatches that are introduced by the asymptotic solution (3.1) in the problem (1.10). The first summand $r^{\lambda} \Phi$ in (3.1) completely compensates the term $r^{\lambda-2} \Psi$ in the representation $(3.2)_{1}$; however, it adds the sum of the following terms to the boundary conditions on lines $\Gamma_{R}^{ \pm}$:

$$
r^{\lambda-1-j} \mathcal{G}^{j \pm}\left(y^{ \pm}, \log r\right), \quad j=1,2, \ldots .
$$

These terms appear when we expand the relations

$$
y_{1}^{ \pm}=r \cos (\alpha \mp \varphi), \quad y_{2}^{ \pm}=r \sin (\alpha \mp \varphi)
$$

between "fast" and "slow" variables in series in inverse powers of the radius $r$ (cf. formula (3.5)), and they are generated by lower terms in representations (3.6) and (3.7) $)_{1}$. It is clear that an infinite series representation for the trace of the vector-valued function $N^{ \pm} r^{\lambda} \Phi \Gamma_{R}^{ \pm}$can be obtained only in the case where $\Phi(\cdot, \log r) \in C^{\infty}[-\pi, \pi]^{k}$ and $H^{ \pm} \in$ $C^{\infty}\left(\mathbb{R}_{+}\right)$. Let us emphasize that the expressions in the middle part of $(3.7)_{2}$ (which, at the end, make up the Neumann boundary value operator (3.10)) are of the form (3.11) with $j=-1$ and $j=0$. The exponents of the radius $r$ in the vector-valued function (3.11) are smaller than on the right-hand side of $(3.2)_{2}$.

The appearance of mismatches caused by the second summand in (3.1) is a consequence of the fact that in forming the limiting problems (3.4), (3.8) we have assumed that the operators $L^{ \pm}$and $N^{ \pm}$do not act on the arguments $r$ and $\log r$. Indeed,

$$
\begin{aligned}
\nabla_{y^{ \pm}} & \left(r^{\lambda-1} \mathcal{V}^{ \pm}\left(y^{ \pm}, \log r\right)\right) \\
= & \left.\partial_{y^{ \pm}}\left(\rho^{\lambda-1} \mathcal{V}^{ \pm}\left(y^{ \pm}, \log \rho\right)\right)\right|_{\rho=r} \\
& \quad+\left.\left(\left[1-r^{-2}\left(y_{2}^{ \pm}\right)^{2}\right]^{1 / 2}, r^{-1} y_{2}^{ \pm}\right)^{\top} \partial_{r}\left(r^{\lambda-1} \mathcal{V}^{ \pm}\left(\eta^{ \pm}, \log r\right)\right)\right|_{\eta^{ \pm}=y^{ \pm}} .
\end{aligned}
$$

The first term on the right is of order $r^{\operatorname{Re} \lambda-1}$, and the second is of order $r^{\operatorname{Re} \lambda-2}$; moreover, the factor

$$
\left(\left[1-r^{-2}\left(y_{2}^{ \pm}\right)^{2}\right]^{1 / 2}, r^{-1} y_{2}^{ \pm}\right)^{\top}
$$

is computed according to the last formula (3.12) (we cannot use the variable $y_{1}^{ \pm}$since all summands on the right-hand side of (3.13) depend on the variable $y_{1}^{ \pm}$periodically). Taking into account formula (3.13) in computing the action of the operators (2.58) on the product $\chi^{ \pm}(\varphi) r^{\lambda-1} \mathcal{V}^{ \pm}\left(y^{ \pm}, \log r\right)$, we can easily compute the discrepancies under consideration. Note that the terms occurring when taking the commutator of the cutoff $\chi^{ \pm}$with the operator $L^{ \pm}\left(\nabla_{x}\right)$ are supported in the sector $\{x: r>R, \pm \varphi \in(\alpha / 2,3 \alpha / 4)\}$, and hence decrease exponentially with rate $o\left(\exp \left\{-(2 r)^{-1} \sigma_{\Pi}\right\}\right)$ at infinity (because of the same property of the boundary layer, Proposition 2.2). In other words, the presence of cut-off functions $\chi^{ \pm}$in (3.1) does not lead to any modification in the procedure of constructing the asymptotics. 
3.2. Once again about the structure of asymptotics. In Section 4 the problem (1.10) in the sector with periodic boundary will be reduced to a model problem (2.1) in the sector $\mathbb{K}$, after which Proposition 2.1, 1) will be applied. Power solutions of the model problem (2.1) singled out in formula (2.8) should have been taken into account in finding the block (3.1) in the the previous subsection: if $\lambda=\lambda_{q}$ is an eigenvalue for the problem (2.4), then a solution $r^{\lambda} \Phi$ of the problem (3.3), (3.8) is determined uniquely up to a linear combination of power solutions (2.5) and can be nonzero even in the case $\Psi=0$ and $\mathcal{F}^{ \pm}=0, \mathcal{G}^{ \pm}=0$. The coefficients of this linear combination cannot be determined in the process of constructing formal asymptotics: they depend on the data of the problem in general, and it is Proposition 2.1, 1), together with the estimate (2.9), that determines the correct values of these coefficients (see also 5.3).

Let the functions the right-hand sides of the problem (1.10) vanish sufficiently fast at infinity (for example, are compactly supported). Suppose we somehow know that the asymptotic expansion of a solution $u$ contains the term $U_{(q)}^{j, p}(x)=r^{\lambda_{q}} \Phi_{(q)}^{(j, p)}(\varphi, \log r)$ (cf. formula (2.5)). To simplify the analysis, let us assume for now that the set (1.1) is contained in the set $\mathbb{K}_{R}=\{x \in \mathbb{K}: r>R\}$, i.e., $H^{ \pm} \geq 0$. We introduce the cut-off function $\chi_{\infty} \in C^{\infty}\left(\mathbb{R}_{+}\right)$satisfying the relations $\chi_{\infty}(r)=1$ for $r>3 R$ and $\chi_{\infty}(r)=0$ for $r<2 R$ and consider the difference

$$
u^{\prime}(x)=u(x)-\chi_{\infty}(r) U_{(q)}^{j, p}(x) .
$$

One can easily see that the second term lies in the space $\mathfrak{V}_{\beta, \gamma}^{l+1,0}(\Omega)^{k}$ for each smoothness index and for each weight indices $\gamma$ and $\beta$ satisfying the conditions (1.31) and

$$
\beta<l-\operatorname{Re} \lambda_{q}
$$

According to (1.15) and (2.2), the support of the vector-valued function $L \chi_{\infty} U_{(q)}^{j, p}$, where $L$ is the operator (1.5), is compact, i.e., $L \chi_{\infty} U_{(q)}^{j, p} \in \mathfrak{V}_{\beta, \gamma}^{l-1}(\Omega)^{k}$ for all $l \in \mathbb{N}$ and $\beta, \gamma \in \mathbb{R}$. At the same time, due to the oscillation of the boundary, the term $N \chi_{\infty} U_{(q)}^{j, p}$, where $N$ is the operator (1.11), turns out to lie in the space $\mathfrak{V}_{\beta, \gamma}^{l-1 / 2}(\partial \Omega)^{k}$ for each $l \in \mathbb{N}$ and $\gamma \in(l-1 / 2, l)$; however, the index $\beta$, which is responsible for the behavior of functions at infinity, must satisfy the relation

$$
\beta<\gamma-\operatorname{Re} \lambda_{q}+\frac{1}{2}
$$

this results from the relation (1.31), which is essentially similar to the relation (3.15). In this situation we cannot hope to improve the estimate of the remainder $u^{\prime}$. Therefore, for the main term of asymptotics it is reasonable to take the block

$$
\mathcal{U}_{(q)}^{j, p}(x)=U_{(q)}^{j, p}(x)+r^{\lambda_{q}-1} \sum_{ \pm} \chi^{ \pm}(\varphi) \mathcal{V}_{(q)}^{j, p \pm}\left(y^{ \pm}, \log r\right)
$$

similar to (3.1); instead of the difference (3.14) it is natural to take the difference

$$
\widetilde{u}(x)=u(x)-\chi_{\infty}(r) \mathcal{U}_{(q)}^{j, p}(x),
$$

where $\mathcal{V}_{(q)}^{j, p \pm}$ are (exponentially decaying as $y_{2}^{ \pm} \rightarrow+\infty$ ) solutions of the problems (3.4), (3.8), (2.60), where $\mathcal{F}^{ \pm}=0, \mathcal{G}^{ \pm}=0$, and the expressions $\phi^{ \pm}$are found according to formula (3.7) 2 using the polynomial logarithmic solution $U_{(q)}^{j, p}$. We emphasize that the existence of exponentially decreasing solutions $\mathcal{V}_{(q)}^{j, p \pm}$ is guaranteed by formulas (3.7)(3.10) and the homogeneous boundary conditions $\mathbf{N}^{ \pm} U_{(q)}^{j, p}=0$ on $(\partial \mathbb{K})^{ \pm}$. Now the inclusions

$$
L \chi_{\infty} \mathcal{U}_{(q)}^{j, p} \in \mathfrak{V}_{\beta, \gamma}^{l-1}(\Omega)^{k}, \quad N \chi_{\infty} \mathcal{U}_{(q)}^{j, p} \in \mathfrak{V}_{\beta, \gamma}^{l-1 / 2}(\partial \Omega)^{k}
$$


hold for all exponents $l \in \mathbb{N}$, and all $\gamma$ and $\beta$ satisfying the conditions (1.31) and

$$
\beta<\gamma-\operatorname{Re} \lambda_{q}+\frac{3}{2} \text {. }
$$

For further exposition it is important to note that the right-hand side of (3.18) is larger by at least 1 than the right-hand side of (3.15) and of (3.16). In other words, the blocks (3.17) constructed using the solutions (2.5) satisfy the homogeneous problem (1.10) with sufficient precision.

Similar constructions are necessary in the case where the inclusion $G_{R} \subset \mathbb{K}_{R}$ does not hold; in this case the angular part $\Phi_{(q)}^{(j, p)}$ of the vector-valued function $U_{(q)}^{j, p}$ on the righthand side of (3.17) should be smoothly extended to the neighborhood of the segment $\bar{\Upsilon}=[-\alpha, \alpha]$.

3.3. Sector with parabolic inclusions. We choose the asymptotic Ansatz for the solution of the problem (1.13) in the compound region $\Omega^{0} \cup \Gamma \cup \Omega^{1}$ in the following form:

$$
\begin{aligned}
\mathcal{U}^{0}(x) & =r^{\lambda} \Phi(\varphi, \log r), \quad x \in \Omega_{R}^{0}, \\
\mathcal{U}^{ \pm}(x) & =\left(y_{1}^{ \pm}\right)^{\lambda} \Phi\left( \pm \alpha, \log y_{1}^{ \pm}\right)+\left(y_{1}^{ \pm}\right)^{\lambda-1+s} \mathcal{V}^{ \pm}\left(\zeta^{ \pm}, \log y_{1}^{ \pm}\right), \quad x \in \Omega_{R}^{ \pm} .
\end{aligned}
$$

Here $\zeta^{ \pm}=\left(y_{1}^{ \pm}\right)^{-s} y_{2}^{ \pm}$is a "fast" variable: the parts of the boundary of the parabolic set $\Omega_{R}^{ \pm}$defined by (1.3) are given by equations $\zeta^{ \pm}=0$ and $\zeta^{ \pm}=-a_{ \pm}$. To ensure that the described procedure of constructing the terms of Ansatz (3.19) allows us to determine the entire asymptotic series, it is convenient to allow for inhomogeneity $\mathcal{G}^{0}$ in the second matching condition (1.13) 2 . Ansatzen for the right-hand sides in the problem (1.13) look as follows:

$$
\begin{aligned}
f^{0}(x) & =r^{\lambda-2} \Psi(\varphi, \log r)+\cdots, \quad x \in \Omega_{R}^{0}=\mathbb{K}_{R}, \\
f^{ \pm}(x) & =\left(y_{1}^{ \pm}\right)^{\lambda-s-1} \mathcal{F}^{ \pm}\left(\zeta^{ \pm}, \log y_{1}^{ \pm}\right)+\cdots, \quad x \in \Omega_{R}^{ \pm}, \\
g^{0}(x) & =r^{\lambda-1} \mathcal{G}^{0 \pm}(\log r)+\cdots, \quad x \in \Gamma_{R}^{0 \pm}=\{x: r>R, \varphi= \pm \alpha\} \\
g^{ \pm}(x) & =\left(y_{1}^{ \pm}\right)^{\lambda-1} \mathcal{G}^{1 \pm}\left(\log y_{1}^{ \pm}\right)+\cdots, \quad x \in \Gamma_{R}^{1 \pm}=\left\{x: r>R, y_{2}^{ \pm}=-a_{ \pm}\left(y_{1}^{ \pm}\right)^{s}\right\} .
\end{aligned}
$$

The functions on the right in $(3.20){ }_{1-4}$ depend polynomially on logarithms and smoothly on $\varphi \in[-\alpha, \alpha]$ and $\zeta^{ \pm} \in\left[-a_{ \pm}, 0\right]$.

As earlier in 3.1, we substitute the Ansatzen (3.19) and (3.20) in (1.13) and separate the principal asymptotic terms to formulate the problems for determining the coefficients $\Phi$ and $\mathcal{V}^{ \pm}$. Considering the system of equations in the region $\Omega_{R}^{0}$, we come to formula (3.3). Inside the parabolic sets $\Omega_{R}^{ \pm}$, the operator $\mathbf{L}^{ \pm}\left(\nabla_{y^{ \pm}}\right)$obtained from the operator $\mathcal{D}\left(\nabla_{x}\right)^{*} \mathbf{A}^{ \pm} \mathcal{D}\left(\nabla_{x}\right)$ by passing to local Cartesian coordinates $y^{ \pm}$(see the assumption (1.16) and compare with formulas (2.58) $)$ and written using the variables $\left(y_{1}^{ \pm}, \zeta^{ \pm}\right)$admits the separation

$$
L^{ \pm}\left(\nabla_{y^{ \pm}}\right)=\left(y_{1}^{ \pm}\right)^{-2 s} \mathcal{D}^{ \pm}\left(0, \partial_{\zeta^{ \pm}}\right)^{*} \mathbf{A}^{ \pm} \mathcal{D}^{ \pm}\left(0, \partial_{\zeta^{ \pm}}\right)+\cdots .
$$

This means that the coefficient $\mathcal{V}^{ \pm}$on the right-hand side $(3.19)_{2}$ must satisfy the following system of ordinary differential equations with parameter $l=\log y_{1}^{ \pm}$:

$$
\mathcal{D}^{ \pm}\left(0, \partial_{\zeta^{ \pm}}\right)^{*} \mathbf{A}^{ \pm} \mathcal{D}^{ \pm}\left(0, \partial_{\zeta^{ \pm}}\right) \mathcal{V}^{ \pm}\left(\zeta^{ \pm}, l\right)=\mathcal{F}^{ \pm}\left(\zeta^{ \pm}, l\right), \quad \zeta^{ \pm} \in\left(-a_{ \pm}, 0\right) .
$$

On the $\operatorname{arcs} \Gamma_{R}^{ \pm}$, the projections $\mathfrak{n}_{i}^{ \pm}\left(y^{ \pm}\right)$of the normal $\nu^{ \pm}(x)$ to the axes $y_{i}^{ \pm}$have the form

$$
\mathfrak{n}_{1}^{ \pm}\left(y_{1}^{ \pm}\right)=-\left(1+\mathfrak{n}^{ \pm}\left(y_{1}^{ \pm}\right)^{2}\right)^{-1 / 2} \mathfrak{n}^{ \pm}\left(y_{1}^{ \pm}\right), \quad \mathfrak{n}_{2}^{ \pm}\left(y_{1}^{ \pm}\right)=-\left(1+\mathfrak{n}^{ \pm}\left(y_{1}^{ \pm}\right)^{2}\right)^{-1 / 2}
$$


Also, due to the inclusion $s \in(0,1)$, the function $\mathfrak{n}^{ \pm}\left(y^{ \pm}\right)=a_{ \pm} s\left(y_{1}^{ \pm}\right)^{s-1}$ is infinitesimally small as $y_{1}^{ \pm} \rightarrow+\infty$. Therefore, the main term of the separated operator of the Neumann boundary conditions on $\Gamma_{R}^{ \pm}$,

$$
N^{ \pm}\left(x, \nabla_{x}\right)=\left(y_{1}^{ \pm}\right)^{-s}{\overline{\mathcal{D}^{ \pm}(0,-1)}}^{\top} \mathbf{A}^{ \pm} \mathcal{D}^{ \pm}\left(0, \partial_{\zeta^{ \pm}}\right)+\cdots
$$

implies the following boundary condition for the system (3.21):

$$
{\overline{\mathcal{D}^{ \pm}}(0,-1)}^{\top} \mathbf{A}^{ \pm} \mathcal{D}^{ \pm}\left(0, \partial_{\zeta^{ \pm}}\right) \mathcal{V}^{ \pm}\left(-a_{ \pm}, l\right)=\mathcal{G}^{1 \pm}(l) .
$$

The boundary condition at the point $\zeta^{ \pm}=0$ is derived from the second matching condition in $(1.13)_{2}$ with an additional summand $(\underline{3.20})_{3}$ on the right-hand side:

$$
{\overline{\mathcal{D}^{ \pm}(0,1)}}^{\top} \mathbf{A}^{ \pm} \mathcal{D}^{ \pm}\left(0, \partial_{\zeta^{ \pm}}\right) \mathcal{V}^{ \pm}(0, l)=\mathcal{G}^{0 \pm}(l)+\left.r^{1-\lambda} \mathbf{N}^{ \pm}\left(\nabla_{x}\right) r^{\lambda} \Phi(\varphi, \log r)\right|_{\substack{\varphi= \pm \alpha \\ \log r=l}} .
$$

We recall that according to the convention adopted in 1.2, on the left-hand side of (3.25) we use the inward (with respect to $\mathbb{K}$ ) normal to the side of the sector, and on the right-hand side we use the outward normal.

The first matching conditions in $(1.13)_{2}$ will be fully satisfied if we set

$$
\mathcal{V}^{ \pm}(0, l)=0 .
$$

The Neumann problem (3.21), (3.24), (3.25) admits the following additional requirement for a solution, which determines the solution uniquely: the corresponding homogeneous problem is satisfied by constant columns and only them. Since the problem is formally self-adjoint, the Fredholm alternative provides the following solvability conditions:

$$
\Psi^{ \pm}(l):=-\int_{-a_{ \pm}}^{0} \mathcal{F}^{ \pm}( \pm \zeta, l) d \zeta-\mathcal{G}^{1 \pm}(l)-\mathcal{G}^{0 \pm}(l)=\left.r^{1-\lambda} \mathbf{N}^{ \pm}\left(\nabla_{x}\right) r^{\lambda} \Phi(\varphi, \log r)\right|_{\substack{\varphi= \pm \alpha \\ \log r=l}}
$$

which become the boundary conditions (3.10) on the sides of $\mathbb{K}$.

Thus, having found a particular solution $r^{\lambda} \Phi(\varphi, \log r)$ of the model problem (3.3), (3.10), we obtain the vector-valued functions $\mathcal{V}^{ \pm}$from relations (3.21), (3.24)-(3.26). All terms of the Ansatz (3.19) are constructed.

Remark 3.1. If all terms singled out on the right-hand sides of (3.20) vanish, then $r^{\lambda} \Phi$ is a polynomial-logarithmic solution (2.5) of the homogeneous problem (2.1), and the solutions $\mathcal{V}^{ \pm}$of the problems (3.21), (3.24)-(3.26) become zero solutions. Therefore, extending the solutions $U_{(q)}^{j, p}(x)=r^{\lambda_{q}} \Phi(\varphi, \log r)$ to parabolic sets (1.3) by using expressions $y^{ \pm} \mapsto\left(y_{1}^{ \pm}\right)^{\lambda_{q}} \Phi( \pm \alpha / 2, \log r)$ independent of the transverse coordinate $y_{2}^{ \pm}$, generates mismatches in the homogeneous problem (1.13), which vanish at infinity sufficiently fast (cf. 3.2, where similar constructions were discussed for a sector with periodic boundary).

\section{Proof of ASYMPtotic EXPANSION \\ IN THE CASE OF A REGION WITH PERIODIC BOUNDARY}

4.1. Main theorem. This section contains the proof (divided into several steps) of the following theorem.

Theorem 4.1. Let $l \in \mathbb{N}, \gamma \in(l-1 / 2, l)$, and $\beta^{1}, \beta^{2} \in \mathbb{R}$ with $\beta^{1}<\beta^{2}$, and suppose there are no eigenvalues of the pencil (2.4) on the lines $\Lambda_{l-\beta^{1}}, \Lambda_{l-\beta^{2}}$. We also assume that the difference $l-\gamma>0$ is small and

$$
\beta^{2}-\beta^{1} \leq \delta_{\beta}<1 \text {. }
$$

Finally, choose an arbitrary number $\beta^{0} \in\left(\beta^{1}, \beta^{2}\right)$ such that the line $\Lambda_{l-\beta^{0}}$ also does not contain points of the spectrum of the pencil (2.4). A solution $u \in \mathfrak{V}_{\beta^{1}, \gamma}^{l+1,0}(G)^{k}$ of the 
problem (1.10) with the right-hand side $\{f, g\} \in \mathfrak{V}_{\beta^{2}, \gamma}^{l-1}(G)^{k} \times \mathfrak{V}_{\beta^{2}, \gamma}^{l-1 / 2}(\partial G)^{k}$ admits an asymptotic expansion

$$
u(x)=\chi_{\infty}(r) \sum c_{(q)}^{j, p} \mathcal{U}_{(q)}^{j, p}+\widetilde{u}(x),
$$

where $\chi_{\infty}$ is a cut-off function introduced before formula (3.14), $\mathcal{U}_{(q)}^{j, p}$ is the asymptotic block (3.17) containing polynomial-logarithmic solutions (2.5), and the summation is over all eigenvalues of the pencil (2.4) between the lines $\Lambda_{l-\beta^{0}}$ and $\Lambda_{l-\beta^{1}}$. The coefficients $c_{(q)}^{j, p} \in \mathbb{C}$ and the remainder $\widetilde{u}$ belonging to the space $\mathfrak{V}_{\beta^{0}, \gamma}^{l+1,0}(G)^{k}$ satisfy the inequality

$$
\begin{aligned}
& \left\|\widetilde{u} ; \mathfrak{V}_{\beta^{0}, \gamma}^{l+1,0}(G)\right\|+\sum\left|c_{(q)}^{j, p}\right| \\
& \quad \leq c\left(\left\|f ; \mathfrak{V}_{\beta^{2}, \gamma}^{l-1}(G)\right\|+\left\|g ; \mathfrak{V}_{\beta^{2}, \gamma}^{l-1 / 2}(\partial G)\right\|+\left\|u ; \mathfrak{V}_{\beta^{1}, \gamma}^{l+1,0}(G)\right\|\right) .
\end{aligned}
$$

The statement of Theorem 4.1 differs from the statement of Theorem 2.2 and Proposition 2.1, 2): Theorem 4.1] does not guarantee that the remainder $\widetilde{u}$ in the representation (4.2) belongs to the space $\mathfrak{V}_{\beta^{2}, \gamma}^{l+1,0}(G)^{k}$ with the same weight index $\beta^{2}$ as on the right-hand sides. The decrease of the index is caused by the method of reducing the original problem to the model problem in the sector $\mathbb{K}$ and of determining the properties of the asymptotic remainder near the periodic boundary. However, this does not prevent us from using the theorem to establish partial or complete asymptotic expansions of solutions constructed using the procedure described in 3.1. Moreover, since for $s>l$ and $\mu-s>\beta-l$ the space $\mathfrak{V}_{\mu, \gamma}^{s+1,0}(G)^{k}$ can be completely imbedded in the space $\mathfrak{V}_{\beta, \gamma}^{l+1,0}(G)^{k}$, Theorem 4.1 together with Proposition 4.2 below establishes that the kernel of the operator 3 (1.30) of the problem (1.10) is finite-dimensional.

Note that it suffices to establish Theorem 4.1 for small $\delta_{\beta}$ in (4.2): applying this result several times and increasing the index $\beta_{0}$ each time, we prove the full version of Theorem 4.1 (see Remark 4.2 below). In what follows, we assume that $\delta_{\beta}>0$ is sufficiently small but, of course, does not depend on $\beta^{1}$.

4.2. Auxiliary constructions. Cover the region $G$ by standard sets $\xi^{\vartheta}$ and $\Xi^{\vartheta}$ of several kinds; here $\xi^{\vartheta} \subset \Xi^{\vartheta}$ and $\partial \xi^{\vartheta} \cap \overline{\Xi^{\vartheta}} \subset \partial G$, and $\vartheta$ runs over all various indices introduced below. First, we cut off the bounded parts $\xi^{c}=G \cap \mathbb{B}_{2 R}$ and $\Xi^{c}=G \cap \mathbb{B}_{3 R}$ of $G$. Also, in the sector $\mathbb{K}_{R}\left(\alpha_{i}\right)$ we take the sets

$$
\begin{aligned}
\xi^{\alpha m} & =\left\{x: 2^{m-3 / 4}<R^{-1} r<2^{m+3 / 4},|\varphi|<\alpha_{1}\right\}, \\
\Xi^{\alpha m} & =\left\{x: 2^{m-1}<R^{-1} r<2^{m+1},|\varphi|<\alpha_{2}\right\},
\end{aligned}
$$

where $m \in \mathbb{N}$ and $\alpha_{i} \in(0, \alpha)$ will be chosen later; clearly, $\alpha_{1}<\alpha_{2}<\alpha$. The sets adjacent to the periodic curves $\Gamma_{R}^{ \pm}$are defined as follows:

$$
\begin{aligned}
\xi^{j \pm} & =\left\{x: y_{1}^{ \pm}-2 R-j \in(-3 / 4,3 / 4), 2 H^{0}>y_{2}^{ \pm}>H^{ \pm}\left(y_{1}^{ \pm}\right)\right\}, \\
\Xi^{j \pm} & =\left\{x: y_{1}^{ \pm}-2 R-j \in(-1,1), 3 H^{0}>y_{2}^{ \pm}>H^{ \pm}\left(y_{1}^{ \pm}\right)\right\} .
\end{aligned}
$$

The size of these sets does not depend on $j \in \mathbb{N}$, and the size along the axis $y_{1}^{ \pm}$is measured in terms of the (unit) period of the functions $H^{ \pm}$; in addition, we assume that $R>1$ and $H^{0}>\max \left\{H^{ \pm}\left(y_{1}\right) \mid y_{1} \in[0,1]\right\}$. The size of the sets (4.4) is proportional to $2^{m}$ and increases as $m$ grows. Introduce the rectangles $\xi^{m j \pm}$ and $\Xi^{m j \pm}$ that also have the size $O\left(2^{m}\right)$ and cover the regions $\Sigma^{ \pm}$bordered by the rays $\left\{x: y_{1}^{ \pm}>R, y_{2}^{ \pm}=2 H^{0}\right\}$, $\left\{x: r>R, \varphi= \pm \alpha_{1}\right\}$ and the circle $\partial \mathbb{B}_{R}$. Small rectangles $\xi^{m j \pm}$ are lined up in the

\footnotetext{
${ }^{3}$ Passage to the adjoint operator and increase of smoothness establish that the operator (1.30 is Fredholm with a finite-dimensional cokernel provided the condition (1.31) is satisfied and the pencil (2.4) has no eigenvalues on the line $\Lambda_{l-\beta}$ (cf. arguments in [37]).
} 
strips $\left\{x: 2^{m+3 / 4} H^{0}>y_{2}^{ \pm}>2^{m-3 / 4} H^{0}\right\}$; the size along the axis $y_{1}^{ \pm}$is $b H^{0} 2^{m}$ and the distance between the centers of neighboring rectangles is $3 b H^{0} 2^{m-2}$, so that they overlap. The larger rectangle $\Xi^{m j \pm}$ is obtained from the smaller $\xi^{m j \pm}$ using homothety with coefficient $4 / 3$ from the common center of these rectangles. The sets $\xi^{m j \pm}$ that are disjoint from $\Sigma^{ \pm}$are not considered, and the number $j \in \mathbb{N}$ increases as the distance from the axis $y_{2}^{ \pm}$increases. The factor $b>0$ and the angles $\alpha_{1}, \alpha_{2}$ can always be taken so that each of the families $\left\{\xi^{\vartheta}\right\}$ and $\left\{\Xi^{\vartheta}\right\}$ be a finite-multiplicity covering of the region $G$.

These complicated constructions are needed to ensure special properties of coverings. If $\xi^{\vartheta}, \Xi^{\vartheta}$ is one of the pairs of constructed sets, then on each of these sets, the following relations hold:

$$
\begin{gathered}
0<c_{1} \leq \delta_{\Xi^{\vartheta}}^{-1} \rho(x) \leq c_{2}, \\
0<C_{1} \leq(1+|x|)^{-1}\left(1+\left|x_{\Xi^{\vartheta}}\right|\right) \leq C_{2} .
\end{gathered}
$$

Here $\delta_{\Xi^{\vartheta}}$ and $x_{\Xi^{\vartheta}}$ are the diameter and the center of mass of $\Xi^{\vartheta}$, and the constants $c_{i}$ and $C_{i}$ can be taken the same for all elements of the covers. The change of coordinates

$$
x \mapsto \eta^{\vartheta \bullet}=\delta_{\Xi^{\vartheta}}^{-1}\left(x-x_{\Xi^{\vartheta}}\right)
$$

transforms the sets $\xi^{\vartheta}$ and $\Xi^{\vartheta}$ into the standard sets $\xi^{\vartheta \bullet}$ into $\Xi^{\vartheta \bullet}$ of unit size; among these sets are sectors of an annulus (see formulas (4.4)), trapezoids with curved bases (see formulas (4.5) ), rectangles, and compressed sets $\xi^{c}$ and $\Xi^{c}$. In coordinates $\eta$ the system of equations and homogeneous boundary conditions in the problem (1.10) restricted to the region $\Xi_{G}^{\vartheta}=\Xi^{\vartheta} \cap G$ and the arc $\Xi^{\vartheta} \cap \partial G$ respectively, take the following form:

$$
\begin{gathered}
L\left(\nabla_{\eta^{\vartheta}}\right) u^{\vartheta \bullet}\left(\eta^{\vartheta \bullet}\right)=\delta_{\Xi^{\vartheta}}^{2} f^{\vartheta \bullet}\left(\eta^{\vartheta \bullet}\right), \quad \eta^{\vartheta \bullet} \in \Xi_{G}^{\vartheta \bullet} ; \\
N\left(\eta^{\vartheta \bullet}, \nabla_{\eta^{\vartheta \bullet}}\right) u^{\vartheta \bullet}\left(\eta^{\vartheta \bullet}\right)=0, \quad \eta^{\vartheta \bullet} \in\left(\Xi^{\vartheta} \cap \partial G\right)^{\bullet},
\end{gathered}
$$

where $u^{\vartheta \bullet}$ and $f^{\vartheta \bullet}$ are vector-valued functions $u$ and $f$ written using the variable $\eta^{\vartheta \bullet}$. Denote by $\bar{u}^{\vartheta}$ the average of the vector-valued function $u$ over the region $\Xi_{G}^{\vartheta}$. Due to the structure of the operators (1.5) and (1.11) the difference $u^{\vartheta \bullet}-\bar{u}^{\vartheta}$ satisfies the same problem (4.7). By the Poincaré inequality

$$
\left\|u^{\vartheta \bullet}-\bar{u}^{\vartheta} ; L_{2}\left(\Xi_{G}^{\vartheta \bullet}\right)\right\| \leq c\left\|\nabla_{\eta^{\vartheta} \bullet}\left(u^{\vartheta \bullet}-\bar{u}^{\vartheta}\right) ; L_{2}\left(\Xi_{G}^{\vartheta \bullet}\right)\right\|=c\left\|\nabla_{\eta^{\vartheta} \bullet} u^{\vartheta \bullet} ; L_{2}\left(\Xi_{G}^{\vartheta \bullet}\right)\right\|,
$$

the local estimate of a solution of an elliptic system (see [38, 39]) can be written as follows:

$$
\begin{aligned}
\left\|\nabla_{\eta^{\vartheta \bullet}} u^{\vartheta \bullet} ; H^{l}\left(\xi_{G}^{\vartheta \bullet}\right)\right\|^{2} & \leq\left\|u^{\vartheta \bullet}-\bar{u}^{\vartheta} ; H^{l+1}\left(\xi_{G}^{\vartheta \bullet}\right)\right\|^{2} \\
& \leq c\left(\delta_{\Xi^{\vartheta}}^{4}\left\|f^{\vartheta \bullet} ; H^{l-1}\left(\Xi_{G}^{\vartheta \bullet}\right)\right\|^{2}+\left\|u^{\vartheta \bullet}-\bar{u}^{\vartheta} ; L_{2}\left(\Xi_{G}^{\vartheta \bullet}\right)\right\|^{2}\right) \\
& \leq c\left(\delta_{\Xi_{\vartheta} \vartheta}^{4}\left\|f^{\vartheta \bullet} ; H^{l-1}\left(\Xi_{G}^{\vartheta \bullet}\right)\right\|^{2}+\left\|\nabla_{\eta^{\vartheta \bullet}} u^{\vartheta \bullet} ; L_{2}\left(\Xi_{G}^{\vartheta \bullet}\right)\right\|^{2}\right) .
\end{aligned}
$$

Returning to coordinates $x$ and multiplying (4.8) by $\left(1+\left|x_{\Xi^{\vartheta}}\right|\right)^{2(\beta-\gamma)} \delta_{\Xi^{\vartheta}}^{2(\gamma-l-1)-1}$, we transform inequality (4.8) without its middle part into the following:

$$
\begin{aligned}
& \sum_{j=1}^{l+1}\left(1+\left|x_{\Xi^{\vartheta}}\right|\right)^{2(\beta-\gamma)} \delta_{\Xi^{\vartheta}}^{2(\gamma-l-1+j)}\left\|\nabla_{x}^{j} u ; L_{2}\left(\xi_{G}^{\vartheta}\right)\right\|^{2} \\
& \leq c\left(\sum_{h=0}^{l-1}\left(1+\left|\xi_{\Xi^{\vartheta}}\right|\right)^{2(\beta-\gamma)} \delta_{\Xi^{\vartheta}}^{2(\gamma-l+1+h)}\left\|\nabla_{x}^{h} f ; L_{2}\left(\Xi_{G}^{\vartheta}\right)\right\|^{2}\right. \\
& \left.+\left(1+\left|x_{\Xi^{\vartheta}}\right|\right)^{2(\beta-\gamma)} \delta_{\Xi^{\vartheta}}^{2(\gamma-l)}\left\|\nabla_{x} u ; L_{2}\left(\Xi_{G}^{\vartheta}\right)\right\|^{2}\right) .
\end{aligned}
$$


Due to relations (4.6), weight factors can be moved inside the $L_{2}$-norms of vectorvalued functions $u, f$, and their derivatives. Taking into account all these changes we get the following inequality:

$$
\begin{aligned}
& \sum_{j=1}^{l+1} \|(1+r)^{\beta-\gamma} \rho^{\gamma-l-1+j} \nabla_{x}^{j} u ; L_{2}\left(\xi_{G}^{\vartheta}\right) \|^{2} \\
& \leq c\left(\sum_{h=0}^{l-1}\left\|(1+r)^{\beta-\gamma} \rho^{\gamma-l+1+h} \nabla_{x}^{h} f ; L_{2}\left(\Xi_{G}^{\vartheta}\right)\right\|^{2}\right. \\
&\left.+\left\|(1+r)^{\beta-\gamma} \rho^{\gamma-l} \nabla_{x} u ; L_{2}\left(\Xi_{G}^{\vartheta}\right)\right\|^{2}\right) .
\end{aligned}
$$

Since the local estimate was used for a finite number of "model" regions and the operator $L$ has constant coefficients outside the disc $\mathbb{B}_{R}$, the factor $c$ in (4.9) can be taken the same for all elements of the covering. Let us sum up over all elements $\xi^{\vartheta}$ and recall the definition of the norm (1.20). Since the covering has finite multiplicity, we obtain the following inequality:

$$
\left\|\nabla_{x} u ; \mathfrak{V}_{\beta, \gamma}^{l}(G)\right\|^{2} \leq c\left(\left\|f ; \mathfrak{V}_{\beta, \gamma}^{l-1}(G)\right\|^{2}+\left\|g ; \mathfrak{V}_{\beta, \gamma}^{l-1 / 2}(\partial G)\right\|^{2}+\left\|\nabla_{x} u ; \mathfrak{V}_{\beta-l, \gamma-l}^{0}(G)\right\|^{2}\right),
$$

where the vector-valued function $g$ in the boundary condition in the problem (1.10) is so far assumed to be equal to zero. Let us emphasize that the finiteness of the right-hand side of (4.10) guarantees the finiteness of the left-hand side.

Proposition 4.1. Let $u \in \mathfrak{V}_{\beta-l, \gamma-l}^{1,0}(G)^{k}$ be a solution of the problem (1.10) and

$$
f \in \mathfrak{V}_{\beta, \gamma}^{l-1}(G)^{k}, \quad g \in \mathfrak{V}_{\beta, \gamma}^{l-1 / 2}(\partial G)^{k}
$$

for some $l \in \mathbb{N}$ and $\beta, \gamma \in \mathbb{R}$. Then $u \in \mathfrak{V}_{\beta, \gamma}^{l+1,0}(G)^{k}$ and the estimate (4.10) holds.

Proof. We can make boundary conditions homogeneous by substracting from a solution $u$ the vector-valued function $v \in \mathfrak{V}_{\beta, \gamma}^{l+1}(G)^{k} \subset \mathfrak{V}_{\beta, \gamma}^{l+1,0}(G)^{k}$ determined by Lemma 1.1 and satisfying the relations (1.25) and (1.26).

The next result (both the formulation and the proof) establishes the existence of a quasiparametrix $\mathfrak{R}$, i.e., a continuous linear mapping

$$
\mathfrak{R}: \mathfrak{V}_{\beta, \gamma}^{l-1}(G)^{k} \times \mathfrak{V}_{\beta, \gamma}^{l-1 / 2}(\partial G)^{k} \rightarrow \mathfrak{V}_{\beta, \gamma}^{l+1}(G)^{k}
$$

such that the operator

$$
\{L, N\} \mathfrak{R}-\mathbb{I}: \mathfrak{V}_{\beta, \gamma}^{l-1}(G)^{k} \times \mathfrak{V}_{\beta, \gamma}^{l-1 / 2}(\partial G)^{k} \rightarrow \mathfrak{V}_{\beta+1, \gamma+1}^{l}(G)^{k} \times \mathfrak{V}_{\beta+1, \gamma+1}^{l+1 / 2}(\partial G)^{k}
$$

is continuous; here $\mathbb{I}$ is the identity map. We emphasize that the operator (4.12) cannot be a genuine parametrix for the operator (1.27) of the problem (1.10) since the imbedding of the right direct product of function spaces into the left one in formula (4.13) is not a compact operator.

Proposition 4.2. For each continuous vector-valued functions (4.11) there exists an element $u^{\{f, g\}}$ of the space $\mathfrak{V}_{\beta, \gamma}^{l+1}(G)^{k}$ satisfying the inequality

$$
\begin{aligned}
\left\|u^{\{f, g\}} ; \mathfrak{V}_{\beta, \gamma}^{l+1}(G)\right\|+\left\|L u^{\{f, g\}}-f ; \mathfrak{V}_{\beta+1, \gamma+1}^{l}(G)\right\| \\
\quad+\left\|N u^{\{f, g\}}-g ; \mathfrak{V}_{\beta+1, \gamma+1}^{l+1 / 2}(\partial G)\right\| \\
\leq c\left(\left\|f ; \mathfrak{V}_{\beta, \gamma}^{l-1}(G)\right\|+\left\|g ; \mathfrak{V}_{\beta, \gamma}^{l-1 / 2}(\partial G)\right\|\right) .
\end{aligned}
$$


Proof. First of all, note that the boundary conditions in the problem (1.10) can be made homogeneous by using the vector-valued function $v \in \mathfrak{V}_{\beta, \gamma}^{l+1}(G)^{k}$ from Lemma 1.1, 2). By the construction of the coverings $\left\{\xi^{\vartheta}\right\}\left\{\Xi^{\vartheta}\right\}$ there exist functions $\chi^{\vartheta} \in C_{c}^{\infty}\left(\xi^{\vartheta}\right)$ and $X^{\vartheta} \in C_{c}^{\infty}\left(\Xi^{\vartheta}\right)$ such that

$$
\chi^{\vartheta} X^{\vartheta}=\chi^{\vartheta} ; \quad\left|\nabla_{x}^{p} \chi^{\vartheta}(x)\right|+\left|\nabla_{x}^{p} X^{\vartheta}(x)\right| \leq c_{p} \delta_{\Xi \vartheta}^{-p}, \quad p \in \mathbb{N}_{0} ; \quad \sum_{\vartheta} \chi^{\vartheta}=1,
$$

for each element of the covering (the summation is over all indices $\vartheta$ ).

Let $\chi^{\vartheta}$ be one of the elements of the partition of unity supported on the set $\xi^{\vartheta}$. We pass to coordinates $\eta^{\vartheta \bullet}$ and consider the system of differential equations (4.7) with homogeneous Neumann boundary conditions. Since this system is elliptic, general theory (see [38, 39]) yield a vector-valued function $w^{\vartheta} \in H^{l+1}\left(\Xi_{G}^{\vartheta \bullet}\right)^{k}$ such that

$$
\begin{aligned}
\left\|X^{\vartheta \bullet} w^{\vartheta} ; H^{l+1}\left(\Xi_{G}^{\vartheta \bullet}\right)\right\|^{2}+\left\|L X^{\vartheta \bullet} w^{\vartheta}-\delta_{\Xi^{\vartheta}}^{2} \chi^{\vartheta \bullet} f^{\vartheta \bullet} ; H^{l}\left(\Xi_{G}^{\vartheta \bullet}\right)\right\|^{2} \\
\quad+\left\|N X^{\vartheta \bullet} w^{\vartheta} ; H^{l+1 / 2}\left(\partial \Xi^{\vartheta} \cap \partial G\right)^{\bullet}\right\|^{2} \\
\leq c \delta_{\Xi^{\vartheta}}^{4}\left\|\chi^{\vartheta \bullet} f^{\vartheta \bullet} ; H^{l-1}\left(\Xi_{G}^{\vartheta \bullet}\right)\right\|^{2},
\end{aligned}
$$

where, as before, the same constant $c$ can be taken for all elements of the covering (of course, this is true for the constants $c_{p}$ in (4.15) as well). The desired vector-valued function $u^{\{f, 0\}}$ equals the sum of products $X^{\vartheta} w^{\vartheta}$ constructed for each index $\vartheta$. Using the same computations that allowed us to transform the estimate (4.8) into inequality (4.10), taking into account relations (4.15), and we can obtain formula (4.14) simplified in the case of $g=0$ by processing and adding together inequalities (4.16). Certain differences that occur in considering the last summand in (4.16) are discussed in the next remark.

Remark 4.1. If the support of the function $z \in \mathfrak{V}_{\beta, \gamma}^{l}(G)$ lies in the $d$-neighborhood of the boundary $\partial G$ where, according to definition (1.21), we have $1 \leq \rho(x) \leq c(d)$, then the norm (1.20) is equivalent to the Sobolev norm $\left\|\left(1+r^{2}\right)^{(\beta-\gamma) / 2} z ; H^{l}(G)\right\|$ (cf. Lemma 1.1. 1)). Therefore, for $l \in \mathbb{N}$ the trace of the function $z$ on the curve $\partial G$ exists and is characterized by the norm $\left\|\left(1+r^{2}\right)^{(\beta-\gamma) / 2} z ; H^{l-1 / 2}(\partial G)\right\|$. The norm in the SobolevSlobodetskii space $H^{l-1 / 2}(\partial G)$ can be defined by the formula

$$
\begin{aligned}
\left\|Z ; H^{l-1 / 2}(\partial G)\right\|= & \sum_{j=0}^{l-1}\left\|\partial_{s}^{j} Z ; L_{2}(\partial G)\right\| \\
& +\left(\int_{\partial G} \int_{\{\sigma \in \partial G:|s-\sigma|<d\}}\left|\partial_{s}^{l-1} Z(s)-\partial_{\sigma}^{l-1} Z(\sigma)\right|^{2} \frac{d s d \sigma}{|s-\sigma|^{2}}\right)^{1 / 2},
\end{aligned}
$$

where $d$ is a positive constant (it can be chosen arbitrarily), $s$ is the arc length, and $|s-\sigma|$ is the distance between the points with coordinates $s$ and $\sigma$ along the line $\partial G$. Using formula (4.17) and the middle inequality in (4.15), it is clear that the following norms (which arose in the verification of Proposition 4.2) are equivalent:

$$
\begin{aligned}
&\left(\sum_{\vartheta} \|(1\right.\left.\left.+r^{2}\right)^{(\beta-\gamma) / 2} N X^{\vartheta} w^{\vartheta} ; H^{l+1 / 2}\left(\partial \Xi^{\vartheta} \cap \partial G\right) \|^{2}\right)^{1 / 2} \\
& \sim\left\|\left(1+r^{2}\right)^{(\beta-\gamma) / 2} N \sum_{\vartheta} X^{\vartheta} w^{\vartheta} ; H^{l+1 / 2}(\partial G)\right\| .
\end{aligned}
$$

Notice also that since the principal part of the operator

$$
u \mapsto N^{\beta-\gamma}\left(x, \nabla_{x}\right) u(x)=\left(1+r^{2}\right)^{(\gamma-\beta) / 2} N\left(x, \nabla_{x}\right)\left(\left(1+r^{2}\right)^{(\beta-\gamma) / 2} u(x)\right)
$$


is the same as the principal part of the operator (1.11) of the Neumann boundary conditions, the pair $\left\{\mathbb{I}, N^{\beta-\gamma}\right\}$ is a Dirichlet system on $\partial G$, and Lemma 1.1, 2) follows, for example, from the results of [40, Chapter 2].

4.3. Reduction to the model problem in the sector. Let $u \in \mathfrak{V}_{\beta^{1}, \gamma}^{l+1,0}(G)^{k}$ be a solution of the problem (1.10) with right-hand sides satisfying (4.11), where $\beta=\beta^{2}$ and the indices $\beta^{1}, \beta^{2}$ and $\gamma, l$ satisfy the hypotheses of Theorem 4.1. Also, let $u^{\{f, g\}} \in$ $\mathfrak{V}_{\beta^{2}, \gamma}^{l+1}(G)^{k}$ be a vector-valued function obtained using Proposition 4.2, By (4.14), the difference $u^{1}=u-u^{\{f, g\}}$ satisfies the problem (1.10) with the new right-hand sides

$$
f^{1} \in \mathfrak{V}_{\beta^{2}+1, \gamma+1}^{l}(G)^{k}, \quad g^{1} \in \mathfrak{V}_{\beta^{2}+1, \gamma+1}^{l+1 / 2}(\partial G)^{k} .
$$

Since $\beta^{2}>\beta^{1}$, inclusions (4.18) remain valid if we replace $\beta^{2}$ with $\beta^{1}$. Therefore, Proposition 4.1 implies that $u^{1} \in \mathfrak{V}_{\beta^{1}+1, \gamma+1}^{l+2,0}(G)^{k}$. Repeating this procedure, we obtain a solution $u^{2}$ of the same problem with the right-hand sides $f^{2}$ and $g^{2}$ belonging to the spaces in (4.18), with indices $\beta^{2}, \gamma$, and $l$ again increased by 1 . Also we have $u^{2} \in$ $\mathfrak{V}_{\beta^{1}+2, \gamma+2}^{l+3,0}(G)^{k}$. Repeating this procedure, we can increase the indices by an arbitrary given number $N \in \mathbb{N}$, and the norms of the new solutions and new right-hand sides will not exceed a constant multiplied by the sum of the old norms. Since in Theorem 4.1 we are allowed to include the term $u^{\{f, g\}} \in \mathfrak{V}_{\beta^{2}, \gamma}^{l+1}(G)^{k}$ and similar terms to the asymptotic remainder, we can assume from the very beginning that

$$
u \in \mathfrak{V}_{\beta^{1}+N, \gamma+N}^{l+1+N, 0}(G)^{k}, \quad f \in \mathfrak{V}_{\beta^{2}+N, \gamma+N}^{l-1+N}(G)^{k}, \quad g \in \mathfrak{V}_{\beta^{2}+N, \gamma+N}^{l+N-1 / 2}(\partial G)^{k} .
$$

Furthermore, multiplying the solution $u$ by a cut-off function $\chi_{\infty}$, we can ensure that the vector-valued functions $f=L u, g=N u$, and $u$ vanish inside the circle $\mathbb{B}_{2 R}$. None of these transformations violates the hypotheses of Theorem 4.1 .

We can assume without loss of generality (cf. Remark 1.1) that the sector $\mathbb{K}_{R}$ lies inside the set (1.1). Denote by $u^{ \pm}$the restriction of the solution $u$ to the sides $(\partial \mathbb{K})^{ \pm}$of the sector (we recall that these restrictions vanish for $r>2 R$ ). Since $\nabla_{x} u \in \mathfrak{V}_{\beta^{1}+N, \gamma+N}^{l+N}(G)^{k}$, Lemma 1.1, 1) and a simple imbedding theorem show that

$$
\begin{aligned}
\left(1+r^{2}\right)^{\left(\beta^{1}-\gamma\right) / 2} \frac{\partial u^{ \pm}}{\partial y_{1}^{ \pm}} & \in H^{l+N-1 / 2}\left((\partial \mathbb{K})^{ \pm}\right)^{k} \subset H^{l+N-1}\left((\partial \mathbb{K})^{ \pm}\right)^{k} \\
& \subset C^{l+N-2}\left((\partial \mathbb{K})^{ \pm}\right)^{k} .
\end{aligned}
$$

The norms of the derivative $\partial u^{ \pm} / \partial y_{1}^{ \pm}$in these spaces, hence also the same norms of the vector-valued function

$$
\mathbf{N}^{ \pm}\left(\nabla_{x}\right) u^{ \pm}={\overline{\mathcal{D}\left(\nu^{ \pm}\right)}}^{\top} \mathbf{A} \mathcal{D}^{ \pm}\left(\frac{\partial}{\partial y_{1}^{ \pm}}, 0\right) u^{ \pm}
$$

(cf. formulas (2.2) and (2.58)) are bounded from above by $c\left\|u ; \mathfrak{V}_{\beta^{1}+N, \gamma+N}^{l+1+N, 0}(G)\right\|$. Therefore, Lemma 1.1 2) shows that there exists a vector-valued function $v^{ \pm}$supported on the set

such that

$$
\left\{x \in \mathbb{K}: \operatorname{dist}\left(x,(\partial \mathbb{K})^{ \pm}\right) \leq d, r>3 R / 2\right\},
$$

$$
\begin{aligned}
\mathbf{N}^{ \pm}\left(\nabla_{x}\right) u^{ \pm} & =\mathbf{N}^{ \pm}\left(\nabla_{x}\right) v^{ \pm}(\partial \mathbb{K})^{ \pm} \\
\left\|\left(1+r^{2}\right)^{\left(\beta^{1}-\gamma\right) / 2} v^{ \pm} ; H^{l+1+N}(\mathbb{K})\right\| & \leq c\left\|v ; \mathfrak{V}_{\beta^{1}+N, \gamma+N}^{l+1+N}(G)\right\| \\
& \leq c\left\|u ; \mathfrak{V}_{\beta^{1}+N, \gamma+N}^{l+1+N, 0}(G)\right\| .
\end{aligned}
$$

The constants $c$ and the functions $v^{ \pm}$depend on the chosen size $d>0$. 
Consider the sum

$$
\mathfrak{U}(x)=\left(1-\chi_{d}(x)\right)\left(u(x)-\sum_{ \pm} \chi^{ \pm}(\varphi) u^{ \pm}\left(y_{1}^{ \pm}\right)\right)+\sum_{ \pm}\left(\chi^{ \pm}(\varphi) u^{ \pm}\left(y_{1}^{ \pm}\right)-v^{ \pm}(x)\right)
$$

where $\chi^{ \pm}$are cut-off functions used in (2.11), and the cut-off function $\chi_{d} \in C^{\infty}(\overline{\mathbb{K}})$ equals 1 in the $(d / 2)$-neighborhood $\mathcal{V}_{d / 2}$ of the boundary $\partial \mathbb{K}$ and vanishes outside the $d$ neighborhood $\mathcal{V}_{d}$. Using the first relation in (4.20), we obtain the equality $\mathbf{N}^{ \pm}\left(\nabla_{x}\right) \mathfrak{U}=0$ on $\partial \mathbb{K}$. Let us transform the summands in the expression

$$
\begin{aligned}
\mathfrak{F}= & \mathbf{L}\left(\nabla_{x}\right) \mathfrak{U} \\
= & \left(1-\chi_{d}\right) f-\left[\mathbf{L}\left(\nabla_{x}\right), \chi_{d}\right]\left(u-\sum_{ \pm} \chi^{ \pm} u^{ \pm}\right) \\
& -\sum_{ \pm} \mathbf{L}\left(\nabla_{x}\right) v^{ \pm}+\chi_{d} \sum_{ \pm} \mathbf{L}\left(\nabla_{x}\right) u^{ \pm}
\end{aligned}
$$

to verify that this expression belongs to the space $\mathfrak{\mathfrak { V }}_{\beta^{2}, \gamma^{2}}^{l-1}(\mathbb{K})^{k}$ with the norm defined by $(2.40)_{2}$. Here

$$
\gamma^{2}=\gamma+\beta^{2}-\beta^{1}
$$

and the number $\delta_{\beta}$ in (4.1) is chosen to be so small that both indices $\gamma$ and $\gamma^{2}$ satisfy (1.31).

By the definition of the cut-off function $\chi_{d}$, on the support $\operatorname{supp}\left(1-\chi_{d}\right)$ we have

$$
\begin{gathered}
\theta \geq c_{d}(1+r)^{-1}, \\
c_{d} \theta \leq(1+r)^{-1} \rho \leq C_{d} \theta .
\end{gathered}
$$

Therefore, formulas $(1.20)$ and $(2.40){ }_{2}$ for the weight norms, together with the condition $\gamma>\gamma^{2}$, yield

$$
\begin{aligned}
\left\|\left(1-\chi_{d}\right) f ;{\stackrel{\mathfrak{V}}{\beta^{2}, \gamma^{2}}}^{l-1}(\mathbb{K})\right\| & \leq c\left\|f ;{\stackrel{\mathfrak{V}}{\beta^{2}, \gamma^{2}}}^{l-1}\left(\mathbb{K} \backslash \mathcal{V}_{d / 2}\right)\right\| \\
& \leq c\left\|f ; \mathfrak{V}_{\beta^{2}, \gamma^{2}}^{l-1}(\mathbb{K})\right\| \leq c\left\|f ; \mathfrak{V}_{\beta^{2}, \gamma}^{l-1}(G)\right\| .
\end{aligned}
$$

The third and the fourth summands on the right-hand side of (4.22) are transformed using relations (4.19) and (4.20). Let $\xi^{m 0 \pm} \in \mathbb{K}$ be two rectangles of the same size such that their sides $\xi_{\#}^{m 0 \pm}$ are adjacent to the rays $\{x: r>R, \varphi= \pm \alpha\}$, respectively, and the rectangles themselves cover the supports of the vector-valued functions $\chi_{d} u^{ \pm}$and $v^{ \pm}$ (cf. the construction of covers in 4.2); here $m \in \mathbb{N}$. Also let $r_{m}$ be the distance between the vertex of the sector and the midpoint of the interval $\xi_{\#}^{m 0 \pm}$. For $x \in \xi^{m 0 \pm}$ we have the relations (similar to (4.6) $) c_{1} r \leq r_{m} \leq c_{2} r$ and $0 \leq \theta \leq c_{0} r_{m}^{-1}$ with positive constants $c_{j}$. Therefore, taking into account the bounds for the norms of the derivative $\partial u^{ \pm} / \partial y_{1}^{ \pm}$ 
for $N=2$ (mentioned after formula (4.19) ), we obtain the following chain of inequalities:

$$
\begin{aligned}
& \left\|\chi_{d} \mathbf{L}\left(\nabla_{x}\right) u^{ \pm} ; \mathfrak{\mathfrak { V }}_{\beta^{2}, \gamma^{2}}^{l-1}(\mathbb{K})\right\|^{2} \\
& \leq c \sum_{m=1}^{\infty} \sum_{p=0}^{l-1} \int_{\xi^{m 0 \pm}} r^{2\left(\beta^{2}-l+1+p\right)} \theta^{2\left(\gamma^{2}-l+1+p\right)} \sum_{q=2}^{p+2}\left|\frac{\partial^{q} u^{ \pm}}{\partial y_{1}^{q}}\left(y_{1}^{ \pm}\right)\right|^{2} d x \\
& \leq c \sum_{m=1}^{\infty} \sum_{p=0}^{l-1} r_{m}^{2\left(\beta^{2}-l+1+p\right)} r_{m}^{2\left(\gamma-\beta^{1}\right)} \int_{0}^{c_{0} / r_{m}} \theta^{2\left(\gamma^{2}-l+1+p\right)} r_{m} d \theta \\
& \quad \times\left\|\left(1+r^{2}\right)^{\left(\beta^{1}-\gamma\right) / 2} \frac{\partial^{2} u^{ \pm}}{\partial y_{1}^{2}} ; C^{l-1}\left(\xi_{\#}^{m 0 \pm}\right)\right\|^{2} \\
& \leq c \sum_{m=1}^{\infty} r_{m}^{2\left(\beta^{2}+\gamma-\beta^{1}-\gamma^{2}\right)}\left\|\left(1+r^{2}\right)^{\left(\beta^{1}-\gamma\right) / 2} \frac{\partial u^{ \pm}}{\partial y_{1}^{ \pm}} ; H^{l+1}\left(\xi_{\#}^{m 0 \pm}\right)\right\|^{2} \\
& \leq c\left\|\left(1+r^{2}\right)^{\left(\beta^{1}-\gamma\right) / 2} \frac{\partial u^{ \pm}}{\partial y_{1}^{ \pm}} ; H^{l+1}\left((\partial \mathbb{K})^{ \pm}\right)\right\|^{2} \leq c\left\|u ; \mathfrak{V}_{\beta^{1}+2, \gamma+2}^{l+3,0}(G)\right\| .
\end{aligned}
$$

We emphasize that the exponent $2\left(\beta^{2}+\gamma-\beta^{1}-\gamma^{2}\right)$ of the variable $r_{m}$ vanishes due to (4.23), and the integrals in the variable $\theta$ converge due to the restriction (1.31) for the index $\gamma^{2}$.

Similarly, using the estimate in (4.20) in the case $N=2$, we obtain the following chain of inequalities:

$$
\begin{aligned}
& \left\|\mathbf{L}\left(\nabla_{x}\right) v^{ \pm} ; \mathfrak{V}_{\beta^{2}, \gamma^{2}}^{l-1}(\mathbb{K})\right\|^{2} \\
& \leq c \sum_{m=1}^{\infty} \sum_{p=0}^{l-1} \int_{\xi^{m 0 \pm}} r^{2\left(\beta^{2}-l+1+p\right)} \theta^{2\left(\gamma^{2}-l+1+p\right)}\left|\nabla_{x}^{p+2} v^{ \pm}(x)\right|^{2} d x \\
& \leq c \sum_{m=1}^{\infty} \sum_{p=0}^{l-1} r_{m}^{2\left(\beta^{2}-l+1+p\right)} r_{m}^{2\left(\gamma-\beta^{1}\right)} \int_{0}^{c / r_{m}} \theta^{2\left(\gamma^{2}-l+1+p\right)} r_{m} d \theta \\
& \quad \times\left\|\left(1+r^{2}\right)^{\left(\beta^{1}-\gamma\right) / 2} v^{ \pm} ; C^{l+1}\left(\xi^{m 0 \pm}\right)\right\|^{2} \\
& \leq c \sum_{m=1}^{\infty} r_{m}^{2\left(\beta^{2}+\gamma-\beta^{1}-\gamma^{2}\right)}\left\|\left(1+r^{2}\right)^{\left(\beta^{1}-\gamma\right) / 2} v^{ \pm} ; H^{l+3}\left(\xi^{m 0 \pm}\right)\right\|^{2} \\
& \leq c\left\|\left(1+r^{2}\right)^{\left(\beta^{1}-\gamma\right) / 2} v^{ \pm} ; H^{l+3}(\mathbb{K})\right\|^{2} \leq c\left\|u ; \mathfrak{V}_{\beta^{1}+2, \gamma+2}^{l+3,0}(G)\right\| .
\end{aligned}
$$

To estimate the second summand on the right-hand side of (4.22) we need an auxiliary result. Note that this summand contains the commutator $\left[\mathbf{L}, \chi_{d}\right]=\mathbf{L} \chi_{d}-\chi_{d} \mathbf{L}$ and the supports of entries of a first order matrix differential operator belong to the set $\mathbb{K} \cap\left(\mathcal{V}_{d} \backslash \mathcal{V}_{d / 2}\right)$ where, according to (1.21), we have

$$
1 \leq \rho(x) \leq C, \quad 0<c \leq(1+r) \theta \leq C .
$$

Lemma 4.1. We have

$$
\left\|u-\sum_{ \pm} \chi^{ \pm} u^{ \pm} ; \mathfrak{V}_{\beta^{1}, \gamma}^{l+1}\left(\mathbb{K} \cap\left(\mathcal{V}_{d} \backslash \mathcal{V}_{d / 2}\right)\right)\right\| \leq c\left\|u ; \mathfrak{V}_{\beta^{1}, \gamma}^{l+1,0}(\mathbb{K})\right\| .
$$

Proof. Taking into account (4.26) and the above-mentioned facts concerning the norms of the vector-valued function (4.19), we conclude that it suffices to estimate the integral

$$
\int_{\mathbb{K} \cap\left(\mathcal{V}_{d} \backslash \mathcal{V}_{d / 2}\right)} r^{2\left(\beta^{1}-l-1\right)} \theta^{2(\gamma-l-1)}\left|u(x)-\sum_{ \pm} \chi^{ \pm}(\varphi) u^{ \pm}\left(y_{1}^{ \pm}\right)\right|^{2} d x,
$$


occurring, according to formula $(2.40)_{2}$, on the left-hand side of (4.27), in terms of the integral

$$
\int_{0}^{\infty}(1+r)^{2\left(\beta^{1}-\gamma\right)} r^{-1} \int_{-\alpha}^{\alpha}(1+(1+r \theta))^{2(\gamma-l)}\left|\frac{\partial u}{\partial \varphi}(x)\right|^{2} d \varphi d r
$$

occurring, in view of formula (1.29), on the right-hand side. Since the factor $1+$ $(1+r \theta)^{2(\gamma-l)}$ can be deleted from the integrand in (4.29) after reducing the domain of integration with respect to $\varphi$ to the union of intervals $\left.-\alpha,-\alpha+C(1+r)^{-1}\right)$ and $\left(\left(\alpha-C(1+r)^{-1}\right), \alpha\right)$, we obtain the required estimate from the following version of the Hardy inequality:

$$
\int_{0}^{\theta_{0}} \theta^{-2}|V(\theta)-V(0)|^{2} d \theta \leq 4 \int_{0}^{\theta_{0}}\left|\frac{d V}{d \theta}(\theta)\right|^{2} d \theta, \quad V \in H^{1}\left(0, \theta_{0}\right), \theta_{0}>0
$$

Indeed, this inequality implies that the integral in (4.29) bounds from above the sum

$$
c \sum_{ \pm} \pm \int_{0}^{\infty}(1+r)^{2\left(\beta^{1}-\gamma\right)-1} \int_{ \pm \alpha \mp C(1+r)^{-1}}^{ \pm \alpha} \theta^{-2}\left|u(x)-u^{ \pm}\left(y_{1}^{ \pm}\right)\right|^{2} d \varphi d r
$$

which, in turn, is larger that the integral (4.28) multiplied by a positive constant. We emphasize that inequalities (4.26) allow for a change of factors $\theta$ to factors $1+r$ and back, in formula (4.28).

Now we complete the verification of the fact that $\mathfrak{F} \in \mathfrak{\mathfrak { V }}_{\beta^{2}, \gamma^{2}}^{l-1}(G)^{k}$. We have

$$
\begin{aligned}
& \left\|\left[\mathbf{L}\left(\nabla_{x}\right), \chi_{d}\right]\left(u-\sum_{ \pm} \chi^{ \pm} u^{ \pm}\right) ; \mathfrak{V}_{\beta^{2}, \gamma^{2}}^{l-1}\left(\mathbb{K} \cap\left(\mathcal{V}_{d} \backslash \mathcal{V}_{d / 2}\right)\right)\right\|^{2} \\
& \quad \leq c \sum_{p=0}^{l-1} \int_{\mathbb{K} \cap\left(\mathcal{V}_{d} \backslash \mathcal{V}_{d / 2}\right)} r^{2\left(\beta^{2}-l+1+p\right)} \theta^{2\left(\gamma^{2}-l+1+p\right)}\left|\nabla_{x}^{p}\left[\mathbf{L}\left(\nabla_{x}\right), \chi_{d}\right]\left(u-\sum_{ \pm} \chi^{ \pm} u^{ \pm}\right)\right|^{2} d x \\
& \quad \leq c \sum_{q=0}^{l+1} \int_{\mathbb{K} \cap\left(\mathcal{V}_{d} \backslash \mathcal{V}_{d / 2}\right)} r^{2\left(\beta^{2}-\gamma^{2}-l+1+p\right)} \theta^{2\left(\gamma^{2}-l+1+p\right)}\left|\nabla_{x}^{q}\left(u-\sum_{ \pm} \chi^{ \pm} u^{ \pm}\right)\right|^{2} d x \\
& \quad \leq c \sum_{q=0}^{l+1} \int_{\mathbb{K} \cap\left(\mathcal{V}_{d} \backslash \mathcal{V}_{d / 2}\right)} r^{2\left(\beta^{1}-l-1+q\right)} \theta^{2(\gamma-l-1+q)}\left|\nabla_{x}^{q}\left(u-\sum_{ \pm} \chi^{ \pm} u^{ \pm}\right)\right|^{2} d x \\
& \quad=c\left\|u-\sum_{ \pm} \chi^{ \pm} u^{ \pm} ;{\stackrel{\mathfrak{V}}{\beta^{1}, \gamma}}^{l+1}\left(\mathbb{K} \cap\left(\mathcal{V}_{d} \backslash \mathcal{V}_{d / 2}\right)\right)\right\|^{2}
\end{aligned}
$$

Here again we have used relations (4.26) and (4.22).

Let us show that for some $\sigma \leq \beta^{1}$ the vector-valued function $\mathfrak{U}$ belongs to the subspace $\mathfrak{\mathfrak { V }}_{\sigma, \gamma^{2}}^{l+1,0}(\mathbb{K})_{\mathfrak{N}}^{k}$. Due to the inequalities $\gamma>\gamma^{2}, \sigma \leq \beta^{1}$, and relations (4.24) on the support of the cutoff $1-\chi_{d}$, the first summand on the right-hand side in (4.21) lies in the spaces

$$
\stackrel{\circ}{\mathfrak{V}}_{\beta^{1}, \gamma}^{l+1}(\mathbb{K})^{k} \subset \mathfrak{\mathfrak { V }}_{\beta^{1}, \gamma}^{l+1,0}(\mathbb{K})^{k} \subset \stackrel{\circ}{\mathfrak{V}}_{\sigma, \gamma^{2}}^{l+1,0}(\mathbb{K})^{k}
$$


The following computations, mainly repeating (4.25), show that the summands $v^{ \pm}$belong to the same spaces (4.30):

$$
\begin{aligned}
& \left\|v^{ \pm} ; \mathfrak{V}_{\beta^{1}, \gamma^{2}}^{l+1,0}(\mathbb{K})\right\|^{2} \\
& \leq c \sum_{m=1}^{\infty} \sum_{p=0}^{l+1}\left\|\nabla_{x}^{p} v^{ \pm} ; C^{0}\left(\xi^{m 0 \pm}\right)\right\|^{2} \int_{\xi^{m 0 \pm}} r^{2\left(\beta^{1}-l-1+p\right)} \theta^{2\left(\gamma^{2}-l-1+p+\delta_{p, 0}\right)} d x \\
& \leq c \sum_{m=1}^{\infty}\left\|\left(1+r^{2}\right)^{\left(\beta^{1}-\gamma\right) / 2} v^{ \pm} ; H^{l+3}\left(\xi^{m 0 \pm}\right)\right\|^{2} \\
& \quad \times \sum_{p=0}^{l+1} r_{m}^{2\left(\beta^{1}-l-1+p\right)} r_{m}^{-2\left(\gamma^{2}-l-1+p+\delta_{p, 0}\right)} r_{m}^{2\left(\gamma-\beta^{1}\right)} \\
& \leq c\left\|\left(1+r^{2}\right)^{\left(\beta^{1}-\gamma\right) / 2} v^{ \pm} ; H^{l+3}(\mathbb{K})\right\|^{2} \leq c\left\|u ; \mathfrak{V}_{\beta^{1}+2, \gamma+2}^{l+3,0}(G)\right\| .
\end{aligned}
$$

Note that the total exponent of the power of $r_{m}$ under the summation sign equals $2\left(\gamma-\gamma^{2}-\delta_{p, 0}\right)$ and turns out to be negative. For the remaining terms in (4.21) we have

$$
\begin{aligned}
&\left\|\chi^{ \pm} u^{ \pm} ; \mathfrak{V}_{\sigma, \gamma^{2}}^{l+1,0}(\mathbb{K})\right\|^{2} \\
& \leq c\left\|\left(1+r^{2}\right)^{\left(\beta^{1}-\gamma-1\right) / 2} u^{ \pm} ; C^{l+1}\left((\partial \mathbb{K})^{ \pm}\right)\right\|^{2} \\
& \times \sum_{p=0}^{l+1} \int_{R}^{\infty} r^{2\left(\sigma-l-1+p-\beta^{1}+\gamma+1\right)} r d r \int_{-\alpha}^{\alpha} \theta^{2\left(\gamma^{2}-l-1+p+\delta_{p, 0}\right)} d \theta .
\end{aligned}
$$

The integrals over the arc $\Upsilon=(-\alpha, \alpha)$ converge due to condition (1.31) for the index $\gamma^{2}$. The integral over the ray $(R,+\infty)$ can be made convergent by the appropriate choice of $\sigma$. Comparing the definition of the norms (1.20) and (1.29), we see that

$$
u \in \mathfrak{V}_{\beta^{1}+2, \gamma+2}^{l+3,0}(G)^{k} \subset \mathfrak{V}_{\beta^{1}+2-1, \gamma+2}^{l+3}(G)^{k} .
$$

Therefore, similarly to the relation (4.19), the norm on the right-hand side of (4.31) does not exceed $c\left\|u \in \mathfrak{V}_{\beta^{1}+2, \gamma+2}^{l+3,0}(G)\right\|$. Finally, the vector-valued function $\mathfrak{U}$ belongs to the class $H_{\text {loc }}^{l+1}(\overline{\mathbb{K}})^{k}$, and hence satisfies the integral identity (2.37) in which the substitutes $u \mapsto \mathfrak{U}$ and $f \mapsto \mathfrak{F}$ are made.

Thus, we have verified that $\mathfrak{U} \in \mathfrak{V}_{\sigma, \gamma^{2}}^{l+1,0}(\mathbb{K})_{\mathfrak{N}}^{k}$ and $\mathfrak{F} \in \mathfrak{V}_{\beta^{2}, \gamma^{2}}^{l-1}(\mathbb{K})^{k}$. Since $\mathfrak{U}$ and $\mathfrak{F}$ vanish inside the circle $\mathbb{B}_{R}$, the assumptions of Theorem 2.2 are satisfied with the role of indices $\beta^{1}, \beta^{2}$, and $\gamma$ played by $\sigma, \beta^{2}$, and $\gamma^{2}$, respectively. Therefore, the vector-valued function $\mathfrak{U}$ admits representation (2.8), i.e.,

$$
\mathfrak{U}(x)=\chi_{\infty}(r) \sum c_{(q)}^{j, p} U_{(q)}^{j, p}(x)+\widetilde{\mathfrak{U}}(x), \quad \widetilde{\mathfrak{U}} \in \mathfrak{V}_{\beta^{2}, \gamma^{2}}^{l+1,0}(\mathbb{K})^{k} .
$$

Here the summation is over the eigenvalues of the pencil (2.4) between the lines $\Lambda_{l-\beta^{2}}$ and $\Lambda_{l-\sigma}$; however, the line $\Lambda_{l-\sigma}$ should be replaced by the line $\Lambda_{l-\beta^{1}}$ because the coefficients $c_{(q)}^{j, p}$ vanish in the case

$$
l-\sigma>\operatorname{Re} \lambda_{q}>l-\beta^{1} .
$$

Indeed, in the representation (4.32) 1 restricted to the region

$$
\mathbb{K}_{R}^{1}=\left\{x: r>R,|\varphi|<\alpha_{1}<\alpha\right\},
$$

the left-hand side coincides with the original solution $u \in V_{\beta^{1}}^{l+1}\left(\mathbb{K}_{R}^{1}\right)^{k}$, and the asymptotic remainder $\widetilde{\mathfrak{U}}$ belongs to the Kondrat'ev space $V_{\beta^{2}}^{l+1}\left(\mathbb{K}_{R}^{1}\right)^{k}$, but the polynomial-logarithmic 
solutions (2.5) corresponding to the eigenvalues $\lambda_{q}$ in formula (4.33) do not lie in either of these spaces. On the other hand, since the eigenfunctions $\Phi_{(q)}^{j, 0}$ of the elliptic problem (2.4) cannot vanish identically on the arc $\left(-\alpha_{1}, \alpha_{1}\right)$ of positive length, the only remaining possibility is $c_{(q)}^{j, p}=0$.

Due to the choice of the cut-off function $\chi_{d}$ and the correcting summands $v^{ \pm}$, the vector-valued function (4.21) coincides with the solution $u$ at least on the set $\omega_{R}^{0}$, i.e., on the sector with parabolic cuts (see formula (1.2)). Following recommendations in 3.2, we include the blocks (3.17) in the asymptotic expansion and rewrite the representation (4.21) as follows:

$$
u(x)=\chi_{\infty}(r) \sum c_{(q)}^{j, p} \mathcal{U}_{(q)}^{j, p}(x)+\widetilde{u}^{1}(x)+\widetilde{u}^{2}(x), \quad x \in \omega_{R}^{0} .
$$

Here the first part of the remainder $\widetilde{u}^{1}=\widetilde{\mathfrak{U}}$ satisfies $(4.32)_{2}$, and the second part

$$
\widetilde{u}^{2}=-\chi_{\infty} \sum c_{(q)}^{j, p} r^{\lambda_{q}-1} \sum_{ \pm} \chi^{ \pm} \mathcal{V}_{(q)}^{j, p \pm}
$$

belongs to the space $\mathfrak{V}_{\mu, \gamma}^{l+1,1}\left(\omega_{R}^{0}\right)^{k}$ for each $\mu \in \mathbb{R}$ since the $\mathcal{V}_{(q)}^{j, p \pm}\left(y^{ \pm}, \log r\right)$ decrease exponentially as $y_{2}^{ \pm} \rightarrow+\infty$.

4.4. The estimate of the remainder near the asymptotic boundary. Our immediate goal is to show that formula (4.34) yields an asymptotic expansion of the solution $u$ on the set $G_{R} \backslash \overline{\omega_{R}^{0}}=G_{R}^{+} \cup G_{R}^{-}$as well, where $G_{R}^{ \pm}=\left\{x \in G_{R}: y_{2}^{ \pm}<a_{ \pm}\left(y_{1}^{ \pm}\right)^{s}\right\}$. The difference

$$
\widetilde{u}(x)=u(x)-\chi_{\infty}(r) \sum c_{(q)}^{j, p} \mathcal{U}_{(q)}^{j, p \pm}(x)
$$

satisfies the following mixed boundary value problem:

$$
\begin{gathered}
L\left(x, \nabla_{x}\right) \widetilde{u}(x)=f^{ \pm}(x), \quad x \in G_{R}^{ \pm} ; \quad N\left(x, \nabla_{x}\right) \widetilde{u}(x)=g^{ \pm}(x), x \in \Gamma_{R}^{ \pm} ; \\
\widetilde{u}(x)=0, x \in \partial G_{R}^{ \pm} \cap \partial \mathbb{B}_{R} ; \quad \widetilde{u}(x)=\widetilde{u}^{1}(x)+\widetilde{u}^{2}(x), x \in \partial G_{R}^{ \pm} \backslash\left(\Gamma_{R}^{ \pm} \cup \partial \mathbb{B}_{R}\right) .
\end{gathered}
$$

In 3.2 we have shown that the new right-hand sides $f^{ \pm}$and $g^{ \pm}$in the problem (4.37) 1 , i.e., the previous $f$ and $g$ perturbed by mismatches of the subtrahend in (4.36), belong to the spaces $\mathfrak{V}_{\beta^{2}, \gamma}^{l-1}(G)^{k}$ and $\mathfrak{V}_{\beta^{2}, \gamma}^{l-1 / 2}(\partial G)^{k}$, respectively. Therefore, to apply the next lemma, it remains to understand the properties of the remainders $\widetilde{u}^{1}$ and $\widetilde{u}^{2}$.

Lemma 4.2. Suppose $l-\gamma>0$ is small and $\widetilde{u} \in \mathfrak{V}_{\beta^{1}, \gamma}^{l+1,0}(G)^{k}$ is a solution of the problem (4.37) with right-hand sides

$$
\begin{gathered}
f^{ \pm} \in \mathfrak{V}_{\beta^{2}, \gamma}^{l-1}(G)^{k}, \quad g^{ \pm} \in \mathfrak{V}_{\beta^{2}, \gamma}^{l-1 / 2}(\partial G)^{k}, \\
\widetilde{u}^{1}+\widetilde{u}^{2}=\widetilde{u}^{0}, x \in \partial G_{R}^{ \pm} \backslash\left(\Gamma_{R}^{ \pm} \cup \partial \mathbb{B}_{R}\right), \quad \widetilde{u}^{0} \in \mathfrak{V}_{\beta^{0}-l, \gamma-l}^{1,0}\left(G_{R}^{ \pm}\right)^{k} .
\end{gathered}
$$

If $s \in\left(0, \beta^{2}-\beta^{0}\right]$, we have $\widetilde{u} \in \mathfrak{V}_{\beta^{0}-l, \gamma-l}^{1,0}\left(G_{R}^{ \pm}\right)^{k}$ and the norm $\left\|\widetilde{u} ; \mathfrak{V}_{\beta^{0}-l, \gamma-l}^{1,0}\left(G_{R}^{ \pm}\right)\right\|$does not exceed a constant multiplied by the sum of the norms of the vector-valued functions (4.38) in these spaces.

Proof. We introduce the weight function

$$
\mathcal{R}(x)= \begin{cases}\left(1+\varepsilon^{2} r^{2}\right)^{\left(\beta^{0}-l\right) / 2}\left(\left(1+r^{2}\right)^{-1}+(\alpha-|\varphi|)^{2}\right)^{(\gamma-l) / 2}, & r \leq t ; \\ \left(1+\varepsilon^{2} t^{2}\right)^{N / 2}\left(1+\varepsilon^{2} r^{2}\right)^{\left(\beta^{0}-l-N\right) / 2}\left(\left(1+r^{2}\right)^{-1}+(\alpha-|\varphi|)^{2}\right)^{(\gamma-l) / 2}, & r \geq t,\end{cases}
$$

with piecewise smooth derivatives, which depends on three parameters: the small parameter $\varepsilon$ and the large parameter $N$ will be chosen later, and the parameter $t>R$ grows unboundedly. Let us emphasize that (4.39) 1 is equivalent to the weight factor at 
the gradient $\nabla_{x} \widetilde{u}$ in the norm $\left\|\widetilde{u} ; \mathfrak{V}_{\beta^{0}-l, \gamma-l}^{1,0}(G)\right\|$ (see definitions (1.29) and (1.21)). The following "key" inequality is satisfied:

$$
\left|\nabla_{x} \mathcal{R}(x)\right| \leq c\left(\varepsilon^{1-s}+|\gamma-l|\right)\left(r^{s}+r|\alpha-| \varphi||\right)^{-1} \mathcal{R}(x), \quad x \in G_{R}^{ \pm} .
$$

Indeed, we have

$$
\begin{aligned}
& \mathcal{R}(x)^{-1}\left|\nabla_{x} \mathcal{R}(x)\right| \\
& \quad \leq c\left\{\frac{\varepsilon^{2} r}{1+\varepsilon^{2} r^{2}}+|\gamma-l|\left(\frac{r}{\left(1+r^{2}\right)^{2}}+\frac{1}{r}|\alpha-| \varphi||\right)\left(\left(1+r^{2}\right)^{-1}+(\alpha-|\varphi|)^{2}\right)^{-1}\right\} .
\end{aligned}
$$

By the definition of the sets $G_{R}^{ \pm}$we have

$$
0 \leq r^{1-s}|\alpha-| \varphi|| \leq C, \quad x \in G_{R}^{ \pm} .
$$

Therefore, the first term in braces in (4.41) does not exceed

$$
c \frac{\varepsilon^{1+s} r^{1+s}}{1+\varepsilon^{2} r^{2}} \varepsilon^{1-s} r^{-s} \leq c \varepsilon^{1-s} r^{-s} \leq c \varepsilon^{1-s}\left(r^{s}+r|\alpha-| \varphi||\right)^{-1} .
$$

The conditions $s \in(0,1)$ and $r \geq R$ make the formula

$$
\frac{r}{\left(1+r^{2}\right)^{2}}\left(\left(1+r^{2}\right)^{-1}+(\alpha-|\varphi|)^{2}\right)^{-1} \leq \frac{r}{1+r^{2}} \leq c r^{-s} \leq c\left(r^{s}+r|\alpha-| \varphi||\right)^{-1}
$$

obvious. Finally, using simple algebraic transformations, we deduce the formula

$$
r^{-1}|\alpha-| \varphi||\left(\left(1+r^{2}\right)^{-1}+(\alpha-|\varphi|)^{2}\right)^{-1} \leq c\left(r^{s}+r|\alpha-| \varphi||\right)^{-1}
$$

again from (4.42). Verification of the inequality (4.40) us completed.

The vector-valued function $\widetilde{u}$ satisfies the following integral identity:

$$
\left(\mathbf{A} \mathcal{D}\left(\nabla_{x}\right) \widetilde{u}, \mathcal{D}\left(\nabla_{x}\right) \phi\right)_{G_{R}^{ \pm}}=\left(f^{ \pm}, \phi\right)_{G_{R}^{ \pm}}+\left(g^{ \pm}, \phi\right)_{\Gamma_{R}^{ \pm}}+\left(\mathbf{A} \mathcal{D}\left(\nabla_{x}\right) \widetilde{u}^{0}, \mathcal{D}\left(\nabla_{x}\right) \phi\right)_{G_{R}^{ \pm}},
$$

where $\phi \in C_{c}^{\infty}\left(G_{R}^{ \pm} \cup \Gamma_{R}^{ \pm}\right)^{k}$ is a smooth test function with compact support vanishing near the set where the Dirichlet conditions (4.37) 2 are imposed. Now we substitute the product $\mathcal{R} V$, where $V=\mathcal{R}\left(\widetilde{u}-\widetilde{u}^{0}\right)$, in (4.43) instead of $\phi$. If the number $N$ in definition (4.39) is chosen to be sufficiently large, then all integrals in (4.43) converge by the hypotheses of the lemma, which justifies the above substitution: one must approximate $\widetilde{u}$ in the norm of the space $\mathfrak{V}_{\beta^{0}-l, \gamma-l}^{1,0}(G)$ and pass to the limit. We present simple transformations, which were already used in (2.27):

$$
\begin{aligned}
(\mathbf{A} \mathcal{D}( & \left.\left.\nabla_{x}\right)\left(\widetilde{u}-\widetilde{u}^{0}\right), \mathcal{D}\left(\nabla_{x}\right) \mathcal{R} V\right)_{G_{R}^{ \pm}} \\
= & \left(\mathbf{A} \mathcal{R} \mathcal{D}\left(\nabla_{x}\right)\left(\widetilde{u}-\widetilde{u}^{0}\right), \mathcal{D}\left(\nabla_{x}\right) V\right)_{G_{R}^{ \pm}} \\
& +\left(\mathbf{A} \mathcal{R} \mathcal{D}\left(\nabla_{x}\right)\left(\widetilde{u}-\widetilde{u}^{0}\right), \mathcal{R}^{-1}\left[\mathcal{D}\left(\nabla_{x}\right), \mathcal{R}\right] V\right)_{G_{R}^{ \pm}} \\
= & \left(\mathbf{A} \mathcal{D}\left(\nabla_{x}\right) V, \mathcal{D}\left(\nabla_{x}\right) V\right)_{G_{R}^{ \pm}} \\
& -\left(\mathbf{A} \mathcal{R}^{-1}\left[\mathcal{D}\left(\nabla_{x}\right), \mathcal{R}\right] V, \mathcal{D}\left(\nabla_{x}\right) V\right)_{G_{R}^{ \pm}}+\left(\mathbf{A} \mathcal{D}\left(\nabla_{x}\right) V, \mathcal{R}^{-1}\left[\mathcal{D}\left(\nabla_{x}\right), \mathcal{R}\right] V\right)_{G_{R}^{ \pm}} \\
& -\left(\mathbf{A} \mathcal{R}^{-1}\left[\mathcal{D}\left(\nabla_{x}\right), \mathcal{R}\right] V, \mathcal{R}^{-1}\left[\mathcal{D}\left(\nabla_{x}\right), \mathcal{R}\right] V\right)_{G_{R}^{ \pm}}
\end{aligned}
$$

Here $\left[\mathcal{D}\left(\nabla_{x}\right), \mathcal{R}\right]=\mathcal{D}\left(\nabla_{x}\right) \mathcal{R}$ is a matrix-valued function for which the desired estimate of its module follows from inequality (4.40).

Relations (4.44) and (4.40) together with the weight Korn inequality

$$
\left\|\nabla_{x} V ; L_{2}\left(G_{R}^{ \pm}\right)\right\|^{2}+\left\|\left(r^{s}+r|\alpha-| \varphi||\right)^{-1} V ; L_{2}\left(G_{R}^{ \pm}\right)\right\|^{2} \leq c\left\|\mathcal{D}\left(\nabla_{x}\right) V ; L_{2}\left(G_{R}^{ \pm}\right)\right\|^{2}
$$


(to be verified below) show that for small (fixed) $\varepsilon$ and $l-\gamma$ we have the estimate

$$
\begin{array}{r}
\left(\mathbf{A} \mathcal{D}\left(\nabla_{x}\right) V, \mathcal{D}\left(\nabla_{x}\right) V\right)_{G_{R}^{ \pm}} \leq c\left(\left|\left(\mathcal{R} f^{ \pm}, V\right)_{G_{R}^{ \pm}}\right|+\left|\left(\mathcal{R} g^{ \pm}, V\right)_{\Gamma_{R}^{ \pm}}\right|\right) \\
\leq c\left\|\mathcal{D}\left(\nabla_{x}\right) V ; L_{2}\left(\Gamma_{R}^{ \pm}\right)\right\|\left\{\left\|\left(r^{s}+r|\alpha-| \varphi||\right) \mathcal{R} f^{ \pm} ; L_{2}\left(\Gamma_{R}^{ \pm}\right)\right\|\right. \\
\left.+\left\|r^{s / 2} \mathcal{R} g^{ \pm} ; L_{2}\left(\Gamma_{R}^{ \pm}\right)\right\|\right\} .
\end{array}
$$

Here we have used the trace inequality

$$
\left\|r^{-s / 2} V ; L_{2}\left(\Gamma_{R}^{ \pm}\right)\right\| \leq c\left(\left\|\mathcal{D}\left(\nabla_{x}\right) V ; L_{2}\left(G_{R}^{ \pm}\right)\right\|+\left\|\left(r^{s}+r|\alpha-| \varphi||\right)^{-1} V ; L_{2}\left(G_{R}^{ \pm}\right)\right\|\right),
$$

which can be easily proved using the classical scheme 41]. The expression in braces on the right-hand side of (4.46) does not exceed the sum of norms of vector-valued functions (4.38) 1 in the above spaces. This follows from the relations ensured by formulas (4.38), (4.42), (1.20), (1.21), Lemma 1.1, 1), and the condition $s \leq \beta^{2}-\beta^{0}$ :

$$
\begin{gathered}
\left(r^{s}+r|\alpha-| \varphi||\right) \mathcal{R}(x) \leq c r^{\beta^{2}-\gamma}(1+r|\alpha-| \varphi||), \quad x \in G_{R}^{ \pm}, \\
r^{s / 2} \mathcal{R}(x) \leq c r^{\beta^{2}-\gamma}, \quad x \in \Gamma_{R}^{ \pm} .
\end{gathered}
$$

Therefore, as $t \rightarrow+\infty$, the right-hand side of (4.46) is bounded, and we can pass to the limit on the left-hand side. In the process, the weight factor $\mathcal{R}(x)$ becomes

$$
\left(1+\varepsilon^{2} r^{2}\right)^{\left(\beta^{0}-l\right) / 2}\left(\left(1+r^{2}\right)^{-1}+(\alpha-|\varphi|)^{2}\right)^{(\gamma-l) / 2}
$$

everywhere on the set $G_{R}^{ \pm}$. As a result, we establish the finiteness of the norms and the relation

$$
\begin{aligned}
& \left\|r^{\beta^{0}-\gamma}\left(r^{-1}+|\alpha-| \varphi||\right)^{\gamma-l} \nabla_{x}\left(\widetilde{u}-\widetilde{u}^{0}\right) ; L_{2}\left(\omega_{R}^{ \pm}\right)\right\|^{2} \\
& \quad+\left\|r^{\beta^{0}-\gamma}\left(r^{-1}+|\alpha-| \varphi||\right)^{\gamma-l}\left(r^{s}+r|\alpha-| \varphi||\right)^{-1}\left(\widetilde{u}-\widetilde{u}^{0}\right) ; L_{2}\left(\omega_{R}^{ \pm}\right)\right\|^{2} \\
& \quad \leq c\left(\left\|f^{ \pm} ; \mathfrak{V}_{\beta^{2}, \gamma}^{l-1}(G)\right\|+\left\|g^{ \pm} ; \mathfrak{V}_{\beta^{2}, \gamma}^{l-1 / 2}(\partial G)\right\|^{2}\right) .
\end{aligned}
$$

On the left-hand side of (4.47), the factor at $\left|\nabla_{x}\left(\widetilde{u}-\widetilde{u}^{0}\right)\right|^{2}$ is equivalent to a similar factor in the norm $\left\|\widetilde{u}-\widetilde{u}^{0} ; \mathfrak{V}_{\beta^{0}-l, \gamma-l}^{1,0}\left(G_{R}^{ \pm}\right)\right\|$(see definition (1.29)) and the factor at $\left|\widetilde{u}-\widetilde{u}^{0}\right|^{2}$ is greater than the corresponding factor in the norm (due to relation (4.41)). In other words, the required inclusions and the estimate are established for the difference $\widetilde{u}-\widetilde{u}^{0}$, hence for the solution $\widetilde{u}$.

It remains to establish the weight Korn inequality (4.45).

From the parabolic region $\omega_{R}^{ \pm}$(see (1.2)) we cut off the sets

$$
\omega_{R}^{j \pm}=\left\{x \in \omega_{R}^{ \pm}: R j^{1 /(1-s)}<y_{1}^{ \pm}<R j^{1 /(1-s)}+\frac{R}{1-s} j^{s /(1-s)}\right\}, \quad j \in \mathbb{N} .
$$

Due to the simple relations

$$
\left(\frac{j+1}{j}\right)^{1 /(1-s)}<1+\frac{1}{1-s} j^{-1}, \quad \frac{s}{1-s}-\frac{1}{1-s}=-1,
$$

the sets $\omega_{R}^{j \pm}$ and $\omega_{R}^{j+1 \pm}$ have nonempty intersections, i.e., $\left\{\omega_{R}^{j \pm}\right\}_{j \in \mathbb{N}}$ is a covering of the support of the vector-valued function $V=\widetilde{u}-\widetilde{u}^{0}$ (we recall that all functions vanish inside the circle of radius $3 R / 2$ ). The change of coordinates

$$
x \mapsto \eta^{j \pm}=j^{-s /(1-s)}\left(y_{1}^{ \pm}-R j^{1 /(1-s)}, y_{2}^{ \pm}\right)
$$


transforms $\omega_{R}^{j \pm}$ into the set

$$
\widetilde{\omega}_{R}^{j \pm}=\left\{\eta^{j \pm}: 0<\eta_{1}^{j \pm}<\frac{R}{1-s}, 0<\eta_{2}^{j \pm}<a_{ \pm}\left(R+j^{-1} \eta_{1}^{ \pm}\right)^{s}\right\},
$$

which is of unit size and is contained in the standard set

$$
\omega_{R}^{0 \pm}=\left\{\eta^{j \pm}: 0<\eta_{1}^{j \pm}<\frac{R}{1-s}, 0<\eta_{2}^{j \pm}<a_{ \pm}\left(R+\eta_{1}^{ \pm}\right)^{s}\right\} .
$$

Let us extend the vector-valued function $V$ by zero across the curve $\partial G_{R}^{ \pm} \backslash\left(\Gamma_{R}^{ \pm} \cup \partial \mathbb{B}_{R}\right)$ and apply to this extension,

$$
V^{j \pm}\left(\eta^{j \pm}\right)=V\left(j^{s /(1-s)} \eta_{1}^{ \pm}+R j^{1 /(1-s)}, j^{s /(1-s)} \eta_{2}^{ \pm}\right),
$$

the Korn inequality established in [1, which holds since $V^{j \pm}$ vanishes on a part of the boundary:

$$
\left\|\nabla_{\eta^{j \pm}} V^{j \pm} ; L_{2}\left(\omega_{R}^{0 \pm}\right)\right\|^{2}+\left\|V^{j \pm} ; L_{2}\left(\omega_{R}^{0 \pm}\right)\right\|^{2} \leq c\left\|\mathcal{D}^{ \pm}\left(\nabla_{\eta^{j \pm}}\right) V^{j \pm} ; L_{2}\left(\omega_{R}^{0 \pm}\right)\right\|^{2} .
$$

Take into account the integrated over $\eta_{1}^{j \pm} \in(0, R /(1-s))$ Hardy inequality

$$
\left\|\left(2 a_{ \pm}\left(R+j^{-1} \eta_{1}^{j \pm}\right)^{s}-\eta_{2}^{j \pm}\right)^{-1} V^{j \pm} ; L_{2}\left(\omega_{R}^{0 \pm}\right)\right\|^{2} \leq 4\left\|\frac{\partial V^{j \pm}}{\partial \eta_{2}^{j \pm}} ; L_{2}\left(\omega_{R}^{0 \pm}\right)\right\|^{2}
$$

and return to coordinates $x$. As a result, we obtain the estimate

$$
\left\|\nabla_{x} V ; L_{2}\left(\omega_{R}^{j \pm}\right)\right\|^{2}+\left\|\left(2 a_{ \pm}\left(y_{1}^{ \pm}\right)^{s}-y_{2}^{ \pm}\right)^{-1} V ; L_{2}\left(\omega_{R}^{j \pm}\right)\right\|^{2} \leq c\left\|\mathcal{D}\left(\nabla_{x}\right) V ; L_{2}\left(\omega_{R}^{j \pm}\right)\right\|^{2} .
$$

Since for a large radius $R$ all points $x \in \omega_{R}^{ \pm}$satisfy the relation

$$
2 a_{ \pm}\left(y_{1}^{ \pm}\right)^{s}-y_{2}^{ \pm} \geq a_{ \pm}\left(y_{1}^{ \pm}\right)^{s} \geq c\left(r^{s}+r(\alpha-|\varphi|)\right),
$$

summing up inequalities (4.52) for $j \in \mathbb{N}$ leads to formula (4.45) with the region $G_{R}^{ \pm}$ replaced by the region $\omega_{R}^{ \pm}$. To extend the Korn inequality to the set $G_{R}^{ \pm} \backslash \omega_{R}^{ \pm}$, we cover this set by trapezoids with curved bases

$$
\xi^{m \pm}=\left\{x: y_{1}^{ \pm}-R-m \in(-1,0), H^{0}>y_{2}^{ \pm}>H^{ \pm}\left(y_{1}^{ \pm}\right)\right\}, \quad m \in \mathbb{N}
$$

(cf. constructions $(4.5)_{1}$ ) and denote by $\xi^{m 0 \pm}$ the rectangles $\xi^{m \pm} \cap \mathbb{K}_{R}$. Let $\mathbf{p}^{1}, \ldots, \mathbf{p}^{Q}$ be the basis in the algebraic subspace $\mathbf{P}$ of vector-valued polynomials (columns) on which the sesquilinear form (1.6) degenerates. Here we denote $\mathbf{p}^{n}=\left(\delta_{1, n}, \ldots, \delta_{k, n}\right)^{\top}$ and $\left(\mathbf{p}^{m}, \mathbf{p}^{n}\right)_{\xi^{m 0 \pm}}=0$ for $n=1, \ldots, k, m=k+1, \ldots, Q$. in the set $\xi^{m \pm}$ we represent the vector-valued function $V$ as follows:

$$
V(x)=V^{\perp}(x)+\sum_{q=1}^{Q} c_{q} \mathbf{p}^{q}(x)=V^{\perp}(x)+\mathbf{p}(x) a
$$

and impose on the component $V^{\perp}$ the orthogonality condition

$$
\int_{\xi^{m 0 \pm}} \mathbf{p}(x)^{\top} V^{\perp}(x) d x \in \mathbb{C}^{Q} .
$$

Here $\mathbf{p}=\left(\mathbf{p}^{1}, \ldots, \mathbf{p}^{Q}\right)$ as a $k \times Q$ matrix, $a=\left(a_{1}, \ldots, a_{Q}\right)^{\top}$ is the column of the coefficients, and $a^{\prime}=\left(a_{1}, \ldots, a_{k}\right)^{\top}, a^{\prime \prime}=\left(a_{k+1}, \ldots, a_{Q}\right)^{\top}$. The Gram matrix

$$
\mathfrak{P}=\int_{\xi^{m 0 \pm}} \mathbf{p}(x)^{\top} \mathbf{p}(x) d x
$$


is of size $Q \times Q$ and is also symmetric, positive definite, and block-diagonal. According to (4.55) we have

$$
\mathfrak{P} a=\int_{\xi^{m 0 \pm}} \mathbf{p}(x)^{\top} V(x) d x, \quad\left\|a^{\prime} ; \mathbb{C}^{k}\right\| \leq c\left\|V ; L_{2}\left(\xi^{m 0 \pm}\right)\right\| .
$$

Moreover,

$$
\nabla_{x} V-\nabla_{x} V^{\perp}=\nabla_{x} \mathbf{p}^{1+k}+\cdots+\nabla_{x} \mathbf{p}^{Q} .
$$

The matrix-valued functions $\nabla_{x} \mathbf{p}^{1+k}, \ldots, \nabla_{x} \mathbf{p}^{Q}$ are linearly independent in the space $L_{2}\left(\xi^{m 0 \pm}\right)^{2 \times k}$; hence

$$
\left\|a^{\prime \prime} ; \mathbb{C}^{Q-k}\right\| \leq c\left(\left\|\nabla_{x} V ; L_{2}\left(\xi^{m 0 \pm}\right)\right\|+\left\|\nabla_{x} V^{\perp} ; L_{2}\left(\xi^{m 0 \pm}\right)\right\|\right) .
$$

Since $\mathcal{D}\left(\nabla_{x}\right) \mathbf{p}^{q}=0$, the versions of the Korn inequality established in the book [1],

$$
\begin{aligned}
\left\|V^{\perp} ; H^{1}\left(\xi^{m 0 \pm}\right)\right\|^{2} & \leq c\left\|\mathcal{D}\left(\nabla_{x}\right) V^{\perp} ; L_{2}\left(\xi^{m 0 \pm}\right)\right\|^{2}=c\left\|\mathcal{D}\left(\nabla_{x}\right) V ; L_{2}\left(\xi^{m 0 \pm}\right)\right\|^{2}, \\
\left\|V^{\perp} ; H^{1}\left(\xi^{m \pm}\right)\right\|^{2} & \leq c\left(\left\|\mathcal{D}\left(\nabla_{x}\right) V^{\perp} ; L_{2}\left(\xi^{m \pm}\right)\right\|^{2}+\left\|V^{\perp} ; L_{2}\left(\xi^{m 0 \pm}\right)\right\|^{2}\right) \\
& \leq c\left\|\mathcal{D}\left(\nabla_{x}\right) V ; L_{2}\left(\xi^{m \pm}\right)\right\|^{2},
\end{aligned}
$$

together with estimates (4.56) and (4.57) for the columns $c^{\prime}$ and $c^{\prime \prime}$, lead to the relation

$$
\begin{aligned}
& \left\|\nabla_{x} V ; H^{1}\left(\xi^{m \pm}\right)\right\|^{2}+r_{m}^{-2 s}\left\|V ; H^{1}\left(\xi^{m \pm}\right)\right\|^{2} \\
& \quad \leq c\left(\left\|V^{\perp} ; H^{1}\left(\xi^{m \pm}\right)\right\|^{2}+r_{m}^{-2 s}\left\|a^{\prime} ; \mathbb{C}^{k}\right\|^{2}+\left\|a^{\prime \prime} ; \mathbb{C}^{Q-k}\right\|^{2}\right) \\
& \quad \leq c\left(\left\|\mathcal{D}\left(\nabla_{x}\right) V ; L_{2}\left(\xi^{m \pm}\right)\right\|^{2}+\left\|\nabla_{x} V ; L_{2}\left(\xi^{m 0 \pm}\right)\right\|^{2}+r_{m}^{-2 s}\left\|V ; L_{2}\left(\xi^{m 0 \pm}\right)\right\|^{2}\right),
\end{aligned}
$$

where $r_{m}$ is the center of mass of the figure $\xi^{m 0 \pm}$. As usual, we move the weight factors under the integral sign and sum up the modified inequalities (4.58) for $m \in \mathbb{N}$. As a result, we obtain the required formula

$$
\begin{aligned}
& \left\|\nabla_{x} V ; L_{2}\left(G_{R}^{ \pm} \backslash \omega_{R}^{ \pm}\right)\right\|^{2}+\left\|\left(r^{s}+r|\alpha-| \varphi||\right)^{-1} V ; L_{2}\left(G_{R}^{ \pm} \backslash \omega_{R}^{ \pm}\right)\right\|^{2} \\
& \quad \leq c\left(\left\|\mathcal{D}\left(\nabla_{x}\right) V ; L_{2}\left(G_{R}^{ \pm}\right)\right\|^{2}+\left\|\nabla_{x} V ; L_{2}\left(\omega_{R}^{ \pm}\right)\right\|^{2}+\left\|\left(r^{s}+r|\alpha-| \varphi||\right)^{-1} V ; L_{2}\left(\omega_{R}^{ \pm}\right)\right\|^{2}\right) .
\end{aligned}
$$

The proof of Lemma 4.2 is complete.

Now we must prove that the remainders $\widetilde{u}^{1}$ and $\widetilde{u}^{2}$ in representation (4.34) on the set $\omega_{R}^{0}$ admit extensions to the parabolic regions $\omega_{R}^{ \pm}$in the class $\mathfrak{V}_{\beta^{0}-l, \gamma-l}^{1,0}$. For an extension of the vector-valued function (4.35) we take the product $\chi_{\omega}^{ \pm} \widetilde{u}^{2}$, where $\chi_{\omega}^{ \pm}(x)=$ $1-\chi_{0}\left(r^{1-s}(\alpha-|\varphi|)\right)$; here $\chi_{0} \in C^{\infty}(\mathbb{R}), \chi_{0}(t)=1$ for $|t|<a / 3$, and $\chi_{0}(t)=0$ for $|t|>2 a / 3, a=\min \left\{a_{+}, a_{-}\right\}$. Since all components $\mathcal{V}_{(q)}^{j, p \pm}$ exponentially decrease, the product belongs to the space $\mathfrak{V}_{\sigma, \gamma}^{l+1,0}\left(\omega_{R}^{ \pm}\right)^{k}$ for all indices $\sigma$ and $\gamma$. The situation with the summand $\widetilde{u}^{1} \in \mathfrak{V}_{\beta^{2}, \gamma^{2}}^{\circ 1+1,0}(\mathbb{K})^{k}$ is more complicated. By Lemma 2.4 it admits representation (2.54) with $\widetilde{u}^{10} \in \mathfrak{\mathfrak { V }}_{\beta^{2}, \gamma^{2}}(\mathbb{K})^{k}$ and $\widetilde{K}^{ \pm}$; the vector-valued function $t \mapsto \widetilde{K}^{ \pm}(t)$ belongs to the Sobolev-Slobodetskii space $H^{\kappa^{2}}(\mathbb{R})^{k}$ with the exponent

$$
\kappa^{2}=l-\gamma^{2}+\frac{1}{2}=l-\gamma+\beta^{1}-\beta^{2}+\frac{1}{2}
$$

and

$$
\left\|\widetilde{u}^{10} ; \mathfrak{V}_{\beta^{2}, \gamma^{2}}^{l+1}(\mathbb{K})\right\|+\left\|\widetilde{K}^{ \pm} ; H^{\kappa^{2}}(\mathbb{R})\right\| \leq c\left\|\widetilde{u}^{1} ; \mathfrak{\mathfrak { V }}_{\beta^{2}, \gamma^{2}}^{l+1,0}(\mathbb{K})\right\| .
$$

Since $\mathfrak{C}^{ \pm}$are nonlocal operators, we multiply the representation (2.54) by the cut-off function $\chi_{\infty}$ which differs from 1 only at the points where $\widetilde{u}^{1}=0$. 
As before, for the extension of $\widetilde{u}^{10}$ we take $\chi_{\omega}^{ \pm} \widetilde{u}^{10}$. Since

$$
0<c \leq r^{1-s}(\alpha-|\varphi|) \leq C
$$

on the set $\Xi_{R}^{ \pm}=\operatorname{supp} \chi_{\omega}^{ \pm} \cap \omega_{R}^{ \pm}$, formulas (1.29), (1.20), (2.45) 2 , and (4.23) imply

$$
\begin{aligned}
& \left\|\chi_{\infty} \chi_{\omega}^{ \pm} \widetilde{u}^{10} ; \mathfrak{V}_{\beta^{0}, \gamma}^{l+1,0}\left(G_{R}^{ \pm}\right)\right\|^{2} \leq\left\|\chi_{\infty} \chi_{\omega}^{ \pm} \widetilde{u}^{10} ; \mathfrak{V}_{\beta^{0}, \gamma}^{l+1}\left(G_{R}^{ \pm}\right)\right\|^{2} \\
& \leq c \sum_{p=0}^{l+1} \int_{\Xi_{R}^{ \pm}}(1+r)^{2\left(\beta^{0}-\gamma\right)} \rho^{2(\gamma-l-1+p)}\left|\nabla_{x}^{p}\left(\chi_{\infty} \widetilde{u}^{10}\right)\right|^{2} d x \\
& \leq c \sum_{p=0}^{l+1} \int_{\Xi_{\omega}^{ \pm}} r^{2\left(\beta^{0}-l-1\right)}\left(r^{-1}+(\alpha-|\varphi|)\right)^{2(\gamma-l-1+p)}\left|\nabla_{x}^{p}\left(\chi_{\infty} \widetilde{u}^{10}\right)\right|^{2} d x \\
& \leq c \sup _{\Xi_{R}^{ \pm}}\left|r^{2\left(\beta^{0}-\beta^{2}\right)}(\alpha-|\varphi|)^{2\left(\gamma-\gamma^{2}\right)}\right| \sum_{p=0}^{l+1} \int_{\Xi_{R}^{ \pm}} r^{2\left(\beta^{2}-l-1\right)} \theta^{2\left(\gamma^{2}-l-1+p\right)}\left|\nabla_{x}^{p} \widetilde{u}^{10}\right|^{2} d x \\
& \leq c \sup _{\Xi_{R}^{ \pm}}\left|r^{2\left(\beta^{0}-\beta^{2}+(s-1)\left(\beta^{1}-\beta^{2}\right)\right)}\right|\left\|\widetilde{u}^{10} ; \mathfrak{V}_{\beta^{2}, \gamma^{2}}^{l+1}(\mathbb{K})\right\|^{2} \leq c\left\|\widetilde{u}^{10} ; \mathfrak{V}_{\beta^{2}, \gamma^{2}}^{l+1}(\mathbb{K})\right\|^{2} .
\end{aligned}
$$

The last inequality assumes that the supremum is finite, which is the case when, e.g.,

$$
\beta^{0}<\beta^{1}+s\left(\beta^{2}-\beta^{1}\right) .
$$

Remark 4.2. The requirement (4.63) and condition $\beta^{0} \leq \beta^{2}-s$ presented in Lemma 4.2 and used in transformations (4.46)-(4.47) impose some restrictions on the choice of the exponent $s$ in the decomposition of the sector $\mathbb{K}_{R}$ into the regions (1.2). We can take

$$
s=\frac{2\left(\beta^{2}-\beta^{1}\right)}{2+\beta^{2}-\beta^{1}} \in(0,1), \quad \beta^{0}=\beta^{2}-\frac{2\left(\beta^{2}-\beta^{1}\right)}{2+\beta^{2}-\beta^{1}} .
$$

We emphasize that $\beta^{0} \rightarrow \beta^{2}-0$ for $\beta^{1} \rightarrow \beta^{2}-0$. In is clear that the value (4.64) will not work if the line $\Lambda_{l-\beta^{0}}$ contains an eigenvalue of the pencil (2.4). In this case the index $\beta^{0}$ can be slightly increased by moving the line away from the eigenvalue but keeping the inequality (4.63).

The terms $\chi_{\infty} \chi^{ \pm} \mathfrak{C}^{ \pm}\left(\widetilde{K}^{ \pm}\right)$have singularities on the sides of the sector (cf. comments to formula (2.40) $)$. At the same time, they cannot be multiplied by a cut-off function since such a product would not lie in the weight space with the step norm (1.29). We use a new trick. Namely, we smoothen these terms replacing them with the expressions

$$
\begin{aligned}
& \chi_{\infty}(r) \chi^{ \pm}(\varphi) r^{l-\beta^{2} \widetilde{\mathfrak{C}}^{ \pm}}\left(K^{ \pm} ; \varphi, \log r\right), \\
& \widetilde{\mathfrak{C}}^{ \pm}\left(K^{ \pm} ; \varphi, \log r\right)=\int_{\mathbb{R}} X(z) K^{ \pm}\left(\log r-\left(\left(1+r^{2}\right)^{(s-1) / 2}+|\alpha \mp \varphi|\right) z\right) d z .
\end{aligned}
$$

In the next lemma we establish that

$$
\begin{aligned}
\chi_{\infty} \chi^{ \pm} r^{l-\beta^{2}} \widetilde{\mathfrak{C}}^{ \pm}\left(\widetilde{K}^{ \pm}\right) & \in \mathfrak{V}_{\beta^{0}-l, \gamma-l}^{1,0}\left(G_{R}^{ \pm}\right)^{k}, \\
\chi_{\infty} \chi^{ \pm} r^{l-\beta^{2}}\left(\mathfrak{C}^{ \pm}\left(\widetilde{K}^{ \pm}\right)-\widetilde{\mathfrak{C}}^{ \pm}\left(\widetilde{K}^{ \pm}\right)\right) & \in \mathfrak{V}_{\beta^{0}-l, \gamma-l}^{1}\left(\Xi_{R}^{ \pm}\right)^{k} .
\end{aligned}
$$

Therefore, the first expression belongs to the class prescribed by $(4.38)_{2}$, whereas the second can be handled similarly to the remainder $\widetilde{u}^{10}$ : computations (4.62) become only simpler in the case $l=0$. This concludes the verification of $(4.38)_{2}$ and of all the hypotheses of Lemma 4.2 .

Lemma 4.3. Formulas (4.65) hold and the norms of vector-valued functions in these formulas do not exceed $c\left\|\widetilde{K}^{ \pm} ; H^{\kappa^{2}}(\mathbb{R})\right\|$ (see formulas (4.59) and (4.60)). 
Proof. According to relations (2.53) and (2.51) based on the theorem concerning the Fourier transform of a convolution, we have

$$
\mathfrak{C}^{ \pm}\left(\widetilde{K}^{ \pm} ; \varphi, t\right)=\int_{\mathbb{R}} X(z) \widetilde{K}^{ \pm}(t-(\alpha \mp \varphi) z) d z=\int_{\mathbb{R}} \chi(|\eta|(\alpha \mp \varphi)) \widetilde{k}^{ \pm}(\eta) d \eta,
$$

where $\widetilde{k}^{ \pm}$is the Fourier preimage of the vector-valued function $\widetilde{K}^{ \pm}$. Similarly,

$$
\begin{aligned}
\widetilde{\mathfrak{C}}^{ \pm}\left(\widetilde{K}^{ \pm} ; \varphi, t\right) & =\int_{\mathbb{R}} X(z) \widetilde{K}^{ \pm}\left(t-\left(\left(1+r^{2}\right)^{(s-1) / 2}+|\alpha \mp \varphi|\right) z\right) d z \\
& =\int_{\mathbb{R}} \chi\left(|\eta|\left(\left(1+r^{2}\right)^{(s-1) / 2}+|\alpha \mp \varphi|\right)\right) \widetilde{k}^{ \pm}(\eta) d \eta .
\end{aligned}
$$

In view of the second representation,

$$
\begin{aligned}
& \left\|\chi_{\infty} \chi^{ \pm} r^{l-\beta^{2}} \widetilde{\mathfrak{C}}^{ \pm}\left(\widetilde{K}^{ \pm}\right) ; \mathfrak{V}_{\beta^{0}-l, \gamma-l}^{1,0}\left(G_{R}^{ \pm}\right)\right\|^{2} \\
& \leq c \int_{G_{R}^{ \pm}}(1+r)^{2\left(\beta^{0}-\gamma-1+l-\beta^{2}\right)} \rho^{2(\gamma-l)}\left(\left|\widetilde{\mathfrak{C}}^{ \pm}\left(\widetilde{K}^{ \pm}\right)\right|^{2}+(1+r)^{2}\left|\nabla_{x} \widetilde{\mathfrak{C}}^{ \pm}\left(\widetilde{K}^{ \pm}\right)\right|^{2}\right) d x \\
& \leq c \int_{G_{\omega}^{ \pm}}(1+r)^{2\left(\beta^{0}-\beta^{2}-1\right)}\left(r^{-1}+(\alpha-|\varphi|)\right)^{2(\gamma-l)} \\
& \times\left.\left.\left|\int_{\mathbb{R}}\right| \widetilde{k}^{ \pm}(\eta)\right|^{2}\left(|\chi(\cdots)|^{2}+|\eta|^{2}\left|\chi^{\prime}(\cdots)\right|^{2}\right) d \eta\right|^{2} d x \\
& \leq c \int_{G_{\omega}^{ \pm}}(1+r)^{2\left(\beta^{0}-\beta^{2}-1\right)}\left(r^{-1}+(\alpha-|\varphi|)\right)^{2(\gamma-l)} \\
& \times\left(\left\|\widetilde{k}^{ \pm} ; L_{2}(\mathbb{R})\right\|^{2}+\left(r^{s-1}+(\alpha-|\varphi|)\right)^{-2\left(1-\kappa^{2}\right)}\left\|\eta^{\kappa} \widetilde{k}^{ \pm} ; L_{2}(\mathbb{R})\right\|^{2}\right) d x \\
& \leq c\left\|\widetilde{K}^{ \pm} ; H^{\kappa^{2}}(\mathbb{R})\right\|^{2} \int_{R}^{\infty} r^{2\left(\beta^{0}-\beta^{2}-1\right)} \int_{-c r^{-1}}^{C r^{s-1}}\left(r^{-1}+\theta\right)^{2(\gamma-l)} \\
& \times\left(1+\left(r^{s-1}+\theta\right)^{-2\left(1-\kappa^{2}\right)}\right) d \varphi r d r \\
& \leq c\left\|\widetilde{K}^{ \pm} ; H^{\kappa^{2}}(\mathbb{R})\right\|^{2} .
\end{aligned}
$$

Here $\chi^{\prime}$ is an arbitrary cutoff of the function $\chi$ and the dot replaces the same argument of this function as in formula (4.67). The integral with respect to the variable $\theta=\alpha \mp \varphi$ does not exceed

$$
c r^{-2(1-s)\left(\gamma-l-1-\kappa^{2}+1 / 2\right)}=c r^{-2(1-s)\left(\beta^{1}-\beta^{2}\right)} ;
$$

therefore, the double integral converges due to condition (4.63). The estimate (4.69) of this integral with respect to $\theta$ is computed using the change of variable

$$
\begin{aligned}
\int_{-\tau}^{t}(\tau+\theta)^{2(\gamma-l)}(t+ & \theta)^{-2\left(1-\kappa^{2}\right)} d \theta \\
& =t^{2(\gamma-l)-2\left(1-\kappa^{2}\right)+1} \int_{-t^{-1} \tau}^{1}\left(t^{-1} \tau+\psi\right)^{2(\gamma-l)}(1+\psi)^{-2\left(1-\kappa^{2}\right)} d \psi ;
\end{aligned}
$$

here $1 \geq t>\tau$, and taking into account inequality $2(\gamma-l)>-1$, we estimate the latter integral by a constant independent of $\tau / t$. 
Now consider the difference of (4.66) and (4.67) on the set $\Xi_{R}^{ \pm}$. Repeating, to some extent, computations (4.68) and taking into account (4.61), we find

$$
\begin{aligned}
& \| \chi_{\infty} \chi^{ \pm} r^{l-\beta^{2}}\left(\mathfrak{C}^{ \pm}\left(\widetilde{K}^{ \pm}\right)-\widetilde{\mathfrak{C}}^{ \pm}\left(\widetilde{K}^{ \pm}\right)\right) ; \mathfrak{V}_{\beta^{0}-l, \gamma-l}^{1}\left(G_{R}^{ \pm}\right) \|^{2} \\
& \leq c \int_{\Xi_{R}^{ \pm}} r^{2\left(\beta^{0}-l-1+l-\beta^{2}\right)}\left(r^{-1}+\alpha \mp \varphi\right)^{2(\gamma-l-1)} \\
& \times\left(\left|\mathfrak{C}^{ \pm}\left(\widetilde{K}^{ \pm}\right)-\widetilde{\mathfrak{C}}^{ \pm}\left(\widetilde{K}^{ \pm}\right)\right|^{2}+r^{2}\left(r^{-1}+\alpha \mp \varphi\right)^{2}\left|\nabla_{x}\left(\mathfrak{C}^{ \pm}\left(\widetilde{K}^{ \pm}\right)-\widetilde{\mathfrak{C}}^{ \pm}\left(\widetilde{K}^{ \pm}\right)\right)\right|^{2}\right) d x \\
& \leq c \int_{\Xi_{\omega}^{ \pm}} r^{2\left(\beta^{0}-\beta^{2}-1\right)} r^{2(s-1)(\gamma-l-1)} \\
& \times\left.\left|\int_{\mathbb{R}}\right| \widetilde{k}^{ \pm}(\eta)\right|^{2}\left(\left|\chi(|\eta|(\alpha \mp \varphi))-\chi\left(|\eta|\left(\left(1+r^{2}\right)^{(s-1) / 2}+\alpha \mp \varphi\right)\right)\right|^{2}\right) \\
&+|\eta|^{2} r^{2 s}\left(r^{-2}\left|\chi^{\prime}(|\eta|(\alpha \mp \varphi))-\chi^{\prime}\left(|\eta|\left(\left(1+r^{2}\right)^{(s-1) / 2}+\alpha \mp \varphi\right)\right)\right|^{2}\right) \\
&+\left.r^{2(s-2) \mid}\left|\chi^{\prime}\left(|\eta|\left(\left(1+r^{2}\right)^{(s-1) / 2}+\alpha \mp \varphi\right)\right)\right|^{2} d \eta\right|^{2} d x \\
& \leq c\left\|\widetilde{K}^{ \pm} ; H^{\kappa^{2}}(\mathbb{R})\right\|^{2} \int_{\Xi_{\omega}^{ \pm}} r^{2\left(\beta^{0}-\beta^{2}-1\right)} r^{2(\gamma-l-1)} \\
& \quad \times\left(r^{2(s-1) \kappa^{2}}+r^{2 s} r^{-2} r^{-2(s-1)\left(1-\kappa^{2}\right)}+r^{2 s} r^{2(s-2)} r^{-2(s-1)\left(1-\kappa^{2}\right)}\right) d x \\
& \leq c\left\|\widetilde{K}^{ \pm} ; H^{\kappa^{2}}(\mathbb{R})\right\|^{2} \int_{R}^{\infty} r^{2\left(\beta^{0}-\beta^{2}-1\right)} r^{2(\gamma-l-1)} r^{2(s-1) \kappa^{2}} r^{s-1} r d r \\
& \leq c\left\|\widetilde{K}^{ \pm} ; H^{\kappa^{2}}(\mathbb{R})\right\|^{2} .
\end{aligned}
$$

Note that in deriving (4.70) we used the obvious inequalities

$$
\begin{gathered}
\left|\chi\left(\varrho_{1}\right)-\chi\left(\varrho_{2}\right)\right| \leq c\left|\varrho_{1}-\varrho_{2}\right|, \\
|\eta|\left|\chi^{\prime}\left(|\eta|\left(\left(1+r^{2}\right)^{(s-1) / 2}+\alpha \mp \varphi\right)\right)\right| \leq c|\eta|^{\kappa^{2}} r^{(s-1)\left(1-\kappa^{2}\right)},
\end{gathered}
$$

and the last integral in (4.70) converges due to assumption (4.63) and the definition (4.59) of the index $\kappa^{2}$.

All necessary inclusions and estimates are verified.

4.5. The proof of the main Theorem 4.1. Now everything is ready to quickly complete the proof. First, as was clarified in 3.2, the remainder $\widetilde{u}$ in the representation (4.2) satisfies the problem (1.10) with right-hand sides $\tilde{f} \in \mathfrak{V}_{\beta^{2}, \gamma}^{l-1}(G)^{k}$ and $\widetilde{g} \in \mathfrak{V}_{\beta^{2}, \gamma}^{l-1 / 2}(\partial G)^{k}$. Second, as was verified in 4.3 and 4.5 , under an appropriate choice of coefficients $c_{(q)}^{j, p}$ (see formula (4.32) and inequality (2.47) in Theorem 2.2), this remainder belongs to the space $\mathfrak{V}_{\beta^{0}-l, \gamma-l}^{1,1}(G)^{k}$. Third, Proposition 4.1 in 4.2 guarantees that $\widetilde{u} \in \mathfrak{V}_{\beta^{2}, \gamma}^{l+1,0}(G)^{k}$. Finally, the possibility to choose and admissible exponent $\beta^{0}$ in the interval $\left(\beta^{1}, \beta^{2}\right)$ is guaranteed by Remark 4.2 whereas inequality (4.3) for the norm $\left\|\widetilde{u} ; \mathfrak{V}_{\beta^{2}, \gamma}^{l+1,0}(G)^{k}\right\|$ holds because every statement that the vector-valued function $\widetilde{u}$ or some of its components belong to a certain space was accompanied by an appropriate estimate for the corresponding norms.

\section{Solvability in Weight Classes}

5.1. The weight Korn inequality. Let $\mathcal{H}$ be the Hilbert space obtained by completing the algebraic subspace $C_{c}^{\infty}(\bar{G})^{k}$ of smooth compactly supported vector-valued functions 
with respect to the norm

$$
\|u ; \mathcal{H}\|=\left(a(u, u ; G)+\left\|u ; L_{2}\left(G \cap \mathbb{B}_{2 R}\right)\right\|^{2}\right)^{1 / 2} .
$$

Following [41, by a generalized solution of the problem (1.10) we mean an element $u$ of the space $\mathcal{H}$ such that for each test function $\varphi \in \mathcal{H}$ the following integral identity is satisfied:

$$
a(u, v ; G)=(f, v)_{G}+(g, v)_{\partial G} .
$$

The proof of the existence and uniqueness of a generalized solution is usually based on information about the norm of the vector-valued function $u$ and its gradient $\nabla_{x} u$, which can be estimated from above by (5.1). To obtain this information, the weight Korn inequality can be used. In the proof of Lemma 4.2 we needed a similar inequality in the parabolic region $G_{R}^{ \pm}$; however, in 4.4 we were using in an essential way homogeneous Dirichlet conditions for the field $u$ in a noncompact portion of the boundary $\partial G_{R}^{ \pm} \backslash \Gamma_{R}^{ \pm}$. The absence of any boundary conditions makes it impossible to use known methods of proving the Korn inequality we need in this section for the general problem introduced in Section 1. We introduce the following additional condition: for each vector-valued function $u \in C_{c}^{\infty}\left(\overline{\mathbb{K}_{R}}\right)^{k}$ we have

$$
\left\|\varrho_{0} \nabla_{x} u ; L_{2}\left(\mathbb{K}_{R}\right)\right\|+\left\|\varrho_{1} u ; L_{2}\left(\mathbb{K}_{R}\right)\right\| \leq c\|u ; \mathcal{H}\| ;
$$

here $\varrho_{0}$ and $\varrho_{1}$ are the following weight functions (with some exponent $N \geq 0$ ):

$$
\varrho_{0}(x)=(1+\ln (1+r))^{-N}, \quad \varrho_{1}(x)=(1+r)^{-1}(1+\ln (1+r))^{-N-1} .
$$

We emphasize that the left-hand side of (5.4) involves only the sector $\mathbb{K}_{R}$.

Example 5.1. For a scalar operator (Example1.1 1)), this assumption holds for $N=0$ due to the one-dimensional Hardy inequality (2.12), which ensures that for $N=0$ we have

$$
\begin{aligned}
& \left\|(1+r)^{-1}(1+\ln (1+r))^{-1-N} u ; L_{2}\left(\mathbb{K}_{R}\right)\right\| \\
& \quad \leq c\left(\left\|(1+\ln (1+r))^{-N} \nabla_{x} u ; L_{2}\left(\mathbb{K}_{R}\right)\right\|+\left\|u ; L_{2}\left(\mathbb{K}_{R} \backslash \mathbb{B}_{2 R}\right)\right\|\right) .
\end{aligned}
$$

For the system of elasticity theory (Example 1.1,2)), we can take $N=1$ due to the results of 42 and the modified Hardy inequality (5.5) for $N=1$. In view of the structure of the matrix (1.8), a similar inequality holds for the system describing piezoelectric materials that was mentioned in Example 1.1, 3).

Everywhere in the rest of this section, (5.3) is assumed to be satisfied.

Lemma 5.1. For each vector-valued function $u \in \mathcal{H}$ the following weight Korn inequality is satisfied:

$$
\left\|\varrho_{0} \nabla_{x} u ; L_{2}(G)\right\|+\left\|\varrho_{1} u ; L_{2}(G)\right\| \leq c\|u ; \mathcal{H}\| .
$$

Proof. In the proof of Lemma 4.2, a similar Korn inequality was extended from the sector $\mathbb{K}_{R}$ to the entire region $G$ using the representation (4.54) for the vector-valued function $u$ and the covering of the set $G \backslash \mathbb{K}_{R}$ by trapezoids (4.53). To prove Lemma 5.1, no new ideas are needed.

Proposition 5.1. Suppose the vector-valued functions

$$
\varrho_{1}^{-1} f \in L_{2}(G)^{k}, \quad \varrho_{1}^{-1 / 2} \varrho_{0}^{-1 / 2} g \in L_{2}(\partial G)^{k}
$$


satisfy the orthogonality condition (1.32). Then the problem (1.10) has a generalized solution $u \in \mathcal{H}$. This solution is determined uniquely up to a constant summand $c \in \mathbb{C}^{k}$, and if the orthogonality condition

$$
\int_{G \cap \mathbb{B}_{2 R}} u(x) d x=0 \in \mathbb{C}^{k}
$$

holds, the solution becomes unique and satisfies the estimate $\|u ; \mathcal{H}\| \leq c \mathbf{n}$, where $\mathbf{n}$ is the sum of the norms of the vector-valued functions (5.6).

Proof. First, consider the following integral identity with parameter $\mu$ :

$$
a(u, v ; G)+\mu(u, v)_{G \cap \mathbb{B}_{R}}=(f, v)_{G}+(g, v)_{\partial G} .
$$

For $\mu>0$, the left-hand side is an inner product in the Hilbert space $\mathcal{H}$. Due to (5.6), Lemma 5.1, and a simple trace inequality

$$
\int_{\partial G} \varrho_{1} \varrho_{0}|u|^{2} d x \leq c \int_{G} \varrho_{1} \varrho_{0}\left(\left|\nabla_{x} u\right||u|+|u|^{2}\right) d x \leq c \int_{G}\left(\varrho_{1}^{2}\left|\nabla_{x} u\right|^{2}+\varrho_{0}^{2}|u|^{2}\right) d x
$$

with weight factors (5.4), the right-hand side of (5.8) is the value of a linear continuous functional on a test function $v \in \mathcal{H}$. Therefore, by the Riesz representation theorem, the integral identity (5.8) can be interpreted as an abstract equation on $\mathcal{H}$ with a selfadjoint positive definite operator. Thus, it has a unique solution $u^{\mu} \in \mathcal{H}$ for $\mu>0$. The imbedding $L_{2}(G) \subset \mathcal{H}$ is a compact operator due to the same Lemma 5.1. According to the polynomial property (see [3]), the sesquilinear form $a(u, v ; G)$ degenerates on constant elements of the space $\mathcal{H}$ only. Therefore the conclusion of Proposition 5.1 is guaranteed by the Fredholm alternative. Note that under condition (5.6) the integrals in (1.32) converge.

Corollary 5.1. Suppose inclusions (4.11) hold, $l \in \mathbb{N}$, the weight index $\gamma$ is determined by relation (1.31), and $\beta>\gamma+1$. In addition, let the right-hand sides of the problem (1.10) satisfy the orthogonality condition (1.32). Then this problem has a solution $u$ in the space $\mathfrak{V}_{\sigma, \gamma}^{l+1,0}(G)^{k}$ for $\sigma<\gamma$, which also satisfies the condition (5.7) and the estimate

$$
\left\|u ; \mathfrak{V}_{\sigma, \gamma}^{l+1,0}(G)\right\|^{2} \leq c\left(\left\|f ; \mathfrak{V}_{\beta, \gamma}^{l-1}(G)\right\|^{2}+\left\|g ; \mathfrak{V}_{\beta, \gamma}^{l-1 / 2}(\partial G)\right\|^{2}\right) .
$$

Proof. According to formulas (1.20) and (1.29), the restrictions on the weight indices $\beta$ and $\sigma$ guarantee the inclusions (5.6) and the imbedding $\mathcal{H} \subset \mathfrak{V}_{\sigma-l, \gamma-l}^{1,0}(G)^{k}$. Therefore, Lemma 5.1 yields a generalized solution $u \in \mathcal{H}$, and Proposition 4.1 increases the smoothness of this solution and puts it in the space $\mathfrak{V}_{\sigma-l, \gamma-l}^{1,0}(G)^{k}$.

5.2. "Almost energy" solutions of the problem. We assume that the orthogonality condition (1.32) does not hold and show how one can construct a solution of the problem (1.10) in this case.

For the initial terms of the formal asymptotic series constructed in 3.1 we take the solutions $U_{0}^{j, 1}$ of the homogeneous model problem (2.1) that linearly depend on $\log r$ (see formula $(2.6)$ ). Consider the sum of the first and the second terms and set

$$
\mathbf{u}(x)=\chi_{\infty}(r)\left(\sum_{j=1}^{k} b_{j} \mathcal{U}_{(0)}^{j, 1}(x)+r^{-1} \Phi_{(-1)}(\varphi, \log r)+r^{-2} \sum_{ \pm} \chi^{ \pm}(\varphi) \mathcal{V}_{(-1)}^{ \pm}\left(y^{ \pm}, \log r\right)\right),
$$

where $\mathcal{U}_{(0)}^{j, 1}$ is the asymptotic block (3.17), and $b_{1}, \ldots, b_{k}$ are some constants. The procedure for determining the corrections $r^{-1} \Phi_{(-1)}$ and $r^{-2} \mathcal{V}_{(-1)}^{ \pm}$was explained in 3.1 and 3.2. Let $\mathbf{f}$ and $\mathbf{g}$ be the mismatches determined by the vector-valued function $\mathbf{u}$ in the 
homogeneous problem (1.10). One can easily see that we have inequalities of the form (5.9) and the estimate

$$
\left\|\varrho_{1}^{-1} \mathbf{f} ; L_{2}(G)\right\|+\left\|\varrho_{1}^{-1 / 2} \varrho_{0}^{-1 / 2} \mathbf{g} ; L_{2}(\partial G)\right\| \leq c\left(\left|b_{1}\right|+\cdots+\left|b_{k}\right|\right) .
$$

Let us show that by an appropriate choice of coefficients on the right-hand side of (5.9), we can ensure that the orthogonality relations (1.32) hold for the differences $f^{\prime}=f-\mathbf{f}$ and $g^{\prime}=g-\mathbf{g}$. In this case Proposition 5.1 shows that there exists a generalized solution $u^{\prime} \in \mathcal{H}$ of the problem (1.10) with the changes $f \mapsto f^{\prime}$ and $g \mapsto g^{\prime}$. The sum $u=u^{\prime}+\mathbf{u}$ satisfies the integral identity (5.2) with any test function $v \in C_{c}^{\infty}(\bar{G})^{k}$. This sum does not belong to the class $\mathcal{H}$ because of the component (5.9) but is a classical solution of the problem with logarithmic growth at infinity. This is a small growth, so we call the resulting solution an "almost energy solution" (compare with "non-energy" solutions in the next subsection).

Let $\mathbf{R}>R$. In the Green formula for the region $G_{\mathbf{R}}^{\prime}=\{x \in G: r<\mathbf{R}\}$ we substitute the vector-valued function $\mathbf{u}$ and the constant column $e_{p}=\left(\delta_{p, 0}, \ldots, \delta_{p, k}\right)^{\top}$. We obtain

$$
\left(\mathbf{f}, e_{p}\right)_{G_{\mathbf{R}}^{\prime}}+\left(\mathbf{g}, e_{p}\right)_{\partial G_{\mathbf{R}}^{1} \cap \partial G}=\left(L \mathbf{u}, e_{p}\right)_{G_{\mathbf{R}}^{\prime}}+\left(N \mathbf{u}, e_{p}\right)_{\partial G_{\mathbf{R}}^{\prime} \cap \partial G}=-\left(N^{\mathbf{R}} \mathbf{u}, e_{p}\right)_{\partial G_{\mathbf{R}}^{\prime} \cap G}
$$

and $N^{\mathbf{R}}\left(x, \nabla_{x}\right)={\overline{\mathcal{D}\left(|x|^{-1} x\right)}}^{\top} \mathbf{A} \mathcal{D}\left(\nabla_{x}\right)$ in agreement with formulas (1.11) and (1.15). The limit as $\mathbf{R} \rightarrow+\infty$ of the left-hand side of (5.11) equals the sum $\left(\mathbf{f}, e_{p}\right)_{G}+\left(\mathbf{g}, e_{p}\right)_{\partial G}$, which is a component in the solvability conditions (1.32). We compute the limit of the right-hand side. Taking into account relations (5.9) and (3.17), as well as properties of the boundary layers $\mathcal{V}_{(-1)}^{ \pm}$, we see that the only summand that is not infinitesimally small as $\mathbf{R} \rightarrow+\infty$ is the integral

$$
\begin{aligned}
& \int_{\partial G_{\mathbf{R}}^{\prime} \cap G} \sum_{j=1}^{k} b_{j} e_{p}^{\top}{\overline{\mathcal{D}\left(|x|^{-1} x\right)}}^{\top} \mathbf{A} \mathcal{D}\left(\nabla_{x}\right) U_{(0)}^{j, 1}(x) d s_{x} \\
& =\sum_{j=1}^{k} b_{j} \int_{-\alpha / 2}^{\alpha / 2} e_{p}^{\top}{\overline{\mathcal{D}\left(|x|^{-1} x\right)}}^{\top} \mathbf{A}\left(\mathfrak{D}(\varphi, 0,1) e_{j}+\mathfrak{D}\left(\varphi, \partial_{\varphi}, 0\right) \Phi_{(0)}^{j, 1}(\varphi)\right) d \varphi+o(1) \\
& =: \sum_{j=1}^{k} M_{p j} b_{j}+o(1)
\end{aligned}
$$

Here we used formulas (2.3) $1,(2.6)$ and the fact that all the functions are smooth and the curves $\partial G_{\mathbf{R}}^{\prime} \cap G$ and $\{x: r=\mathbf{R},|\varphi|<\alpha / 2\}$ differ by circular arcs of length $O\left(\mathbf{R}^{-1}\right)$. Since, according to 3 , to the zero eigenvalue of the pencil (2.4) there correspond precisely $J$ Jordan chains of length 2 , the $k \times k$-matrix $M=\left(M_{p j}\right)$ is nonsingular (otherwise, there would be a generalized eigenvector of the second order and the length of the chain would equal at least 3; see [11, 3] and [13, Chapter 5]). Therefore, the desired property of the mismatches $\mathbf{f}$ and $\mathbf{g}$ is verified.

Note that for $\sigma<\gamma$ the vector-valued function $\mathbf{u}$ belongs to the space $\mathfrak{V}_{\sigma, \gamma}^{l+1,0}(G)^{k}$. Therefore, we have the following result.

Proposition 5.2. The conclusion of Corollary 5.1 holds even in the absence of the solvability conditions (1.32).

5.3. "Non-energy" solutions of the homogeneous problem. Computations (5.11), (5.12) almost literally repeat the procedure of [11] used to compute coefficients in the asymptotic expansion of solutions of elliptic boundaries value problems in regions with conic (corner) points. The only modification is needed to take into account the boundary layer, and it does not lead to any complications since the boundary layers decrease 
exponentially as $y_{2}^{ \pm} \rightarrow \infty$ and

$$
\left.\int_{-\alpha / 2}^{\alpha / 2} \chi^{ \pm}(\varphi) \exp \left\{-\delta y_{2}^{ \pm}\right\}\right|_{r=\mathbf{R}} d \varphi=O\left(\mathbf{R}^{-1 / 2}\right)
$$

Equally easily one can adapt the procedure from [11] to the computation of the coefficients $c_{(q)}^{j, p}$ in the expansion (4.34). Let us assume that after constructing several asymptotic terms, the asymptotic remainder, denoted now by $u$, falls into the space $\mathfrak{V}_{\sigma, \gamma}^{l+1,0}(G)^{k}$ and satisfies the problem (1.10) with the right-hand sides (4.11) and with

$$
l<\sigma<\beta .
$$

In Section 4 we established the asymptotic expansion (4.2) with the remainder $\widetilde{u}$ in the appropriate weight class and with the summands $c_{(q)}^{j, p} \mathcal{U}_{(q)}^{j, p}$ corresponding to the eigenvalues $\lambda_{q}$ between the lines $\Lambda_{l-\beta}$ and $\Lambda_{l-\sigma}$. The number $\lambda_{-q}=-\overline{\lambda_{q}}$ is also an eigenvalue for the pencil (2.4) (see 2.1). Moreover,

$$
\beta-l>\operatorname{Re} \lambda_{-q}>\sigma-l>0,
$$

so that the expression $\chi_{\infty} \mathcal{U}_{(-q)}^{h, m}$ defined according to formulas (2.5) and (3.7) by the eigenvectors and adjoint (generalized) vectors of the pencil (2.4) corresponding to $\lambda_{-q}$ does not belong to the space $\mathcal{H}$ with the norm (5.1). Using the scheme presented in 2.1 , we construct lower terms of the formal asymptotic series that follow the block $\mathcal{U}_{-q}^{h, m}$. Since each step of the iteration procedure decreases the exponent $r$ in representations (3.2) of the right-hand sides of the problem, the partial sum $\mathfrak{Z}_{(-q)}^{h, m}$ of the series can be chosen in such a way that for the vector-valued functions

$$
\mathfrak{f}_{(-q)}^{h, m}=-L \chi_{\infty} \mathfrak{Z}_{(-q)}^{h, m}, \quad \mathfrak{g}_{(-q)}^{j, p}=-N \chi_{\infty} \mathfrak{Z}_{(-q)}^{h, m}
$$

inclusions (5.6) would hold. Proposition 5.2 yields a solution $\widehat{\mathfrak{z}}_{(-q)}^{h, m} \in \mathfrak{H}$ of the problem (1.10) with right-hand sides (5.13) and a solution

$$
\mathfrak{z}_{(-q)}^{h, m}=\chi_{\infty} \mathfrak{Z}_{(-q)}^{h, m}+\widehat{\mathfrak{z}}_{(-q)}^{h, m} \in \mathfrak{V}_{2 l-\beta, \gamma}^{l+1,1}(G)^{k} \backslash \mathfrak{V}_{2 l-\sigma, \gamma}^{l+1,1}(G)^{k}
$$

of the homogeneous problem (1.10).

Due to inclusions (4.11) and (5.14) the integrals in the sum

$$
\left(f, \mathfrak{z}_{(-q)}^{h, m}\right)_{G}+\left(g, \mathfrak{z}_{(-q)}^{h, m}\right)_{\partial G}
$$

converge. An important result of [1] is that using an arbitrary polynomial-logarithmic solution $U_{(q)}^{j, p}$ of the model problem in the sector $\mathbb{K}$, we can find another polynomiallogarithmic solution $U_{(-q)}^{j, p}$ of the same problem for which

$\left.\mathbf{R} \int_{-\alpha / 2}^{\alpha / 2}\left({\overline{U_{(-q)}(x)}}^{\top} N\left(|x|^{-1} x, \nabla_{x}\right) U_{(q)}^{j, p}(x)-U_{(q)}^{j, p}(x)^{\top} \overline{N\left(|x|^{-1} x, \nabla_{x}\right) U_{(-q)}^{j, p}(x)}\right)\right|_{r=\mathbf{R}} d \varphi=1$.

Repeating, with obvious changes, computations (5.11), (5.12), one can now easily see that the coefficient $c_{(q)}^{j, p}$ in the asymptotic formula (4.2) coincides with the expression (5.15), where $h=j$ and $m=p$.

\section{Generalizations and corollaries}

6.1. The matching problem in the region with layers of constant thickness. Consider the simplest case $s=0$ in formula (1.3) where the regions $\Omega_{R}^{ \pm}$are half-strips (Figure 3). Going over Section 4 we see that all proofs hold for the matching problem (1.13) as well. The difference in asymptotic construction was already mentioned in 3.3, and the only difference in the reduction and reconstruction procedures from Section 4 
is related to the elements $\xi^{j \pm}$ and $\Xi^{j \pm}$ of the coverings. In the definition (4.5) of these elements one should replace the functions $H^{ \pm}$with the constants $-a_{ \pm}$and instead of the local estimate (4.8), use a similar local estimate of the elliptic matching problem (see [40, 43]).

6.2. The matching problem in the region with parabolic inclusions. Almost all proofs remain the same in the case $s \in(0,1)$; one should only give the correct meaning to objects on parabolic sets $\Omega_{R}^{ \pm}$(see definition (1.3) and Figure 22). The only serious modification is related to the weight Korn inequality (4.45).

According to 3.3 , an analogue of the asymptotic formula (4.2) is given by the expansions

$$
\begin{aligned}
& u(x)=\chi_{\infty}(r) \sum c_{(q)}^{j, p} U_{(q)}^{j, p}(x)+\widetilde{u}(x), \quad x \in \Omega_{R}^{0}, \\
& u(x)=\chi_{\infty}(r) \sum c_{(q)}^{j, p} U_{(q)}^{j, p}\left(r \cos \pm \frac{\alpha}{2}, \pm r \sin \pm \frac{\alpha}{2}\right)+\widetilde{u}(x), \quad x \in \Omega_{R}^{ \pm},
\end{aligned}
$$

where we use the same notation as in Theorem 4.1 .

To preserve Propositions 4.1 and 4.2, we must change the coverings of the region and extend the definition of the norms in function spaces on the parabolic set $\Omega^{1}$.

Replacing in formulas (4.5) 1 and (4.5) 2 the number $H^{ \pm}\left(y_{1}^{ \pm}\right)$with the numbers $-d<0$ and $-2 d$, respectively, we get families of rectangles $\left\{\xi^{j \pm}\right\}$ and $\left\{\Xi^{j \pm}\right\}$ covering the rays $\{x: r>2 R, \varphi= \pm \alpha / 2\}$ where we impose the matching conditions (1.13) 2 . As we have mentioned in the previous subsection, on these elements of the covering we use the local estimate of solutions of elliptic matching problems.

Coverings of the parabolic inclusions $\Omega_{R}^{ \pm}$themselves are made of rectangles $\xi_{s}^{m j \pm}$ and $\Xi_{s}^{m j \pm}$ and the following sets adjacent to the parabolic parts of the boundaries $\partial \Omega_{R}^{ \pm}$and having the structure similar to (4.48):

$$
\begin{gathered}
\xi_{s}^{j \pm}=\left\{x: 1<R^{-1} j^{1 /(s-1)} y_{1}^{ \pm}<1+\frac{j^{-1}}{1-s},-\frac{1}{4}>a_{ \pm}^{-1}\left(y_{1}^{ \pm}\right)^{-s} y_{2}^{ \pm}>-1\right\}, \\
\Xi_{s}^{j \pm}=\left\{x: 1-\frac{j^{-1}}{2(1-s)}<R^{-1} j^{1 /(s-1)} y_{1}^{ \pm}<1+\frac{2 j^{-1}}{1-s},\right. \\
\left.-\frac{1}{2}>a_{ \pm}^{-1}\left(y_{1}^{ \pm}\right)^{-s} y_{2}^{ \pm}>-1\right\} .
\end{gathered}
$$

The rectangles mentioned about fill in the space between the rows of sets (6.2) and (4.5): they are constructed in the way explained in 3.2, and as $m$ grows, their size increases proportionally to $2^{m}$. By definition, on $\Xi_{s}^{m j \pm}$ relations (4.6) hold. Since the diameter $\delta_{\Xi_{s}^{j \pm}}$ and the center of mass $x_{\Xi_{s}^{j \pm}}$ of the set $\Xi_{s}^{j \pm}$ are of order $O\left(j^{s /(1-s)}\right)$ and $O\left(j^{1 /(1-s)}\right)$, respectively, formulas (4.6) hold on the sets (6.2) as well, where, according to (1.21) we have $\rho(x)=O\left(|x|^{s}\right)$.

These properties of the coverings $\xi^{\vartheta}$ and $\Xi^{\vartheta}$ allow us to repeat the arguments in 4.2 and 4.3 without any complications. In particular, we can reduce the matching problem (1.13) to the model problem in the sector $\mathbb{K}$. In the process, we consider the spaces $\mathfrak{V}_{\beta, \gamma}^{l+1,0}\left(\Omega^{0} \cup \Omega^{1}\right)$ and $\mathfrak{V}_{\beta, \gamma}^{l-1}\left(\Omega^{0} \cup \Omega^{1}\right)$ of functions in $z$ such that their restrictions $z^{i}$ to the subregions $\Omega^{i}, i=0,1$, are in the spaces $\mathfrak{V}_{\beta, \gamma}^{l+1,0}\left(\Omega^{i}\right)$ and $\mathfrak{V}_{\beta, \gamma}^{l-1}\left(\Omega^{i}\right)$, respectively. In addition, in the first case the function of $z$ is assumed to be continuous, i.e., the first matching condition (1.13) 2 is assumed to be homogeneous. The second matching condition is allowed to be inhomogeneous, and the natural norm (1.23) is introduced in the trace space $\mathfrak{V}_{\beta, \gamma}^{l-1 / 2}\left(\partial \Omega^{1}\right)$.

The only (unfortunately, serious) obstruction to the recovery of the decay properties of asymptotic remainder arises in the proof of the weight Korn inequality (4.45). First, the 
exponent $s$ in the definition (1.2) of the region $\omega_{R}^{0}$ with artificial cutting curves must be taken the same as for parabolic inclusions (1.3). This results in a restriction to the choice of the intermediate weight exponent $\beta^{0}$ (cf. Remark 4.2 and Theorem 6.1); however, it does not affect the possibility to prove the procedure of construction the full asymptotic expansion for the solution of the problem (1.13) presented in 3.3. Second, the image of the set

$$
\left\{x: 1<R^{-1} j^{1 /(s-1)} y_{1}^{ \pm}<1+\frac{j^{-1}}{1-s},-a_{ \pm}<\left(y_{1}^{ \pm}\right)^{-s} y_{2}^{ \pm}<a_{ \pm}^{\omega}\right\},
$$

similar to (4.48), under the change $x \mapsto \eta^{j \pm}=j^{-s /(s-1)}\left(y_{1}^{ \pm}-R j^{1 /(s-1)}, y_{2}^{ \pm}\right)$of coordinates is the set

$$
\Theta_{j}^{ \pm}=\left\{x: 0<\eta_{1}^{j \pm}<\frac{R}{1-s},-a_{ \pm}\left(R+j^{-1} \eta_{1}^{j \pm}\right)^{s}<\eta_{2}^{j \pm}<a_{ \pm}^{\omega}\left(R+j^{-1} \eta_{1}^{j \pm}\right)^{s}\right\}
$$

with weakly perturbed boundary. In Section 4, perturbations affected only the part

$$
\left\{x: 0<\eta_{1}^{j \pm}<\frac{R}{1-s}, \eta_{2}^{j \pm}<a_{ \pm}^{\omega}\left(R+j^{-1} \eta_{1}^{j \pm}\right)^{s}\right\}
$$

of the boundary of the image of (4.49) where homogeneous Dirichlet conditions were imposed; through this part of the boundary, the vector-valued function $V^{j \pm}$ was extended by zero to the standard set (4.50). In the case of parabolic inclusions this method does not work, and the following assumption is necessary: for the vector-valued function $V^{j \pm} \in H^{1}\left(\Theta_{j}^{ \pm}\right)^{k}$ vanishing on the curve (6.4), the following Korn inequality, similar to (4.51), holds:

$$
\left\|V^{j \pm} ; H^{1}\left(\Theta_{j}^{ \pm}\right)\right\|^{2} \leq c\left\|\mathcal{D}\left(\nabla_{\eta^{j \pm}}\right) V^{j \pm} ; L_{2}\left(\Theta_{j}^{ \pm}\right)\right\|^{2},
$$

with a constant $c$ independent of $j \in \mathbb{N}$.

Example 6.1. Suppose a region $\Theta \subset \mathbb{R}^{2}$ with Lipschitz boundary $\partial \Theta$ has the star property with respect to the disc $\mathbb{B}_{d / 2}$. According to 42 , the Korn constant $C\left(\Theta, \mathbb{B}_{d / 2}\right)$ in the inequality

$$
\left\|V ; H^{1}(\Theta)\right\|^{2} \leq c\left(\left\|\mathcal{D}\left(\nabla_{\eta}\right) V ; L_{2}(\Theta)\right\|^{2}+\left\|V ; L_{2}\left(\mathbb{B}_{d / 2}\right)\right\|^{2}\right),
$$

where $\mathcal{D}$ is the matrix (1.7), depends on the ratio diam $\Theta / d$ only. Note that by the definition of this specific matrix $\mathcal{D}$, formula (6.6) also contains a similar Poincaré-Friedrichs inequality $\left(V\right.$ is a scalar and $\left.\mathcal{D}\left(\nabla_{\eta}\right)=\nabla_{\eta}\right)$. Since the boundary $\partial \Theta_{j}^{ \pm}$of the region (6.3) is Lipschitz, and each Lipschitz region can be represented as a union of finitely many regions with the star property with respect to a ball, the required property of the constant in (6.5) for the three problems of mathematical physics mentioned in Example 1.1 is clear.

The remaining computations in 4.4 are practically unchanged. Therefore, we established the following result.

Theorem 6.1. Let l, $\gamma, \beta^{1}$, and $\beta^{2}$ be the same as in Theorem 4.1. Additionally, suppose $\beta^{2}-\beta^{1}>s$ and the assumption about inequalities 6.5) holds. Choose $\beta^{0} \in\left(\beta^{1}, \beta^{2}\right)$ in such a way that the line $\Lambda_{l-\beta^{0}}$ does not contain points of the spectrum of the pencil (2.4) and

$$
\beta^{0}<\beta^{1}+s\left(\beta^{2}-\beta^{1}\right), \quad \beta^{0} \leq \beta^{2}-s .
$$

Then the solution $u \in \mathfrak{V}_{\beta^{1}, \gamma}^{l+1,0}\left(\Omega^{0} \cup \Omega^{1}\right)^{k}$ of the matching problem (1.13) with right-hand sides $f \in \mathfrak{V}_{\beta^{2}, \gamma}^{l-1}\left(\Omega^{0} \cup \Omega^{1}\right)^{k}$ and $g \in \mathfrak{V}_{\beta^{2}, \gamma}^{l-1 / 2}\left(\partial \Omega^{1}\right)^{k}$ admits asymptotic expansions (6.1) 


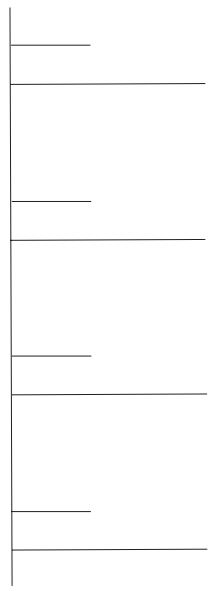

Figure 8

with the remainder $\widetilde{u}$ belonging to the space $\mathfrak{V}_{\beta^{0}, \gamma}^{l+1,0}\left(\Omega^{0} \cup \Omega^{1}\right)^{k}$; moreover, we have

$$
\begin{aligned}
& \left\|\widetilde{u} ; \mathfrak{V}_{\beta^{0}, \gamma}^{l+1,0}\left(\Omega^{0} \cup \Omega^{1}\right)\right\|+\sum\left|c_{(q)}^{j, p}\right| \\
& \quad \leq c\left(\left\|f ; \mathfrak{V}_{\beta^{2}, \gamma}^{l-1}\left(\Omega^{0} \cup \Omega^{1}\right)\right\|+\left\|g ; \mathfrak{V}_{\beta^{2}, \gamma}^{l-1 / 2}\left(\partial \Omega^{1}\right)\right\|+\left\|u ; \mathfrak{V}_{\beta^{1}, \gamma}^{l+1,0}\left(\Omega^{0} \cup \Omega^{1}\right)\right\|\right) .
\end{aligned}
$$

The sum in (6.1) and (6.7) is over those eigenvalues of the pencil (2.4) that lie between the lines $\Lambda_{l-\beta^{2}}$ and $\Lambda_{l-\beta^{0}}$.

The results presented in Section 5 can be easily adapted to the problem (1.13). We only mention that in computations (5.12) the integrals over the $\operatorname{arcs}\left\{x \in \Omega_{R}^{ \pm}: r=\mathbf{R}\right\}$ are estimated by $c \mathbf{R}^{s-1}=o(1)$.

6.3. About the boundaries of the regions and the coefficients of differential operators. In formulas (1.2)-(1.4), the boundaries of the regions are given by the formulas $y_{2}^{ \pm}=a_{0}\left(y_{1}^{ \pm}\right)^{s}$, i.e., they are arcs of parabolas. In view of the assumption (6.5), they can be perturbed:

$$
y_{2}^{ \pm}=\left(y_{1}^{ \pm}\right)^{s}\left(a_{0}+h\left(\frac{1}{y_{1}^{ \pm}}\right)\right) .
$$

Here $a_{0} \neq 0$ and, e.g., $[0,1 / R] \ni t \mapsto h(t)$ is a smooth function. Similarly one can consider the problems (1.10) and (1.13) in the case of variable coefficients by replacing conditions (1.15) and (1.16) with the stabilization conditions

$$
\begin{array}{ll}
\left|\nabla_{x}^{p}(A(x)-\mathbf{A}(\varphi))\right| \leq c_{p} r^{-\delta-p}, & x \in G_{R} \quad\left(\text { or } x \in \Omega_{R}^{0}\right), \\
\left|\nabla_{x}^{p}\left(A^{ \pm}(x)-\mathbf{A}^{ \pm}\right)\right| \leq c_{p} r^{-\delta-p}, & x \in \Omega_{R}^{ \pm}, \quad p \in \mathbb{N}_{0}, \quad \delta>0 .
\end{array}
$$

The matrix-valued function $\mathbf{A}$, which remains Hermitian and positive definite for $|\varphi| \leq \alpha / 2$, depends smoothly on the angular variable.

The requirement that the boundaries $\partial G$ and $\partial \Omega^{1}$ be smooth can be weakened by allowing either a finite number or a periodic pattern of angular points (see Figure 4). Each angular point $\mathbf{O}$ contributes powers of the weight factor $\min \{1, \operatorname{dist}(x, \mathbf{O})\}$ in the norms (1.20) and (1.29); in the second case the distribution of the powers is step-like. This class includes a well-known problem of a periodic family of boundary cracks (Figure 8).

Of course, the boundary $\partial G$ does not have to be connected (Figure 9). Outside the disc $\mathbb{B}_{R}$, the boundary $\partial \Omega$ with imposed matching conditions $(1.13)_{2}$ can be a periodic smooth or polygonal line. Disconnected sets $\partial \Omega^{0}$ for which $\Omega^{0}$ or $\Omega^{1}$ is not a region 


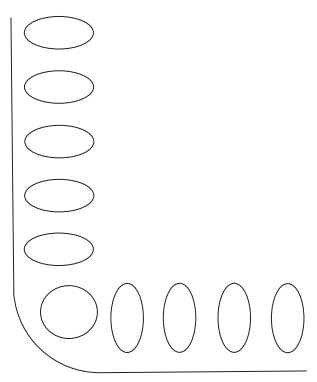

Figure 9

anymore are also allowed (see again Figure 9, in the holes the matrix $A$ takes the same value as outside the sector).

6.4. Region with parabolic inclusion. For a model problem in the case of the matching problem (1.13) in the compound region $\Omega^{0} \cup \Omega^{1}$ defined by formulas (1.2), one can take the following system of differential equations on the punctured plane:

$$
\mathbf{L}\left(\nabla_{x}\right) u(x)=f(x), \quad x \in \mathbb{K}=\mathbb{R}^{2} \backslash \mathcal{O} .
$$

The spectrum of the corresponding pencil $\mathfrak{L}\left(\varphi, \partial_{\varphi}, \lambda\right)$ is known (see, e.g., 3]). Namely, as a set, it consists of integers. Nonzero eigenvalues are algebraically simple, and their geometric multiplicity equals $k$ (the size of the system (6.8)). The complete multiplicity and all partial algebraic multiplicities of the eigenvalue $\lambda=0$ are $2 k$ and 2 , respectively. The power solutions corresponding to positive eigenvalues are polynomials. The polynomial-logarithmic solutions $U_{(0)}^{1,1}, \ldots, U_{(0)}^{k, 1}$ from the list (2.6) form a fundamental matrix $\mathcal{F}$ of the operator $\mathbf{L}\left(\nabla_{x}\right)$ on the plane, and the power solutions corresponding to negative eigenvalues are linear combinations of derivatives of the columns $\mathcal{F}^{1}=U_{(0)}^{1,1}$, $\ldots, \mathcal{F}^{k}=U_{(0)}^{k, 1}$.

6.5. The matching problem in a region with peak-like inclusion. As we have already mentioned in 1.3, the problem for a region with a parabolic inclusion discussed in the previous subsection can be transformed, using the inversion $x \mapsto \eta=|x|^{-2} x$, into the matching problem in the compound region $\omega^{0} \cup \omega^{1}$ defined by formulas (1.19) and shown in Figure 7, this region has a singular peak-like point $\mathcal{O}$.

The reduction and reconstruction procedures developed in the present paper can be applied to this problem as well, under the assumption that the constants in the Korn inequalities (6.5) for a family of regions with regularly perturbed boundaries are uniformly bounded (see Example 6.1). The norms in the weight classes $\mathfrak{V}_{\sigma, \gamma}^{l, 0}\left(\omega^{0} \cup \omega^{1}\right)$ and $\mathfrak{V}_{\sigma, \gamma}^{l}\left(\omega^{0} \cup \omega^{1}\right)$ corresponding to this problem are obtained from the norms (1.29) and (1.20) by the change of variable $r \mapsto 1 / r$. After simplifications these norms look as follows:

$$
\begin{aligned}
\left\|u ; \mathfrak{V}_{\sigma, \gamma}^{l+1,0}\left(\omega^{i}\right)\right\| & =\left(\sum_{p=0}^{l+1} \int_{\omega^{i}} r^{2\left(\sigma-\gamma-\delta_{p, 0}\right)}(r+|\varphi|)^{2\left(\gamma-l-1+\delta_{p, 0}\right)}\left|\nabla_{x}^{p} u(x)\right|^{2} d x\right)^{1 / 2}, \\
\left\|f ; \mathfrak{V}_{\sigma, \gamma}^{l-1}\left(\omega^{i}\right)\right\| & =\left(\sum_{p=0}^{l-1} \int_{\omega^{i}} r^{2(\sigma-\gamma)}(r+|\varphi|)^{2(\gamma-l+1)}\left|\nabla_{x}^{p} f(x)\right|^{2} d x\right)^{1 / 2} .
\end{aligned}
$$

Here $\varphi \in[-\pi, \pi]$, and the polar axis is directed inside the peak, i.e., $\varphi= \pm \pi$ on the extension of the peak. 


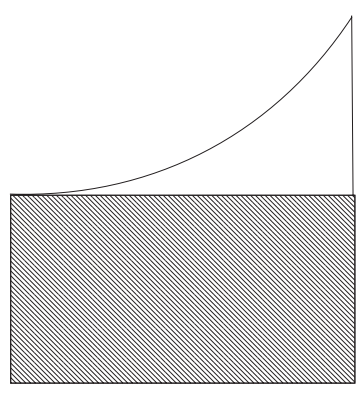

FiguRE 10

It is clear that under inversion the operator $L\left(x, \nabla_{x}\right)$ loses many required properties, such as, e.g., polynomiality [3] and the algebraic completeness [1] of the matrix $\mathcal{D}$. Therefore, a direct reference to Theorem 6.1 is not sufficient to obtain asymptotic formulas, and one must repeat (almost literally) the arguments and computations that are presented in this paper for the problem with a region going to infinity. Instead of doing this, we mention once again the papers [23, 24] where the formal asymptotic analysis was carried out for the equations of elasticity theory. We emphasize that the relation between the problems for the compound regions $\Omega^{0} \cup \Omega^{1}$ and $\omega^{0} \cup \omega^{1}$ established by inversion gives correct transformations of geometric objects, i.e., of coverings $\left\{\xi^{Q}\right\},\left\{\Xi^{Q}\right\}$, of variability zones for the cut-off functions, etc.

The problem for an angular elastic body with a thinning elastic inclusion (Figure 10) can also be analyzed using this approach.

\section{REFERENCES}

[1] Nečas J. Les méthodes directes en théorie des équations elliptiques. Masson, Paris; Prague, 1967. MR0227584(37:3168)

[2] S. A. Nazarov, Self-adjoint elliptic boundary. Polynomial property and formally positive operators. Problemy Mat. Analiza, no. 16, SPb. Univ., St-Petersburg, 1997, pp. 167-192; English transl., J. Math. Sci. (New York) 92 (1998), no. 6, 4338-4353. MR1668363 (99h:00043) MR1668418 (2000a:35042)

[3] , Polynomial property of selfadjoint elliptic boundary value problems, and the algebraic description of their attributes. Uspekhi Mat. Nauk 54 (1999), no. 5(329), 77-142; English transl., Russian Math. Surveys 54 (1999), no. 5, 947-1014. MR1741662 (2001k:35073)

[4] E. Sanchez-Palencia and P. Suquet, Friction and homogenization of a boundary. Free Boundary Problems: Theory and Applications. A. Fasano, M. Primicerio (eds.). Pitman, London, 1983, pp. 561-571. MR714936 (86b:73036)

[5] M. Lobo and M. Pérez, Local problems for vibrating systems with concentrated masses: a review. C. R. Mecanique 331 (2003), 303-317.

[6] S. A. Nazarov, Binomial asymptotic behavior of solutions of spectral problems with singular perturbations. Mat. Sb. 181 (1990), no. 3, 291-320; English transl., Math. USSR-Sb. 69 (1991), no. 2, 307-340. MR1049991 (91d:35160)

[7] Y. Amirat, G. A. Chechkin, and R. R. Gadyl'shin, Asymptotics of simple eigenvalues and eigenfunctions for the Laplace operator in a domain with oscillating boundary, J. Comp. Math. Math. Phys. 46 (2006), no. 1, 102-115. MR2239730(2007d:35009)

[8] A. M. Il'in, Matching of asymptotic expansions of solutions of boundary value problems. "Nauka", Moscow, 1989; English transl., Amer. Math. Soc., Providence, RI, 1992. MR1182791 (93g:35016)

[9] W. G. Mazja, S. A. Nazarov, and B. A. Plamenewski, Asymptotische Theorie elliptischer Randwertaufgaben in singulär gestörten Gebieten. 1. Akademie-Verlag, Berlin, 1991; English transl., V. Maz'ya, S. Nazarov, and B. Plamenevskij, Asymptotic theory of elliptic boundary value problems in singularly perturbed domains. Vol. 1, Birkhäuser, Basel, 2000. MR.1101139 (92g:35059)

[10] V. A. Kondrat'ev, Boundary value problems for elliptic equations in domains with conical or angular points. Trudy Moskov. Mat. Obshch. 16 (1967), 209-292. (Russian) MR0226187 (37:1777) 
[11] V. G. Maz'ja and B. A. Plamenevskiı̌, The coefficients in the asymptotics of solutions of elliptic boundary value problems with conical points. Math. Nachr. 76 (1977), 29-60. (Russian) MR0601608 $(58: 29176)$

[12] - The coefficients in the asymptotic expansion of the solutions of elliptic boundary value problems near an edge. Dokl. Akad. Nauk SSSR 229 (1976), no. 1, 33-36. (Russian) MR0407446 $(53: 11221)$

[13] S. A. Nazarov and B. A. Plamenevsky, Elliptic problems in domains with piecewise smooth boundaries. Walter de Gruyter, Berlin-New York, 1994. MR1283387 (95h:35001)

[14] V. A. Kozlov, V. G. Maz'ya, and J. Rossmann, Elliptic boundary value problems in domains with point singularities. Amer. Math. Soc., Providence, RI, 1997. MR.1469972 (98f:35038)

[15] S. A. Nazarov, Asymptotic behavior of the solution of the Dirichlet problem in an angular domain with a periodically changing boundary. Mat. Zametki 49 (1991), no. 5, 86-96; English transl., Math. Notes 49 (1991), no. 5-6, 502-509. MR.1137177 (92g:35062)

[16] _ The Dirichlet problem for an elliptic system with periodic coefficients in a corner domain. Vestnik Leningrad. Univ. Mat. Mekh. Astronom. 1990, vyp. 1, 32-35; English transl., Vestnik Leningrad Univ. Math. 23 (1990), no. 1, 33-35. MR1098480 (91m:35079)

[17] _ Asymptotic expansion of the solution of the Dirichlet problem for an equation with rapidly oscillating coefficients in a rectangle. Mat. Sb. 182 (1991), no. 5, 692-722; English transl., Math. USSR-Sb. 73 (1992), no. 1, 79-110. MR1124104 (92h:35038)

[18] - Asymptotics at infinity of the solution to the Dirichlet problem for a system of equations with periodic coefficients in an angular domain. Russian J. Math. Phys. 3 (1995), no. 3. 297-326. MR:1370627 (97g:35034)

[19] S. A. Nazarov and A. S. Slutskiı̌, Asymptotic behavior of solutions of boundary value problems for an equation with rapidly oscillating coefficients in a domain with a small cavity. Mat. Sb. 189 (1998), no. 9, 107-142; English transl., Sb. Math. 189 (1998), no. 9-10, 1385-1422. MR1680848 (2000a:35014)

[20] F. Blanc and S. A. Nazarov, Asymptotics of solutions to the Poisson problem in a perforated domain with corners. J. Math. Pures. Appl. 76 (1997), no. 10, 893-911. MR1489944 (98h:35017)

[21] G. Caloz, M. Costabel, M. Dauge, and G. Vial, Asymptotic expansion of the solution of an interface problem in a polygonal domain with thin layer. Asymptotic Analysis. 50 (2006), no. 1, 121-173. MR2286939

[22] D. Leguillon and E. Sanchez-Palencia, Computation of singular solutions in elliptic problems and elasticity. Masson, Paris; Wiley, New York, 1987. MR995254 (90m:73015)

[23] A. B. Movchan and S. A. Nazarov, Asymptotic behavior of the stress-strained state near sharp inclusions. Dokl. Akad. Nauk SSSR 290 (1986), no. 1, 48-51; English transl., Soviet Phys. Dokl. 31 (1986), 772-774. MR857872 (88c:73019)

[24] _ Stress-deformed states in the vertex of a sharp inclusion. Mekh. Tverdogo Tela, 1986, no. 3, 155-163. (Russian)

[25] _ Asymptotics of a stress-deformed state near a spatial pique-like inclusion. Mekh. Kompoz. Materialov, 1985, no. 5, pp. 792-800. (Russian)

[26] _ Asymptotics of the solution to the Neumann problem in a domain with singular point of peak exterior type. Russian J. Math. Phys. 4 (1996), no. 2, 217-250. MR1414884 (2000d:35044)

[27] V. G. Maz'ya, S. A. Nazarov, and B. A. Plamenevskiı̌, Elliptic boundary value problems in domains of the type of the exterior of a cusp. Linear and nonlinear partial differential equations. Spectral asymptotic behavior, pp. 105-148, Probl. Mat. Anal., 9, Leningrad. Univ., Leningrad, 1984. (Russian) MR.772047 (86g:35063)

[28] S. A. Nazarov, Estimates near an edge for the solution of the Neumann problem for an elliptic system. Vestnik Leningrad. Univ. Mat. Mekh. Astronom. 1988, no. 1, 37-42; English transl., Vestnik Leningrad Univ. Math. 21 (1988), no. 1, 52-59. MR946462 (89h:35108)

[29] S. A. Nazarov and B. A. Plamenevskiŭ, The Neumann problem for selfadjoint elliptic systems in a domain with a piecewise-smooth boundary. Trudy Leningrad. Mat. Obshch., Vol. 1, 1990, pp. 174211. (Russian) MR:1104210 (92e:35065)

[30] M. S. Agranovich and M. I. Vishik, Elliptic problems with a parameter and parabolic problems of general type. Uspehi Mat. Nauk 19 (1964), no. 3, 53-161; English transl., Russian Math. Surveys 19 (1964), no. 3, 53-157. MR0192188(33:415)

[31] V. G. Maz'ja and B. A. Plamenevskiı̌, Weighted spaces with inhomogeneous norms, and boundary value problems in domains with conical points. Elliptische Differentialgleichungen (Rostock, 1977), pp. 161-190, Wilhelm-Pieck-Univ., Rostock, 1978. (Russian) MR.540196 (81e:35045) 
[32] Elliptic boundary value problems on manifolds with singularities. Problems in mathematical analysis, No. 6: Spectral theory, boundary value problems, pp. 85-142, Izdat. Leningrad. Univ., Leningrad, 1977. (Russian) MR0509430 (58:23025)

[33] S. A. Nazarov, Asymptotic behavior with respect to a parameter of the solution to a boundary value problem that is elliptic in the sense of Agranovich-Vishik in a domain with conical points. Boundary value problems. Spectral theory, pp. 146-167, 243-244, Probl. Mat. Anal., 7, Leningrad. Univ., Leningrad, 1979. (Russian) MR.559107 (81a:35030)

[34] V. A. Nikishkin, Singularities of the solution of the Dirichlet problem for a second-order equation in the neighborhood of an edge. Vestnik Moskov. Univ. Ser. I, Mat. Mekh. no. 2 (1979), 51-62. (Russian) MR:531648 (80h:35046)

[35] V. G. Maz'ya and J. Rossmann, Uber die Asymptotik der Lösungen elliptischer Randwertaufgaben in der Umgebung von Kanten. Math. Nachr. 138 (1988), 27-53. MR975198 (90a:35079)

[36] S. A. Nazarov, Nonselfadjoint elliptic problems with the polynomial property in domains possessing cylindrical outlets to infinity. Zap. Nauchn. Sem. St.-Peterburg. Otdel. Mat. Inst. Steklov. (POMI) 249 (1997), 212-230; English transl., J. Math. Sci. (New York) 101 (2000), no. 5, 3512-3522. MR 1698519 (2001b:35087)

[37] _ The Vishik-Lyusternik method for elliptic boundary value problems in regions with conic points. II. Problem in a bounded domain. Sibirsk. Mat. Zh. 22 (1981), no. 5, 132-152; English transl., Siberian Math. J. 22 (1981), 753-769. MR632823 (83m:35046b)

[38] S. Agmon, A. Douglis, and L. Nirenberg, Estimates near the boundary for solutions of elliptic differential equations satisfying general boundary conditions. 2. Comm. Pure Appl. Math. 17 (1964), 35-92. MR.0162050 (28:5252)

[39] V. A. Solonnikov, General boundary value problems for systems elliptic in the sense of A. Douglis and L. Nirenberg. I. Izv. Akad. Nauk SSSR Ser. Mat. 28 (1964), 665-706; II. Trudy Mat. Inst. Steklov. 92 MR0211070 (35:1952)

[40] J.-L. Lions and E. Magenes, Problèmes aux limites non homogènes et applications. Vol. 1. Dunod, Paris, 1968. MR0247243(40:512)

[41] O. A. Ladyzhenskaya, The boundary value problems of mathematical physics. Springer-Verlag, New York, 1985. MR793735 (87f:35001)

[42] V. A. Kondrat'ev and O. A. Oleinik, Boundary value problems for a system of elasticity theory in unbounded domains. Korn inequalities. Uspekhi Mat. Nauk 43 (1988), no. 5(263), 55-98; English transl., Russian Math. Surveys 43 (1988), no. 5, 65-119. MR971465 (89m:35061)

[43] Ya. A. Roitberg and Z. G. Sheftel, General boundary-value problems for elliptic equations with discontinuous coefficients. Dokl. Akad. Nauk SSSR 148 (1963), 1034-1037. (Russian) MR0146507 $(26: 4029)$

St. Petersburg Branch, Institute of Machine Behavior, Russian Academy of Sciences, St. Petersburg, Russia

E-mail address: serna@snark.ipme.ru 